\title{
Illusory contours: Toward a neurally based perceptual theory
}

\author{
GREGORY W. LESHER \\ Enkidu Research, Rochester, New York
}

\begin{abstract}
Although illusory contours were first described nearly a century ago, researchers have only recently begun to approach a consensus on the processes underlying their formation. Neurophysiological and psychophysical evidence indicate that neural mechanisms of the early visual cortex subserve illusory contour generation, although cognitive factors play important roles in determining the final percept. I summarize experiments concerning the determinants of illusory contour strength and form, concentrating on findings particularly relevant to modeling. After establishing arguments for the early generation of illusory contours, I provide an overview of formation theories, culminating with descriptions of neural models. The constraints that experimental data place on models are outlined, and neural models are evaluated with respect to these constraints. Throughout the review, I indicate where further experimental and modeling research are critical.
\end{abstract}

Schumann introduced the first illusory contour, depicted in Figure 1a, in 1900, noting that a central "white rectangle with sharply defined contours appears, which objectively are not there." Schumann had discovered and noted two salient features of illusory figures: sharp edges in regions of homogeneous luminance, and a brightening within the figure. Perhaps because this stimulus failed to yield particularly convincing illusory contours, it was not until more than 50 years later that illusory contours became a topic for active research. Although Ehrenstein (1941) demonstrated stimuli with salient illusory contours, it is remarkable that he failed to note their existence in the text of his article explicitly, commenting instead only on illusory brightening effects. Kanizsa's (1955) stunning figures, examples of which are depicted in Figures $1 b$ and $1 \mathrm{c}$, produced extremely sharp, salient contours along with obvious brightening, initiating a wave of research.

Although there have been a number of excellent illusory contour review papers (Halpern \& Salzman, 1983; Meyer \& Petry, 1987; Parks, 1984), research advances in the psychophysical, neurophysiological, and modeling domains have been more than sufficient to mandate a new summary of the literature. In this review, I will not only present more recent data, but also employ an approach to the summaries that is markedly different from those previously taken. At the time of the earlier reviews, determination of the locus of formation of illusory contours was the central issue in illusory contour research, a fact reflected in the structure of the review papers. Experimental results were presented primarily in the historical context of supporting or refut-

I would like to thank Ennio Mingolla of Boston University for generously providing many helpful comments and suggestions during the development of this review. Correspondence should be addressed to $G$. W. Lesher, Enkidu Research, 2250 N. Triphammer Road, \#N3A, Ithaca, NY 14850 (e-mail: lesher@cns.bu.edu). ing particular theories of contour formation (although Parks's "(Mostly) Atheoretical Review" is something of an exception to this rule). Within the last half decade, however, as neural models of illusory contour formation have become predominant, most of these earlier theories have fallen out of favor-although no single neural model has received universal acceptance. Strong neurophysiological evidence, combined with a body of convincing psychophysical data, has caused strictly retinal and strictly cognitive factors to be less important to the attempt to explain contour formation. Although cognitive factors can play a profound role in determining the salience of illusory figures, it appears that low- to intermediate-level mechanisms are both necessary and sufficient to establish the perceptual foundation from which the bulk of both psychophysical and neurophysiological data can be adequately explained.

Most neural models of contour formation are still in their infancy, lacking the sophistication needed to model any but the simplest of illusory phenomena. Even more mature models, such as the boundary contour system (Grossberg \& Mingolla, 1985a, 1985b, 1987a, 1987b), have yet to demonstrate, through simulations, all the effects that they are potentially capable of emulating. However, it is to be expected that the additional focus on neural models will greatly accelerate the pace of their development. Therefore, a comprehensive review of the psychophysical and neurophysiological data relevant to such modeling would be of significant value. The purpose of this review is twofold: first, to establish a checklist and summary of the determinants of illusory contour strength, and second, to review and critically examine the various neural models of illusory contour formation. Rather than review the entire body of illusory contour literature-in their bibliographical review, Purghé and Coren (1992b) have cited some 445 references - I concentrate on summarizing and analyzing the experimental findings that one can reasonably 
a

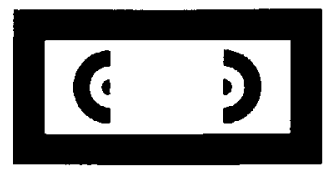

b

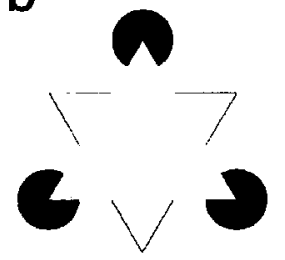

C

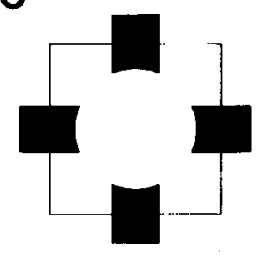

Figure 1. (a) Schumann's (1900) original illusory figure; (b) Kanizsa's (1955) triangle; and (c) example of curved illusory contours. Figures $1 \mathrm{~b}-1 \mathrm{c}$ are from "Margini Quasi-Percettiví in Campi con Stimulazione Omogenea," by G. Kanisza, 1995, Rivista di Psicologia, 49, pp. 7-30 (Figures 10-11). Copyright 1955 by Giunti Gruppo Editoriale. Reprinted with permission.

expect to be modeled within the next decade. In areas where our current knowledge is incomplete, the research and experiments necessary to fill the gaps are indicated. The general complexity of neural models of contour formation precludes complete computational specification within this review. I therefore provide only brief summaries of the models, with a concentration on the motivation and unique characteristics of each model. Although the review is structured with the the neural or computational modeler in mind, I have endeavored also to make it informative for empirical and theoretical researchers.

The remainder of the review is partitioned into seven sections, as outlined below. In the section on Types of Illusory Contours, I present a definition of illusory contours and analyze the different stimuli that generate these contours, with emphasis on monocular stimuli defined solely in the luminance domain. The three major perceptual characteristics of illusory contours and figures - strength, brightness, and depth - are examined in Characteristics of Illusory Figures. In the next section, Determinants of Illusory Contour Strength, I introduce and summarize the various factors that affect the strength of illusory contours and the degree of illusory brightening. Neurophysiological and psychophysical evidence that illusory contours are instantiated early in the visual processing stream are presented in Illusory Contours as Early Vision Phenomena. In Phenomenological Models of Illusory Contour Formation, a history of various theories of contour generation is tendered, during the course of which additional psychophysical data are presented and a basis for subsequent neural models is established. These computational models are reviewed in Neural Models of Illusory Contour Formation, with the subsequent section, Developing and Eval- uating Neural Models, containing an analysis of the constraints that the psychophysical data place on neural models, as well as an evaluation of the degree to which the current models satisfy these constraints. Finally, in the Summary, I present an overview of the current state of research and modeling.

\section{TYPES OF ILLUSORY CONTOURS}

An illusory contour is defined as the percept of a clear boundary in regions where there is no corresponding luminance gradient, as in the stimuli of Figures 1 and 2. Illusory contours are modal in nature (Kanizsa, 1955, 1976) they are perceptually salient, appear to belong to the figure rather than the ground, and have a real phenomenological presence. This is an important, if somewhat ambiguous, characteristic. Immediately after presentation of Figure 1a, Schumann $(1900,1904)$ goes on to observe a "subjective contour" connecting the line segments of Figure 3a. Kanizsa refers to this percept and to the lines or arcs connecting the dots of Figure $3 \mathrm{~b}$ as "virtual lines." He notes that while virtual lines have a definite perceptual presence, such lines are not nearly as salient, as real, as illusory contours - they are amodal in character. While the distinction is perhaps one of degrees, vision researchers today would generally not refer to the groupings of Figure 3 as illusory contours.

The term "boundary" was employed in the definition of illusory contours to avoid the loaded connotation of luminance difference associated with the term "edge." In all of the stimuli of Figures 1 and 2, the illusory contours are boundaries between two perceptual regions. Specifically, in all but Figure 2c, the illusory contour serves as a boundary a
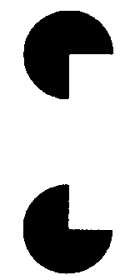

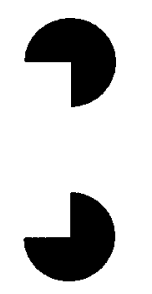

b

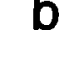

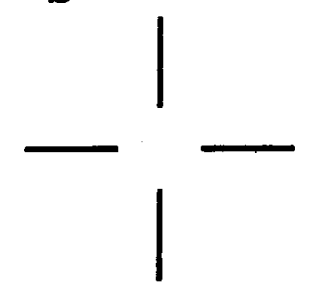

C

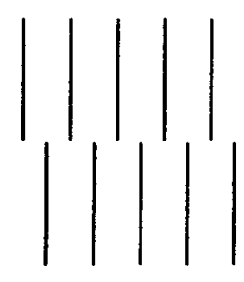

Figure 2. (a) Edge-induced illusory contour; (b) line-end induced illusory contour; (c) line-end induced illusory contour without illusory figure. 
a

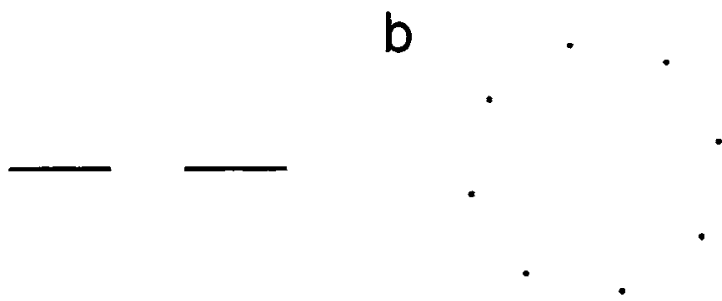

Figure 3. (a) The other "subjective contour" of Schumann (1900); (b) a series of dots, like Schumann's lines, give rise to a "virtual line" (circle) that does not have the perceptual salience of an illusory contour:

between a partially specified figure and the inducing elements that it occludes. In these cases the illusory figure is modally observed, with a clear brightness difference between the figure and the identically contrasted background. In the dark-on-light examples provided here, there is an illusory brightening, but for light inducers on a dark background an illusory darkening is observed (Ehrenstein, 1941; Kanizsa, 1955). In Figure 2c, there is no illusory figure and no consistent brightening or darkening - although there may be some local brightness effects near the ends of the individual lines-but the illusory contour still marks a perceptual boundary between the two regions.

The definition of illusory contours and figures refers only to their phenomenological appearance, with no reference to the underlying stimuli responsible for generating the contours. Therefore illusory contours are not bound to any one class of inducing stimuli, but may in fact be generated by a range of different stimuli configurations in the luminance, stereo, and kinetic domains. I will be primarily concerned with stimuli defined solely in the luminance domain, such as those in Figures 1 and 2, providing only brief overviews of illusory contours generated in the other domains. There are relatively few studies of intermodally generated illusory contours, but current research appears to indicate a gratifying symmetry between domains - inducers and the resulting illusory contours appear to interact in similar ways regardless of the inducer modalities (Kellman \& Loukides, 1987; Prazdny, 1986). These intermodal similarities, the relative immaturity of illusory contour research in nonluminance domains, the breadth of research in the luminance domain, and the need to limit this paper to a manageable scope warrant the concentration on luminance-defined contours. Nevertheless, a basic understanding of how illusory contours defined in nonluminance domains are related to the more familiar luminance-defined contours will be essential to future modeling efforts.

The ecological significance of illusory figures is clear. Creatures with the ability to perceive illusory forms, to disambiguate figure from ground under conditions of near equiluminance and equicolor, are provided with a strong advantage over creatures that lack this ability. Illusory contours represent a powerful method for defeating camouflage of both predator and prey (Ramachandran, 1986a, 1987). They doubtless also play important roles in navigation, providing a more accurate model of the external world. The benefits of illusory contour perception are es- pecially apparent under scotopic conditions, where figureground contrasts are often insignificant. As Rock and Anson (1979) have stated, illusory figures are the solutions to complex perceptual problems: What are the most probable organizations that account for the stimuli? The ecological relevance of illusory contours renders it highly unlikely that they simply represent epiphenomena of other visual processes. Rock and Anson defined their ecological problem in the context of a cognitive approach to illusory contour formation, but there is no inherent link between the perceptual challenge and any one method of arriving at the answer. We can, for the time being, atheoretically pursue the significance of illusory forms.

\section{Edge-Induced Illusory Contours}

The class of illusory contours defined solely by luminance variations can be further divided into two subclasses. Whether or not these two classes indicate distinct underlying mechanisms for contour completion will be discussed later in the review. The first of these types, typified by Figures $1 \mathrm{a}$ and $1 \mathrm{~b}$ and Figure 2a, consist of solid inducing elements containing edges or gaps locally consistent with a noncoincidental occluding figure of the same luminance as that of the background. That is, without needing to postulate a coincidental layout of the occluding figure(s), one can easily imagine that each gap arises from a simple occlusion. The illusory contour is collinear to those edges of the inducers that are consistent with the aforementioned occlusion, and it persists in regions of homogeneous luminance between edges of adjacent inducers. Since completion is in directions roughly collinear to the inducer edges, these types of inducers are referred to as edge inducers. The inducer edges that specify the illusory figure by real luminance gradients are referred to by Lesher and Mingolla (1993) as "supporting edges," while the portions of the illusory figure adjacent to supporting edges are referred to as "supported." In Figure 2a, for example, the illusory figure is supported within the missing sector of each circular inducer. The illusory contour itself is by definition unsupported.

The constraint that edge-inducing stimuli be locally consistent with occlusion dovetails nicely with the ecological motivation for illusory forms, but it should not be taken as implying that any such occlusion need be perceived for illusory contour formation to occur. The consistency constraint is simply a convenient and intuitive way to relate a characteristic shared by all edge inducers. Shipley and Kellman (1990) may have succeeded in defining local consistency in more concrete, geometric terms by showing that discontinuities (corners and line ends) in the inducer structure are both strong indications of occlusion and strong determinants of illusory contour formation. As Figure $4 \mathrm{a}$ indicates, supporting edges may be on the inside of the illusory form, indicating local self-occlusion of markings on the figure surface by the edges of the figure. Local consistency with occlusion implies that not all supporting edges need be consistent with a single coherent occluding form, as is evidenced by the examples of Figure $4 \mathrm{~b}$, which have illusory contours supported on both the inside and 
a

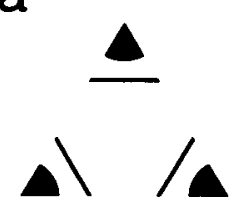

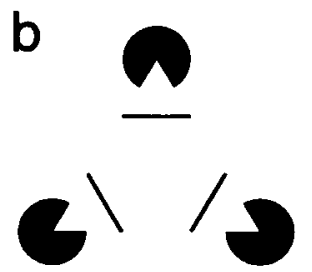

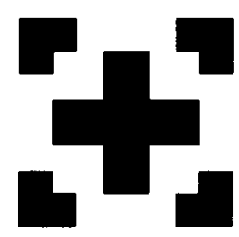

Figure 4. (a) Inducers on the inside of the illusory figure can also drive illusory contour formation (Kennedy \& Lee, 1976). (b) Combinations of inducers on the inside and outside of the figure indicate that there need be no global consistency with occlusion. Figures $4 a$ and $4 b$ (left) are from "A Figure-Density Hypothesis and Illusory Contour Brightness," by J. M. Kennedy and H. Lee, 1976, Perception, 5, pp. 387-392 (Figures 4-5). Copyright 1995 by Pion Limited, London. Adapted with permission. Figure 4b (right) is from "On the Filtered-Components Approach to Illusory Visual Contours," by T. E. Parks and L. Pendergrass, 1982, Perception \& Psychophysics, 32, pp. 491-493 (Figure 2). Copyright 1982 by Psychonomic Society, Inc. Adapted with permission.

the outside of the resulting illusory figure (Kennedy, 1979; Kennedy \& Lee, 1976; Parks, 1986; Parks \& Pendergrass, 1982). In all the stimuli of Figure 4 , the supporting regions are also locally consistent with a configuration in which the illusory contour represents a hole in the foreground, through which partially occluded inducers are visible, even though this may not necessarily be the percept.

While local consistency with occlusion marks a general constraint on the existence of illusory contours, there are more specific characteristics that profoundly influence illusory contour formation. Most importantly, the shape of the illusory figure typically must be well specified by the inducers - points of high curvature must fall within the part of the figure supported by inducer edges. This implies that multiple supporting edges are necessary, and that adjacent supporting edges must be at least partially aligned with one another. Additional factors that affect the formation of illusory contours and their resulting strengths include the degree of alignment of supporting edges, number of inducers, degree of support, inducer contrast, and inducer shape. In addition, such cognitive determinants as perceptual set and memory may influence contour formation. These, and other, factors will be discussed in detail later in the review.

\section{Line-End Induced Illusory Contours}

As the region of support becomes smaller in relation to the illusory contour length, the inducing elements progress from edge inducers to line-end inducers, examples of which are depicted in Figures $2 b$ and $2 c$. Line-end inducers must fulfill the same basic criteria as edge inducers regarding sufficient specification of the illusory figure shape, but the resulting illusory contours are not collinear with the regions of support, but rather in directions roughly perpendicular to the supporting lines. Whereas with edge inducers the illusory figure is tightly constrained in both position and orientation within supported regions, in line-end illusory contours the inducers primarily constrain position-orientation tends to be approximately perpendicular to the inducing lines, but it may vary significantly from this angle (Gillam, 1987; Gillam \& Goodenough, 1994; Parks, 1980). Most of the factors that influence the perception of edge-induced illusory contours also play roles in determining the strength of contours induced by line ends.

Illusory contours may persist if colored line segments are added which span the linear or curvilinear gaps between corresponding line ends (e.g., a colored cross inserted in the center of an Ehrenstein figure) (van Tuijl, 1975; van Tuijl \& de Weert, 1979; Varin, 1971). In such stimuli, the line color may spread to fill the illusory figure--a phenomenon known as neon color spreading. There is a correlation between the degree of neon color spreading and the strength of the bounding illusory contour (Watanabe \& Takeichi, 1990), although the degree of neon color spreading is also dependent on stimulus characteristics unrelated to illusory contour formation, such as the degree of alignment between inducing lines and colored segments (Redies, 1989; Redies \& Spillman, 1981; Redies, Spillman, \& Kunz, 1984; Watanabe \& Sato, 1989).

There is no clear classification boundary between edge and line-end induced contours. A line always has some width and therefore provides edge information, albeit at a very small scale. Conversely, an edge inducer has two corners, the nonsupporting edges of which are often roughly perpendicular to the supporting edges, and thus provides something akin to line-end information. Gradually increasing the width of a line results in a smooth progression from line end to edge inducer, with clear perception of an illusory contour throughout the entire transition (Lesher \& Mingolla, 1993; Petry, Harbeck, Conway, \& Levey, 1983). The role of edges and line ends in illusory contour formation poses one of the fundamental questions in modeling these phenomena: Do the two types of inducers imply the existence of two completely separate mechanisms or extremes of the same mechanism? Alternatively, we might ask: Do both parallel and perpendicular completion occur during illusory contour formation?

\section{Other Types of Illusory Contours}

The definition of illusory contours provided above-a clear boundary in a region of homogeneous luminancecaptures several phenomena not traditionally considered illusory contours. In particular, illusory contours induced 
in the stereo and kinetic domains have not typically been recognized as such. It has long been known that the strength of illusory contours induced by both edge and line-end stimuli can be enhanced by providing additional stereo or motion cues, such as to increase the salience of the perceptual boundary between illusory figure and inducing elements (Gregory \& Harris, 1974; Lawson, Cowan, Gibbs, \& Whitmore, 1974; Prazdny, 1985; Ramachandran, 1986a, 1987; Ramachandran \& Cavanagh, 1985; Whitmore, Lawson, \& Kozora, 1976). In these examples, contours were already established in the monocular, luminance domainstereo and kinetic cues only mediated their strengths. However, illusory contours can be induced without any supporting luminance discontinuities whatsoever.

In the stereo domain, Julesz's (1960) random dot stereograms give rise to strong illusory contours at the edges of the disparate regions, although they were not recognized as such until much later (Julesz \& Frisby, 1975). In these cases, illusory contours are observed only in regions where there are local depth disparities (i.e., areas proximal to elements with definite disparity), but Prazdny (1986) and Mustillo and Fox (1986) have reported illusory contour formation in random dot stimuli in which only the edge inducers of a Kanizsa figure are defined by local disparity. Contour completion occurs between these stereoscopically defined inducers across areas with no local luminance or depth discontinuities-a remarkable fact when one considers that in the completed regions there is substantial evidence, in the form of dot pairs specifying depth behind the inducing elements, against the existence of an occluding illusory figure. Similarly, in the kinetic domain, illusory contours can be specified by local motion cues (Andersen \& Cortese, 1989; Bruno \& Bertamini, 1990; Bruno \& Gerbino, 1991; Kaplan, 1969; Kellman \& Loukides, 1987; Shipley \& Kellman, 1994; Stappers, 1989) or can be induced with no local cues, but with motion-defined inducers (Kellman \& Loukides, 1987; Prazdny, 1986). Cases of illusory contour generation in the absence of local stereo or kinetic cues are examples of second-order contour completion. Local cues give rise to inducers, themselves illusory, which then behave analogously to luminance-defined inducers, resulting in illusory contour completion across regions with no local stereo or kinetic cues. There have been no experiments to determine whether such second-order inducers behave equivalently to luminance-defined inducers under parametric variations of inducer characteristics (e.g., inducer size and distance between inducers).

In addition to contours defined solely in the stereo or kinetic domains, several classes of illusory contours must be defined in both the luminance and the motion domains. These include moving phantoms (Maguire \& Brown, 1987; Mulvanny, Macarthur, \& Sekuler, 1982; Tynan \& Sekuler, 1978; Weisstein, Maguire, \& Berbaum, 1977), stereokinetic contours (Bressan \& Vallortigara, 1986, 1987, 1991; Vallortigara, 1987; Vallortigara, Bressan, \& Zanforlin, 1986; Zanforlin \& Vallortigara, 1990), and rotationinduced contours (Klymenko \& Weisstein, 1981, 1983, 1984, 1987; Klymenko, Weisstein, \& Ralston, 1987). All these stimuli are similar in that the potential for comple- tion exists when the inducers are static-they have aspects of edge or line-end stimuli-but not until the inducers move does the illusory contour become salient. Note that an experienced observer may report the illusory contours even without motion. A number of researchers (Kanizsa, 1979; Petter, 1956) have noted a related class of luminance-defined stimuli, referred to as "spontaneously splitting figures" by Kellman and Shipley (1991), that have multiple potentials for completion that are only realized under certain perceptual (as opposed to stereo or kinetic) conditions - the perceived depth ordering of the figures determines the contour visibilities.

An understanding of illusory contours defined in nonluminance domains is essential to the development of a global model of illusory contour formation and of early vision in general. Using a tilt aftereffect paradigm, Paradiso, Shimojo, and Nakayama (1989) showed that there is some cross-adaptation between real and illusory contours. Berkley, Debruyn, and Orban (1994) have significantly extended this line of study to establish additional interactions with motion-defined contours. Only by examining interactions between different modalities can we hope to understand how the brain organizes complex scenes into unified percepts. The design of a model for luminancedefined illusory contour formation requires that one be aware of the larger visual scheme, allowing for future developments and extensions to integrate contours defined in various domains.

\section{CHARACTERISTICS OF ILLUSORY FIGURES}

At least three measurable perceptual characteristics are associated with illusory figures: contour strength, figure brightness, and figure depth. Strength is defined as the salience or clarity of the illusory boundary itself. Illusory brightness (or darkening) often accompanies illusory figures and is typically perceived as a homogeneous lightness increase within the illusory figure. Illusory figures are often perceived nearer to the observer than their inducers, thus the depth of the figure marks another consistently measurable characteristic.

Of the three measures of illusory contours, illusory brightening is the easiest to characterize and was the earliest to be quantified. In the first study of illusory brightening, Ehrenstein (1941) noted qualitatively a number of stimulus variations that affected brightness. A wide range of quantitative brightness reports followed, including measurements by rating scale and magnitude estimation (Dumais \& Bradley, 1976; Halpern, 1981; Jory \& Day, 1979; Petry et al., 1983), matching (Brussel, Stober, \& Bodinger, 1977; Spillman, Fuld, \& Neumeyer, 1984), nulling (Halpern, 1987; Spillman et al., 1984), and threshold increment levels (Coren \& Theodor, 1975; Dresp, 1993; Dresp \& Bonnet, 1991; Jory, 1987). Brightness measurements appear to be consistent across different types of measurement procedures (Dresp, 1992; Spillman et al., 1984). The most noticeable of illusory contour characteristics in such common figures as Kanizsa's and Ehren- 
stein's, illusory brightness received a great deal of early attention and was often invoked as the causal factor in illusory contour formation, as opposed to a consequence of illusory figure formation. Indeed, researchers have demonstrated a high degree of correlation between simultaneous brightness contrast and illusory brightening in edgeinduced illusory figures (Brigner \& Gallagher, 1974; Dresp, 1992; Dresp, Lorenceau, \& Bonnet, 1990), although brightness contrast alone is insufficient to explain the phenomenon (Coren \& Theodor, 1975; Frisby \& Clatworthy, 1975).

The depth of illusory figures was the next feature to receive widespread attention, as Coren (1972) claimed for it an important explanatory role in illusory contour formation. Subsequent studies using rating and magnitude scales (Bradley \& Dumais, 1984; Halpern, 1981; Salzman \& Halpern, 1982; Whitmore et al., 1976), matching (Coren \& Porac, 1983), and nulling (Gregory \& Harris, 1974; Whitmore et al., 1976) verified that traditional edge-induced illusory figures (such as Kanizsa's figure) appear to hover in front of the inducers for most observers. There is some evidence that line-end illusory figures also appear stratified in depth, although to a lesser extent than do their edgeinduced counterparts (Coren \& Porac, 1983). Great store was originally placed in the perceptual salience of the depth effect, because it was apparently strong enough to invoke size constancy for small elements inside the illusory figure (Coren, 1972; Parks, 1985; Porac, 1978), but in subsequent studies it was pointed out that the Ebbinghaus effect--in which the perceived size of a central form is mediated by the size of surrounding elements-was at least partially responsible for the original findings (Parks, 1987; Predebon, 1985). Nevertheless, depth effects are generally robust and are associated with most illusory figures.

Most researchers in the modeling community are concentrating on modeling the strength or clarity of illusory contours. Unfortunately, strength is by far the most difficult of the three measures to define. Whereas brightness and depth, terms applicable to generic visual scenes, have standard and well-specified definitions, it seems that the only consistent definition given to strength is "that characteristic which is not brightness or depth." Observers have been instructed to rate strength (Day \& Kasperczyk, 1983), salience (Dumais \& Bradley, 1976), clarity (Halpern, 1981; Shipley \& Kellman, 1992a), distinctness/sharpness (Petry et al., 1983), perceived contrast (Banton \& Levi, 1992), and several other variants of these terms, while matching procedures have used benchmark stimuli of questionable equivalence. Subjects in different experiments not only are provided with a variety of clarity definitions, but often are given only vague indications of what exactly they are rating. In spite of this lack of consistency and specificity, there are few examples of subjects reporting confusion about what to look for or rate. In addition, data appear to be relatively consistent across subjects and experiments done with different methods and definitions, although there has been no systematic study of the correlation between the various definitions and measurement techniques. Establishment of a standard definition of contour strength and an associated measurement technique would be invaluable for ensuring the reliability of future psychophysical experiments. Even with carefully defined measures, ratings of illusory contour strength are notorious for extravagant intersubject variations, particularly among naive subjects. Illusory contours represent a unique and unfamiliar form of visual stimulation, so it is not surprising that many inexperienced subjects have difficulty establishing coherent determinations of contour strength.

What exactly are subjects rating in strength/clarity experiments? Are all the definitions above synonymous? "Strength" and "salience" are measures of perceptual recognizability and pronouncedness, while "sharpness" is a measure of spatial spread. The other common terms, "clarity" and "distinctness," fall somewhere in between these two extremes. Surely pronouncedness and spatial spread are at least somewhat related, perhaps explaining the consistency between subjects given different definitions, but they do not represent the same illusory contour measure. Diffuse illusory contours, such as those of Figure 5, have very fuzzy (unsharp) boundaries (Kennedy, 1976). A weak illusory contour does not appear to have this type of fuzziness, as would be required by a "sharpness" definition, but rather has less perceptual wallop (or salience). Because of the apparent consistency across subjects given different definitions of contour strength, the difference between these two definitions is not of paramount importance for psychophysics, but it does have serious implications for the representation of illusory contours in neural models - amplitude (or energy) of neural activity and degree of spatial spread are distinctly different quantities. To date, all neural models have implicitly employed the former representation, not even considering the alternatives. For the purpose of this paper, I will use the terms "strength" and "clarity" interchangeably in discussing this illusory contour measure. It is reasonable to assume that contour sharpness represents a fourth characteristic of illusory figures. However, because of the apparent high degree of correlation between strength and sharpness, and the lack of empirical studies of sharpness per se, there will be no further explicit discussion of illusory contour sharpness in this paper.
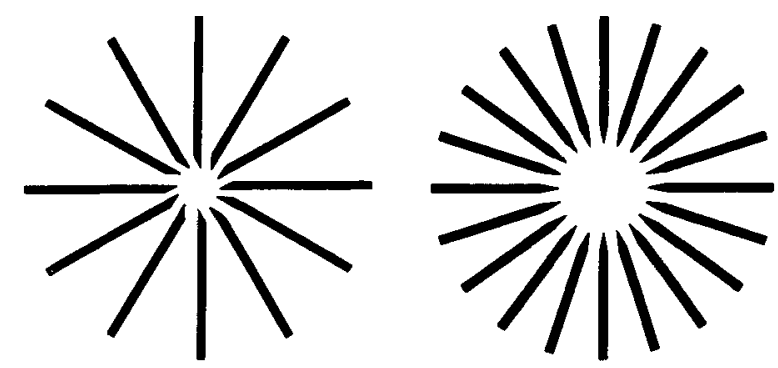

Figure 5. Diffuse illusory contours have brightness, but no clarity or depth. From "Subjective Contours, Contrast, and Assimilation," by J. M. Kennedy, 1979, in C. F. Nodine and D. F. Fisher (Eds.), Perception and Pictorial Representation (pp. 167-195, Figure 9.12), New York: Praeger. Copyright 1979 by Praeger Publishers. Reprinted with permission of Greenwood Publishing Group, Inc., Westport, CT. 
While brightness, depth, and clarity all mark measurable traits of illusory figures, there has been little agreement about their independence or their role in illusory contour formation. Coren (1972) claimed that perceived differences in depth drove illusory contour formation and thus determined brightness and clarity. Others claimed that brightness drove the formation and thus determined depth and clarity (Brigner \& Gallagher, 1974; Brussel et al., 1977; Frisby \& Clatworthy, 1975). Cognitivists and Gestaltists made the claim that boundaries were formed first, followed by depth and brightness (Gregory, 1972; Kanizsa, 1955; Rock \& Anson, 1979). We can now safely say that all these narrow views are incorrect. There are illusory contours without depth or brightness (Ware \& Kennedy, $1977,1978 \mathrm{a}$ ), illusory figures without clarity or depth (as in Figure 5) (Kennedy, 1976, 1978b, 1979, 1981; Meyer \& Dougherty, 1987; Parks, 1981, 1984; Richardson, 1979), illusory contours with clarity and depth but no brightness (Coren, 1972; Prazdny, 1983; Shapley \& Gordon, 1987), and illusory contours with clarity and brightness but no depth (Ware \& Kennedy, 1978a). In short, every combination but those involving depth without clarity has been observed. There is a great deal of independence between clarity, brightness, and depth. Watanabe and Oyama (1988) employed the causal inference method to determine to what degree, if any, these measures depended on one another. They found depth to be dependent on both clarity and brightness, and brightness to be somewhat dependent on clarity. However, of the three illusory figure characteristics, illusory brightness is most directly related to a reasonably well-understood, low-level phenomenafor edge inducers only, the degree of brightening is highly correlated with the degree of simultaneous brightness contrast near the inducers (Dresp, 1992; Dresp et al., 1990).

\section{DETERMINANTS OF ILLUSORY CONTOUR STRENGTH}

The definition of edge inducers included the constraint that the layout of illusory contour inducers must be locally consistent with occlusion of the inducers by the illusory figures (or with self-occlusion). This characteristic, obviously related to the ecological significance of illusory forms, is a general requisite for the formation of illusory contours, but there are also many specific determinants of an illusory contour's strength and an illusory figure's brightness. The structural determinants of contour strength can be divided into two classes. The first of these encompasses simple, configurational factors of the inducing elements, and thus I refer to these factors as low-level. Lowlevel stimuli determinants are describable in concrete, geometric terms without recourse to such perceptual abstractions as completeness or depth. In general, low-level factors can be varied parametrically. Luminance, spatial alignment, spatial extent, separation, and quantity of inducers are all examples of low-level determinants. Clarity and brightness typically vary smoothly with incremental variations in these parameters, making low-level determinants suitable for parametric investigation of their indi- vidual roles in contour formation. An overview of the current psychophysical data on low-level determinants of illusory contours is provided in the following section.

In contrast to low-level determinants, high-level determinants consist of configurational factors generally associated with cognitive phenomena. These factors cannot be defined in simple geometric terms, but require reference to abstractions and perceptual definitions. The degree of completeness of the inducing elements is an oft-cited example of a high-level determinant. Also in this class are the perceived depth of the figure and the effects of perceptual set, attention, memory, and recognizability of the illusory figure. Many of these complex factors cannot easily be varied parametrically, but still assist in revealing the internal representation of illusory contours. An overview of high-level determinants is provided after the summary of low-level factors, followed by a discussion of the ecological significance of both types of determinants. The existing neural models of illusory contour formation have been concerned primarily with modeling of specific lowlevel determinants. However, development of the next generation of models (or model extensions) will require an understanding of high-level determinants, as well as of how the models should be integrated into a comprehensive visual processing architecture.

\section{Low-Level Determinants}

The low-level determinants discussed below are, for the most part, well-quantified findings whose specifications engender a general acceptance in the illusory contour research community. In particular, in the domain of edgeinduced illusory contours, the effects of inducer spatial extent and position, inducer luminance, and alignment of inducers are understood to a degree commensurate for quantitative modeling. These parameters have not all been well quantified for line-end inducing stimuli, however, limiting the degree to which accurate specification of the operational characteristics of models of contour formation is possible. In addition, a more thorough understanding of such subtle effects as interscale interactions and the relation between edges and line ends is required so that the structure and mechanisms of models of illusory contour formation can be constrained further.

Spatial extent and proximity of inducers. The role of the spatial extent (or retinal size) of the inducing elements has always been a topic of intense research, but the recent publications of Shipley and Kellman (1992b) and Banton and Levi (1992) indicate a renewed interest in this area. In discussing inducer spatial extent, one must recognize that, for a given stimulus, several spatial factors may be varied. For example, in a Kanizsa figure, the inducers may be moved apart with no change in size, the inducers may be enlarged without movement, or the entire stimulus may be enlarged. These variations are depicted in Figure 6. Note that these three types of spatial variation are not independent - any one type can be achieved by manipulations of the other two varieties. For example, a decrease in the retinal size of the figure can be achieved through decreases of appropriate proportions in both inducer spacing and ra- 


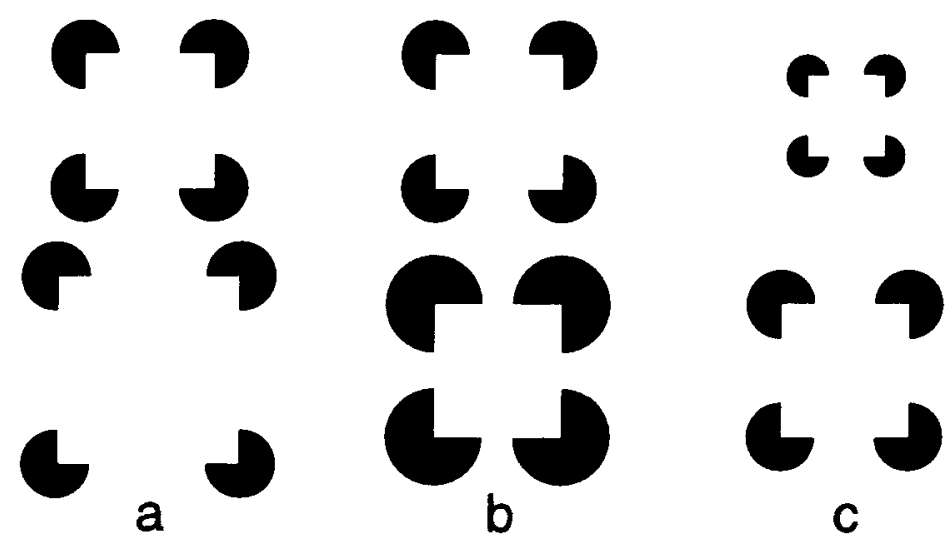

Figure 6. Spatial variations of edge inducers: (a) inducer spacing, (b) inducer radius, (c) retinal size.

dius. Early studies tended to be concentrated on a single set of spatial variations, but more recently, researchers have attempted to integrate all three sets into a single determinant.

Dumais and Bradley (1976) first studied the effect of retinal size on contour strength, finding that strength decreased monotonically for Kanizsa triangles with increasing retinal size (from $1.2^{\circ}$ to $18.9^{\circ}$ of visual angle). Employing Ehrenstein figures, Siegel and Petry (1991) produced similar results for large visual angles-a decrease in both clarity and brightness-but found that clarity actually increased as retinal size increased from $0.25^{\circ}$ to $1.5^{\circ}$ when the Ehrenstein figure had eight or more inducing lines. Watanabe and Oyama (1988) found that clarity and brightness decreased monotonically as they increased the separation between the sectored circle inducers in a Kanizsa figure without varying any other spatial parameters. By varying the width of inducing lines in an Ehrenstein figure, Petry et al. (1983) and Sambin (1985) found that clarity was a monotonically increasing function of the width of the inducers, while brightness exhibited an inverted-U shape. Dresp (1992) investigated the effect of Kanizsa (edge) inducer separation and size on illusory brightness, finding that brightness was an inverse function of inducer proximity and a positive function of inducer size. In general, these studies have indicated that both illusory contour strength and illusory figure brightness increase with increasing degrees of inducer support and decreasing distance between inducers.

Shipley and Kellman (1992b) have attempted to unify inducer separation, inducer size, and retinal size of the stimuli into a single determinant. They posit that the only relevant spatial factor is the ratio of the length of supported contour to the length of the entire illusory contour, a parameter referred to by Lesher and Mingolla (1993) as the support ratio. Shipley and Kellman (1992b) created stimuli similar to those of Figure 6 by varying retinal size, inducer spacing, and inducer size independently, and had subjects rate the resulting illusory contour clarities. Like Watanabe and Oyama (1988), they found that clarity decreased with increasing inducer spacing and decreasing inducer size. However, in constrast to Dumais and Bradley
(1976) they observed no effect of retinal size. Shipley and Kellman (1992b) verified this controversial finding by performing forced choice experiments in which pairs of stimuli were presented that differed only in retinal size. Note that the stimuli employed resulted in illusory figures that ranged in retinal size only from $0.7^{\circ}$ to $2.8^{\circ}$, so that there was little overlap with the larger retinal sizes employed by Dumais and Bradley. By plotting clarity ratings as a function of the support ratio, which in the case of a Kanizsa square is twice the inducer radius divided by the center-to-center inducer separation, Shipley and Kellman (1992b) discovered that clarity was a linearly increasing function of the support ratio, at least in the case of solid, edge inducers.

Banton and Levi (1992) performed similar experiments by varying Kanizsa figure inducer separation and inducer size. They present experimental results which indicate that local support ratio - the support ratio along each side of a Kanizsa rectangle- is the determining factor of contour strength, as opposed to a global support ratio which would encompass the perimeter of the entire illusory figure. In these experiments, Banton and Levi established that contour clarity, as measured by a matching procedure, was an increasing function of this local support ratio. This relation was monotonic but not linear, in contrast to Shipley and Kellman's (1992b) findings. Clarity was roughly a linear function of support ratio when plotted on a log-log scale, implying that clarity and support ratio are related by a power function:

$$
\text { clarity } \propto[1-\text { support ratio }]^{-1.48} \text {. }
$$

This curve matches the linear function of Shipley and Kellman reasonably well for low and intermediate support ratios, but it diverges significantly for high support ratios. A possible source of this variance is the difference in clarity measurement techniques-Banton and Levi used an unconventional technique wherein the luminance of a real line was matched to the illusory contour; Shipley and Kellman used rating scales. Also, Banton and Levi's parametric fit seems unduly biased by several entries at very low support ratios. Although the data of the two research groups 
do not coincide perfectly, both indicate the same general trend for edge inducers: a monotonic increase in contour clarity as support ratio increases.

The effect of support ratio on line-end induced contour strength has been insufficiently studied, but the data of Petry et al. (1983) indicate that it may also play an important role in determining contour strength in these cases as well. Employing stimuli similar to those of Figure 7, and excepting very thin lines, they found clarity to increase to a saturated plateau with increasing support ratio, defined for their Ehrenstein figures as the product of the number of lines and line width, divided by the illusory figure circumference. This effect has been replicated by Sambin (1985), using an Ehrenstein figure, but it has been contradicted by recent studies done with other forms of illusory stimuli (Lesher \& Mingolla, 1993; Soriano, Spillman, \& Bach, 1994). Employing a phase-shifted line grating as in Figure 2c, Soriano et al. (1994) found that contour saliency tended to decrease monotonically with increasing line width. Primary differences between Ehrenstein figures and grating figures include the curvedness of the contour in the former and the lack of illusory brightness in the latter, but it is not clear whether either of these differences accounts for the discrepancy in the data.

Lesher and Mingolla (1993) employed variants of figures first presented by Varin (1971), consisting of a Kanizsa square with concentric circles substituted for the solid circle inducers, as depicted in Figure 8. By independently varying the width $(8 a \rightarrow 8 b)$ and number of lines $(8 b \rightarrow 8 c)$, one can study the effects of inducer width, support ratio, and number of inducers. With many thin inducing lines, the Varin figure consists solely of line-end inducers, whereas with a single thick inducing line for each sectored circle, the figure consists solely of edge inducers. Lesher and Mingolla found that contour strength and illu- sory brightness increased monotonically with increasing line width, for a constant number of lines. However, neither strength nor brightness varied monotonically with support ratio- support ratio was not a reliable predictor of either quantity, exhibiting varied trends for different numbers of lines and inducer radii. Therefore, Shipley and Kellman's (1992b) hypothesis that support ratio determines contour strength is invalid for line-end inducers. The duality of edges and lines (edges as thick lines, lines as thin edges) leaves some doubt about the validity of support ratio theories in all but the most constrained circumstances.

Number of inducers. The number of inducing elements has close relation to the spatial extent of the inducers-both factors affect the support ratio, especially for edge inducers. Using solid semicircular inducers of varying number and size, Mast and Fox (1994) demonstrated that contour clarity increases monotonically with increasing support ratio, independently of the number of edge inducers. Although this has been the only explicit study of the effect of the number of edge inducers, there have been several investigations of the number of line-end inducers on clarity and brightness, using both thin and thick lines. As is indicated in Figure 7, Petry et al. (1983) varied the number, as well as the widths, of inducing lines in Ehrenstein figures. They found that increasing the number of lines had very little effect on contour clarity but resulted in a monotonic increase in brightness. This finding conflicts with those of a number of other researchers, all of whom report a monotonic increase in both clarity and brightness with increases in the number of lines in Ehrenstein figures (Ehrenstein, 1941; Frisby \& Clatworthy, 1975; Sambin, 1985; Siegel \& Petry, 1991). The difference in results probably stems from the particular line widths used. Petry et al. employed a series of different line widths, most of which were thicker than those used by the other exper-

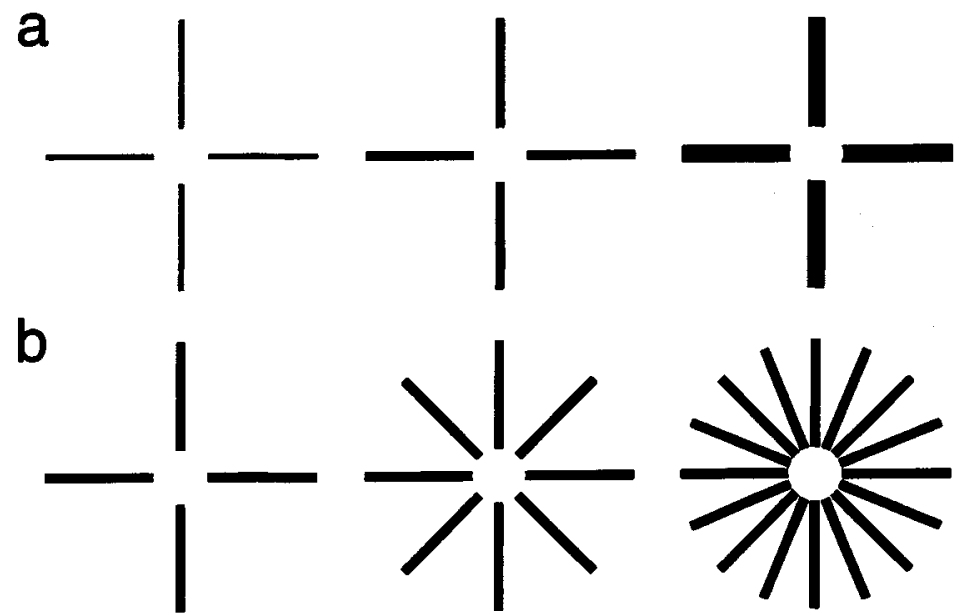

Figure 7. Increasing support ratio by (a) increasing the line widths for a constant number of lines and (b) increasing the number of lines for a constant line width. From "Stimulus Determinants of Brightness and Distinctness of Subjective Contours," by S. Petry, A. Harbeck, J. Conway, and J. Levey, 1983, Perception \& Psychophysics, 34, pp. 169-174 (Figure 1). Copyright 1983 by Psychonomic Society, Inc. Reprinted with permission. 
a
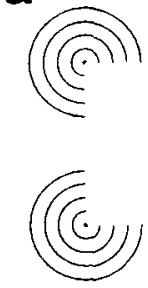

b
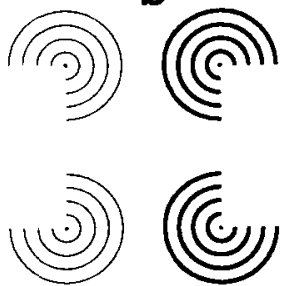
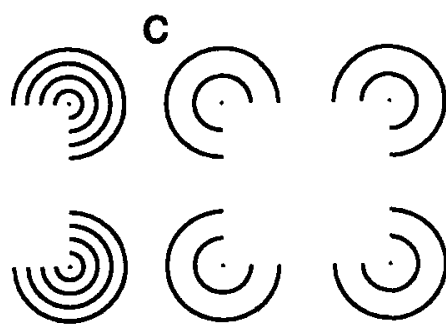

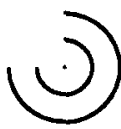

Figure 8. Variants of figures presented by Varin (1971) provide a means by which to examine the roles that line ends and edges play in illusory contour formation.

imenters. In the restricted case of very thin lines, Petry et al. did observe that the number of lines affected clarity, although not as pronouncedly as was observed in the other experiments. Soriano et al. (1994) confirm a monotonic increase in contour saliency with increases in the number of lines in phase-shifted grating figures.

With the stimuli of Figure 8, however, Lesher and Mingolla (1993) found that for constant line widths, both contour strength and illusory brightness were inverted-U functions of the number of inducing lines. Soriano et al. (1994) have observed a similar trend while holding the number of lines constant and varying the spacing between adjacent lines. In Lesher and Mingolla's experiment, line spacing necessarily decreased when the number of lines increased, owing to the constraint that the largest of the concentric circles always retained the same radius. In the experiment of Soriano et al., the extent of the contour necessarily increased with increasing line spacing, owing to the constraint that the number of lines remained constant. In both experiments, possible confounds with the other interdependent factors render it impossible to determine which factor or factors are responsible for the inverted $U$. Additional experiments with better controls and interdependence analyses are required in order to tease out the effects of line spacing, number of lines, and extent of the contour on illusory contour strength and illusory brightening. In any event, the inverted $U$ ranks as an important and surprising new finding that must be reconciled with neural models of contour formation.

Inducer luminance and contrast. The effect of inducer luminance and contrast on illusory contour clarity and brightness has been a topic of lively debate. Dumais and Bradley (1976) initially found that contour clarity increased with decreasing ambient illumination of a Kanizsa figure, a finding supported by Brussel et al. (1977). However, these results have been cast into doubt by Warm, Dember, Padich, Beckner, and Jones (1987), Spillman et al. (1984), and others (Parks \& Marks, 1983; Watanabe \& Oyama, 1988). Warm et al. found that the clarity of a Kanizsa figure decreased with decreasing illumination, while Spillman et al. (1984) discovered the same relation between illusory brightness and illumination in an Ehrenstein configuration. Warm et al. hypothesize that subjects in the experiment of Dumais and Bradley (1976) were rating illusory contour clarity with respect to real contour clarity/sharpness at the same illumination level. Although the absolute clarity decreased with illumination levels, Warm et al. also observed that the clarity of illusory contours increased relative to that of real contours.

Several studies have shown that there need be no global agreement between inducer contrasts to instantiate illusory contours (Brigner, 1982; Grossberg \& Mingolla, 1985a; Kellman \& Loukides, 1987; Prazdny, 1983; Shapley \& Gordon, 1987). That is, not all the inducers need be of the same direction of contrast; alternating dark and light inducers on an intermediate (gray) background are also efficacious, as is indicated by the stimuli of Figure 9. Prazdny (1983) originally reported that in the stimuli of Figure $9 \mathrm{a}$, illusory brightness was markedly diminished, if not completely eliminated. Hershberger and Stallard (1984) reported that, paradoxically, Prazdny's figure actually appeared brighter than it would if composed of all dark inducers. They defined a parameter, called "contrast variability," which takes into account not only the contrast between the inducers and the background, but also that between the individual inducers. Hershberger and Stallard reported that illusory brightness increased with increasing contrast variability, but they failed to control for the effect of spatial inducer variations: To change the contrast variability they moved the boundaries between the dark and light portions of the inducers, thereby altering the spatial configuration as well as the contrast. More recently, Kellman and Loukides (1987) have employed stimuli similar to that of Figure $9 \mathrm{~b}$ to demonstrate that illusory brightness may be completely eliminated by allowing subjects to balance the luminance of the positive and negative contrast inducers. The exact nature and extent of brightness effects in such mixed-contrast stimuli remain an open issue.

Although there need be no contrast agreement between inducers, local contrast between inducer and background is extremely important. Most researchers have reported the absence of illusory contours when inducers are isoluminant with the background (de Weert, 1983; Gregory, 1977; Livingstone \& Hubel, 1988; Watanabe \& Sato, 1989), although Ejima and Takahashi (1988) noted that illusory contours generated by abutting line gratings (as in Figure 2c) can be perceived under certain isoluminant conditions. De Weert (1983) presented a detailed study of the effect of color and color differences on illusory contour formation.

Inducer alignment. Kanizsa (1955) noted that one of the conditions for the formation of illusory contours was proper alignment between the supporting edges of the in- 


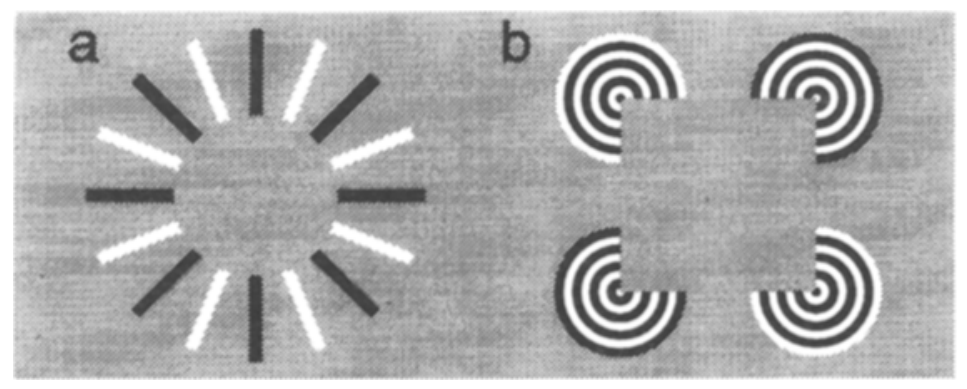

Figure 9. Inducing elements of varying contrast polarity can generate strong illusory contours with limited amounts of illusory brightening. (a) Prazdny's (1983) influential figure. (b) Stimulus similar to those employed by Hershberger and Stallard (1984) to demonstrate that there can be illusory brightening associated with such figures. Figure 9a is from "Illusory Contours Are Not Caused by Simultaneous Brightness Contrast," by K. Prazdny, 1983, Perception \& Psychophysics, 34, pp. 403-404 (Figure 1B). Copyright 1983 by Psychonomic Society, Inc. Figure $9 \mathrm{~b}$ is adapted from "Contrast Variability Lightens Subjective Figures," by W. Hershberger and S. Stallard, 1984, Perception \& Psychophysics, 36, pp. 92-94. Copyright 1984 by Psychonomic Society, Inc. Figure $9 \mathrm{a}$ is reprinted, Figure $9 \mathrm{~b}$ adapted, with permission.

ducers - that is, that illusory contours are observed only when the continuation of each supporting edge meets another supporting edge. This continuation reflects the curvature of the supporting edges, as is indicated by the curved illusory figure of Figure lc. Kanizsa presented only stimuli in which the continuations from the different supporting edges coincided perfectly. For example, perfectly aligned straight-edged inducers led to straight illusory contours, and perfectly aligned curved-edged inducers led to curved illusory contours. By presenting Figure $10 \mathrm{a}$, in which straight inducers lead to curved illusory contours rather than straight contours meeting at a vertex, Pastore (1971) and Gregory (1972) demonstrated that illusory contour formation involved more than simple continuation of supporting edges, a finding confirmed by others (Bross \& Michelangeli, 1988; Rock \& Anson, 1979; Ship- ley \& Kellman, 1992a). Misalignment of straight inducing edges, however, weakens the resulting illusory contour.

Bross and Michelangeli (1988) attempted to quantify the effect of edge misalignment by studying the time that it took subjects to perceive an illusory form when presented with Kanizsa stimuli having inducers rotated so that the edges of the missing sectors did not align. Reaction delays lengthened as alignment decreased, allegedly indicating weakened contours. In a more comprehensive and convincing paper, Shipley and Kellman (1992a) presented experiments in which the inducers of a Kanizsa square were shifted laterally, with concomitant variation of orientation of the missing inducer sectors, as depicted in Figure $10 \mathrm{~b}$. They found that contour strength decreased with misalignments and that it was highly correlated with what they refer to as inducer "relatability." Supporting a
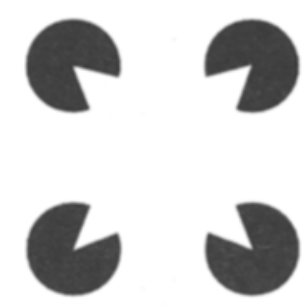
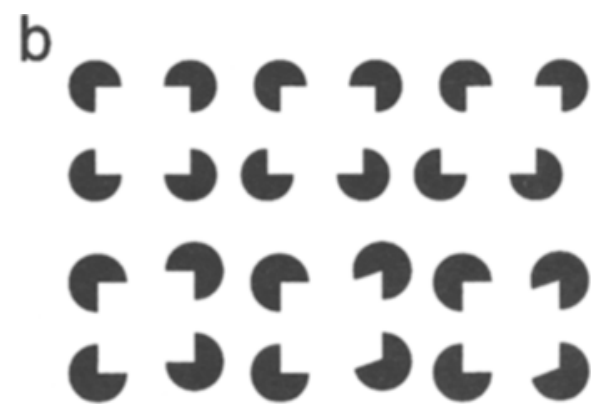

Figure 10. Curved illusory contours can result when two straight supporting edges are misaligned. (a) Gregory's (1972) original stimulus. From "Cognitive Contours," by R. L. Gregory, 1972, Nature, 258, pp. 51-52 (Figure 3). Copyright 1972 by Macmillan Magazines Limited. Reprinted with permission. (b) Shipley and Kellman (1992a) varied the degree and type of edge misalignment to determine how well subjects' ratings of contour strength corresponded with "relatability" of the edges. From "Perception of Partly Occluded Objects and Illusory Figures: Evidence for an Identity Hypothesis," by T. F. Shipley and P. J. Kellman, 1992, Journal of Experimental Psychology: Human Perception \& Performance, 18, pp. 106-120 (Figure 6). Copyright 1992 by American Psychological Association. Reprinted with permission. 
edges are relatable if their extensions intersect with an oblique angle, as Figure 11 depicts (Kellman \& Shipley, 1991). Contour strengths are determined by the degree to which the relatability criteria are fulfilled, as well as by the acuteness of the intersection angle-the more acute the angle, the weaker the contour. Note that Kellman and Shipley do not claim that the illusory contours take the simple piecewise-linear paths used to determine relatability. The role of relatability in Kellman and Shipley's model of illusory contour formation is discussed further in the present paper in the section on Neural Models of Illusory Contour Formation.

For line-end inducers, the resulting illusory contour is roughly perpendicular to the inducing lines. Inducer alignment for this type of stimulus refers to alignment between perpendiculars to the line ends. Kennedy (1978a) varied the slant of the inducing lines in an Ehrenstein figure from perpendicular to the edge of the illusory circle to tangent to the circle, noting that illusory brightness and clarity decrease monotonically with this alteration. However, there are many examples of efficacious line-end inducers that are not perpendicular to the illusory contour (Frisby \& Clatworthy, 1975; Gillam, 1987; Gregory, 1987; Halpern, Salzman, Harrison, \& Widaman, 1983; Parks, 1980; Rowbury, 1982). In fact, recent evidence shows that under certain conditions, perpendicular elements are inferior to oblique and acute intersections (Gillam \& Goodenough, 1994). Not limiting line-end contour completion strictly to the perpendicular makes ecological sense; an occluded line conveys a decreasing amount of information about the orientation of the occluder as the line thickness approaches zero. However, if one makes the reasonable assumption that foreground and background position and orientation are independent, the probability that an edge or line in the background intersects a foreground edge at an angle between $\theta$ and $\theta+d \theta$ is proportional to $\sin \theta d \theta$ (Heitger \& von der Heydt, 1993). This implies that a perpendicular intersection is ecologically most likely, with probabilities decreasing as the angle of intersection becomes more acute or oblique.
The relative positions of line ends appear to play a greater role in determining whether illusory contours will appear than does the orientation of the lines with respect to the illusory contours. If an excessive amount of curvature is required in order to connect the line ends, there will most likely be no illusory contour (Gillam, 1987; Rowbury, 1982; Shipley \& Kellman, 1992a). In both edge and line-end induced contours, completion in the form of a smooth curve is apparently preferred to completion with curvature discontinuities (Gregory, 1972; Shipley \& Kellman, 1992a). However, in rare cases, illusory contours with unsupported corners or discontinuities may be observed (Coren, Porac, \& Theodor, 1986). For example, some observers report a square illusory figure in the Ehrenstein stimulus of Figure $2 b$, rather than a circle.

Small-scale facilitation and interference. Information on a scale much smaller than that of the inducing elements can have a profound effect on the resulting illusory contour. Propitious placement of small dots or short line segments, called anchors, can drastically affect the shape of an illusory figure and may increase contour salience, as is indicated in Figure 12 (Brady \& Grimson, 1981; Day \& Jory, 1980; Gerbino \& Kanizsa, 1987; Minguzzi, 1987; Rowbury, 1982), even though by themselves these anchors are incapable of generating illusory contours (Day \& Jory, 1980; Kanizsa, 1955; Zucker \& Davis, 1988). Gerbino and Kanizsa have noted that unlike normal inducing elements, which abut the illusory contour, anchors appear to lie within or along the contour. This observation, if taken in the context of the illusory figure occluding the inducers, may imply that anchors are perceived as belonging to the illusory figure rather than to the background. As yet, there have been no studies to determine whether this is the case, although Gregory and Harris (1974) demonstrated that the stereoscopic depth of anchor-like elements affects illusory contour strength in a manner consistent with this hypothesis. Anchors can relieve the constraint that illusory contours have only a limited degree of curvature between inducing elements by providing information about

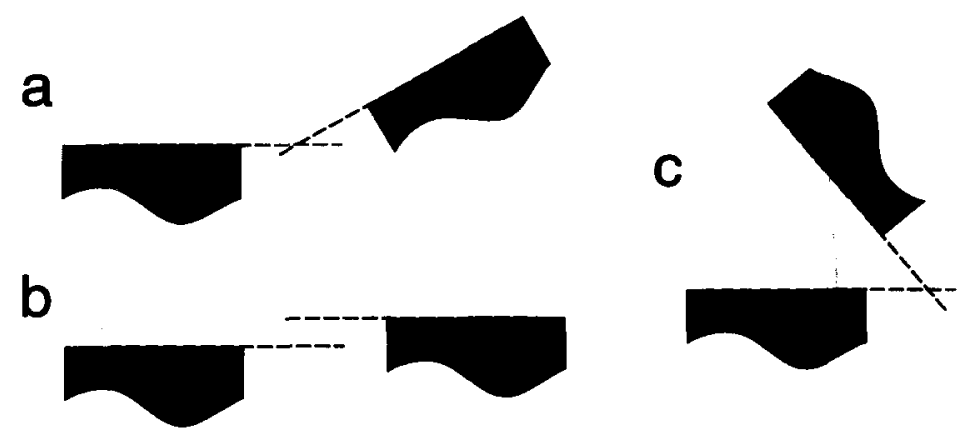

Figure 11. Two inducers are relatable if linear extensions of their supporting edges (or perpendicular extension of line ends) intersect with an oblique angle. The inducers in (a) meet this criterion and are thus relatable, but in (b) the extensions do not intersect and in (c) the intersection angle is acute, implying that the inducers are unrelatable in both these cases. From "A Theory of Visual Interpolation in Object Perception," by P. J. Kellman and T. F. Shipley, 1991, Cognitive Psychology, 23, pp. 141-221 (Figure 32). Copyright 1991 by Academic Press. Adapted with permission. 


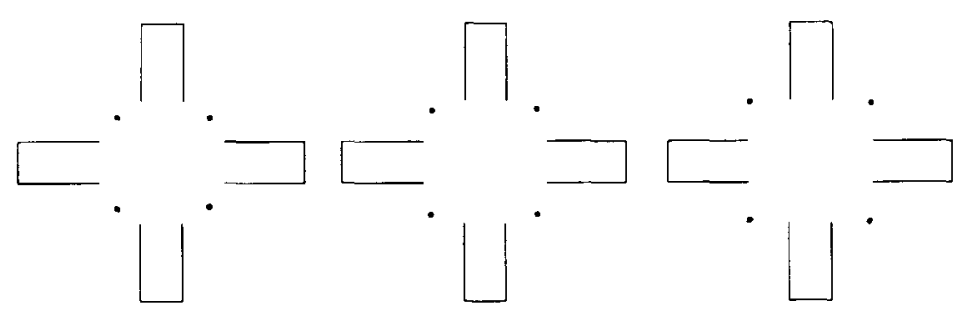

Figure 12. Day and Jory (1980) demonstrated that small point or dot "anchors" facilitate contour formation and change the shape of the illusory figure. From "A Note on a Second Stage in the Formation of Illusory Contours," by R. H. Day and M. K. Jory, 1980, Perception \& Psychophysics, 27, pp. 89-91 (Figure 2). Copyright 1980 by Psychonomic Society, Inc. Reprinted with permission.

where corners (curve discontinuities) should be located, as in the rightmost stimuli of Figure 12.

Conversely, small-scale information can also weaken illusory contours, or even prevent their formation altogether. With a series of striking images, similar to that of Figure 13a, Kennedy (1988) has demonstrated how altering the shape of a line end so that it appears beveled, pointed, or rounded can eliminate illusory contours (or at least render them diffuse, as in Figure 5). Interference from a small scale-the bevel of the line ends - destroys the illusory contour supported by larger scales - the system of collinear line ends. There are many other examples of the effect of line-end shape (Ehrenstein, 1941; Kennedy, 1978a, 1978b), but interference can also arise from unconnected scenic elements. Sambin (1987) and Pinna and Sambin (1991) have presented examples in which interference in Ehrenstein figures originates from small elements (dots and short lines) much like the facilitating anchors of Figure 12. In these cases, one example of which is depicted in Figure $13 \mathrm{~b}$, small dots or lines positioned near the inducers, close to the illusory contour, can significantly reduce clarity and brightness. Clarity decreases as the elements approach the illusory contour, whether the elements approach from the inside or from the outside. Sambin $(1981,1987)$ has produced similar results for disturbances within the interior of the inducing elements themselves. The delete- rious effect of multiple lines converging on the illusory border at the same location (such as to form a vertex) is also well established, even with very short line segments (Albert \& Hoffman, 1992; Kennedy, 1978b; Purghé, 1993).

Spatial discontinuities. Kellman and colleagues (Kellman \& Loukides, 1987; Kellman \& Shipley, 1991; Shipley \& Kellman, 1990, 1994) have made the strong claim that discontinuities in the first derivative of the inducers are essential to establishing illusory contours. These discontinuities take the form of abrupt changes in the orientation of the outer boundaries of the inducers - corners and line ends. By presenting pairs of similar stimuli with and without discontinuities, Shipley and Kellman (1990) demonstrated a marked decrease in illusory contour strength when discontinuities were absent. As Kellman and Loukides (1987) have argued, discontinuities are strong ecological clues to occlusion of the inducers. However, several researchers have demonstrated illusory contour formation in stimuli without apparent discontinuities by employing edge inducers with gradually tapering luminance gradients-blurry-edged inducers (Grossberg \& Mingolla, 1985a; Lesher \& Mingolla, 1993; Shapley \& Gordon, 1987). One could argue that these stimuli have discontinuities when boundaries are measured at a sufficiently large spatial scale, but this argument inevitably leads to discontinuities in every blurry-edged inducer, no matter how grad- a

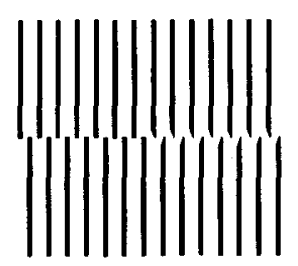

b

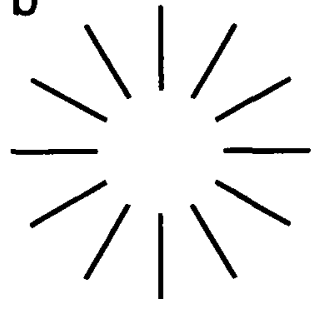

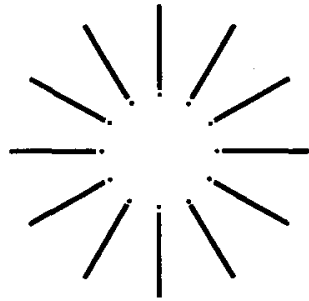

Figure 13. Small scale interference: (a) Beveled line ends destroy illusory contours. From "Line Endings and Subjective Contours," by J. M. Kennedy, 1988, Spatial Vision, 3, pp. 151-158 (Figure 1). Copyright 1988 by VSP International Science Publishers. Adapted with permission. (b) Small interfering dots decrease the clarity of the illusory contour. From "A Dynamic Model for Anomalous Figures: The Shape of Line-Induced Brightness Modifications," by B. Pinna and M. Sambin, Perception, 20, pp. 219-232 (Figure 7). Copyright 1991 by Pion Limited, London. Adapted with permission. 
ual the luminance gradient of the edges. A similar argument might be made for sharp-edged inducers: at sufficiently large scales regions of high curvature are indistinguishable from real discontinuities, so every sharp-edged stimuli would have discontinuities. Regardless of whether we accept the troublesome scale argument for blurry-edged inducers, or limit the domain to sharp-edged inducers, spatial discontinuities evidently represent a salient low-level determinant of illusory contour formation.

Inducer structure and figural interference. Kanizsa (1976) showed that if the solid inducers of his eponymous figure were replaced by outlined circles (with appropriate missing sectors), illusory contour formation was blocked. Figure 14 depicts this configuration, along with two other configurations in which illusory contours and illusory brightening are either eliminated or weakened considerably (Frisby \& Clatworthy, 1975; Kanizsa, 1974). There are no examples of illusory contours induction collinear to thin lines, as would be required for contour formation in the first two examples of Figure 14, although there have been examples of collinear lines in noninductive roles, such as the short line anchors employed by Minguzzi (1987). If one considers collinear lines as extremely thin edges, it appears that the thickness of the inducer (in the direction perpendicular to the support region) plays an important role in illusory contour formation, as has been supported by several parametric investigations (Brigner \& Gallagher, 1974; Purghé, 1991; Sambin, 1981). Purghé (1991) found contour strength to be an increasing function of this inducer thickness. For line-end stimuli, the lengths of the inducing lines are strong determinants of contour strength - longer inducing lines tend to result in stronger illusory contours (Ehrenstein, 1941; Redies \& Spillman, 1981; Sambin, 1985; Soriano et al., 1994).

Kanizsa (1974) noted that illusory contour strength was lessened by the addition of lines that penetrated the contour, as is depicted in Figure 14c. Similar findings have been reported in the context of line-end contours in which one or more of the inducing lines penetrates the illusory figure (Gregory, 1987; Soriano et al., 1994). Soriano et al. (1994) have shown that relatively small overlaps $\left(<0.1^{\circ}\right)$ of shifted gratings significantly decrease the probability of illusory contour detection. Note that if the interfering lines of Figure $14 \mathrm{c}$ are perceived as a "wire" square which is weaved around the illusory square, the illusory contours may remain salient-an indication that the perceptual depth ordering of various parts of the stimuli plays an important role in contour formation, as will be discussed further in the next section. Similarly, many researchers have noted that background texture can interfere with illusory contour formation if it is perceived as being behind the inducing elements (Ramachandran, Ruskin, Cobb, Rogers-Ramachandran, \& Tyler, 1994; Reynolds, 1981; Rock \& Anson, 1979).

\section{High-Level Determinants}

High-level determinants are typically more difficult to quantify than their low-level counterparts. The effects of stereoscopic depth modification of the illusory figures, suitable for parametric investigation and thus perhaps more suitably a low-level determinant, are included within the more abstract categorization of "depth modifications"-aspects of which are decidedly high level and poorly understood. None of the high-level determinants has yet been addressed in a fully functional model of illusory contour formation, although certain aspects of the effect of depth and stratification of inducers and illusory figures on the existence of illusory contours have been discussed (Grossberg, 1987a, 1994; Kellman \& Shipley, 1991; Sajda \& Finkel, 1995). Although the apparently "cognitive" nature of some of the high-level determinants preclude them from detailed simulation-at least until the cognitive processes themselves are understood bettermany of the effects discussed below are amenable to modeling. Even in cases where the underlying cognitive processes are currently unmodelable, one is not prevented from modeling the effects of the results of these processes on illusory contour formation. a

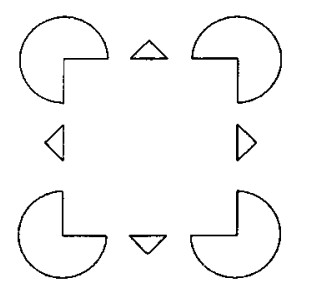

b
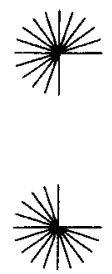
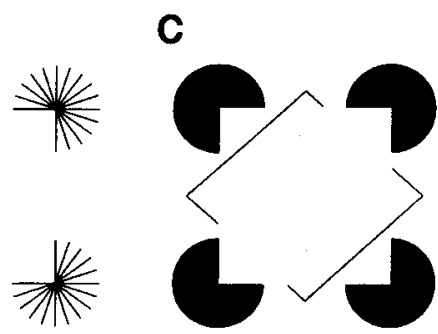

Figure 14. Figural interference effects: (a) Outlining a Kanizsa figure eliminates illusory contours. (b) Contour induction collinear to thin inducing lines does not occur. (c) Lines intersecting illusory contours can decrease contour strength. Figures 14a and $14 \mathrm{c}$ are from "Contours Without Gradients or $\mathrm{Cog}$ nitive Contours?" by G. Kanisza, 1974, Italian Journal of Psychology, 1, pp. 107-123 (Figures 29 and 35). Copyright 1974 by Societa Editrice il Mulino. Adapted by permission. Figure 14b is from "Illusory Contours: Curious Cases of Simultaneous Brightness Contrast," by J. P. Frisby and J. L. Clatworthy, 1975, Perception, 4, pp. 349-357 (Figure 8b). Copyright 1975 by Pion Limited, London. Adapted by permission. 


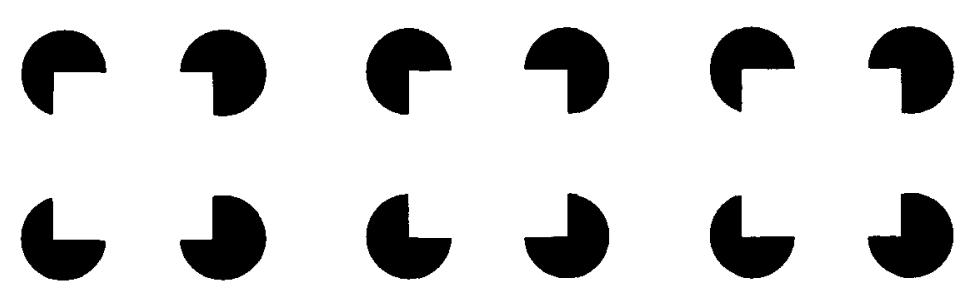

Figure 15. Stereoscopic manipulation of illusory figure depth affects the illusory contour strength.

Depth modifications. Observers usually perceive illusory figures as being raised from the background, floating above (and occluding) the inducing elements (Coren, 1972; Kanizsa, 1955). Thus, one might wonder what effect the perceived depth of the figure has on the strength of associated illusory contours. Figure 15 depicts a Kanizsa figure which, when stereoscopically fused, has disparity information indicating that it lies in front or behind the inducers, depending on which pair of stimuli are fused. In the former case, the illusory contour looks much stronger than the monocular contour, whereas in the latter, it looks much weaker. These types of stimuli were employed by Simmonds (1974), who had subjects rate contour strength as disparity was varied. Simmonds found no effect on strength when the figure was made to appear in front of the inducers, but did find that strength was decreased when the figure was made to appear in back-when the cues were not consistent with an occluding illusory figure. However, subsequent research has shown a reliable effect of both crossed and uncrossed disparities (Lawson et al., 1974; Nakayama, Shimojo, \& Ramachandran, 1990; Whitmore et al., 1976). When one uses a binocular stimulus with no disparity as a baseline, contour strength increases monotonically to a saturated maximum as figure depth decreases (nearer to the observer), with the inverse holding true as figure depth increases. Gregory and Harris (1974) report similar results with line-end inducer configurations.

In changing the disparity information of the illusory figure, the inducers themselves are slightly altered, leading to the possibility (albeit a small one) of confound between the effects of disparity and those of inducer structure. However, indirect depth modifications that leave the inducer structure unchanged have been employed to unequivocally demonstrate the effect of perceived depth on contour salience. Rock and Anson (1979) constructed a display, similar to that of Figure 16, in which a coarse texture was printed over the entire background of a Kanizsa figure. Under monocular conditions, no illusory contour was perceived, apparently because of figural interference from the texture. The same was true if the texture was stereoscopically located in back of the inducers (uncrossed fusion of the middle and right images of Figure 16). If the texture was stereoscopically placed in front of the inducers (uncrossed fusion of the left and middle images), however, the illusory contour became clear. This last case is consistent with a semitransparent texture lying in front of both the inducers and the illusory figure, allowing stratification of illusory figure and inducers to occur without paradox. When this texture is behind the stimuli, however, the observer has the perception of looking through the central region of the stimuli, and thus the system is not consistent with an occluding figure (since the illusory figure would need be transparent to the texture but opaque to the inducer). This example, and others in the same vein (Nakayama et al., 1990; Reynolds, 1981), show that the perceived consistency with global occlusion can have a profound effect on contour strength. Using stimuli similar to those of Rock and Anson (1979) - periodic textures overlying conventional illusory contour inducing stimuli- $-\mathrm{Ra}-$ machandran and Cavanagh (1985; Ramachandran, 1986a, 1986b, 1987) have demonstrated "capture" of the texture by the illusory figure when the texture has ambiguous stereoscopic depth. That is, within the illusory figure the texture appears to be at the same depth as the figure is, while

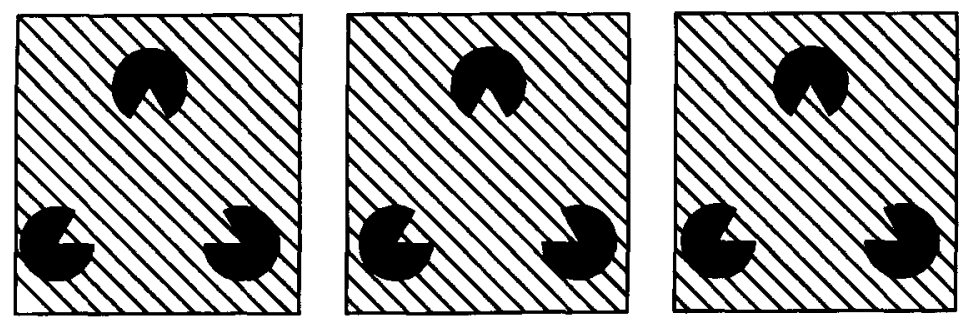

Figure 16. Rock and Anson (1979) showed, using the physical equivalent of this stereogram, that the perceived depth of the diagonal texture determines whether or not an illusory contour will be formed. The texture appears in front of the inducers if the left and middle images are fused (uncrossed), and illusory contours appear. When the middle and right images are fused, the texture appears in back of the inducers, and illusory contour formation is blocked. 
elsewhere it appears to be at the same depth as is the background. Ramachandran (1986a) and Ramachandran et al. (1994) point out that even in certain monocular stimuli, regular background textures can be captured by the illusory figure to appear at the same depth as that of the figure.

Despite the findings of Rock and Anson (1979), consistency with occlusion of the inducers by the illusory figure is not a prerequisite for perception of a salient contour. Strong illusory contours have been demonstrated in the absence of perceived depth by employing self-occluding stimuli (Kellman \& Shipley, 1991; Kennedy, 1979; Kennedy \& Lee, 1976; Parks, 1986) and three-dimensional stimulus constructs (Ware \& Kennedy, 1977, 1978a). The phase-shifted grating of Figure 2c, one of the most frequently employed illusory stimuli, is itself not entirely consistent with global occlusion - one side of the grating must be perceived in front of the other, so that side's line ends are not occluded at all. Purghé (1993) has constructed a clever series of pictorially 3-D stimuli decidedly inconsistent with illusory figure occlusion, which still give rise to strong illusory contours and illusory brightening. Purghé's examples were designed to refute the claims of Parks and Rock (1990), who argued that 3-D stimuli inconsistent with occlusion did not give rise to illusory contours.

The examples in the literature which seem to indicate that contours are destroyed by inconsistency with an occluding illusory figure can be discounted on the grounds of other monocular effects-small-scale or figural interference, as observed in the stimuli of Parks and Rock (1990) and Rock and Anson (1979), respectively. It does appear, however, that providing monocular cues that indicate that the stimuli are indeed consistent with occlusion can facilitate instantiation of illusory contours which would otherwise be blocked by these interference effects. Although this asymmetry between contour instantiation and destruction may be resolved by demonstration of contour destruction solely on the grounds of (monocular) occlusion inconsistency, there have been no such convincing displays or experiments yet. As mentioned above, however, there is no such asymmetry in the case of stereoscopically altered stimuli: illusory contours can be weakened or destroyed by disparity information inconsistent with occlusion.

Certain arrangements of inducers may support several different interpretations of occluding illusory figures, leading to ambiguous illusory figures (Bradley, 1987; Bradley \& Dumais, 1975; Scrivener, 1983; Shank \& Walker, 1989). Bradley and Dumais present a stimulus similar to that of Figure $17 \mathrm{a}$, which their subjects have reported perceiving as two different illusory figures: a six-pointed star and two triangles rotated $180^{\circ}$ with respect to one another, one lying atop the other. In addition, either of the two triangles in the latter percept can appear to be in front. At a particular time, the locations of the illusory contours depend on which figure and which depth ordering the observer is perceiving. The figure may spontaneously reverse, leading to a different set of illusory contours. A similar phenomenon is observed with spontaneously splitting figures, an example of which is depicted in Figure $17 \mathrm{~b}$ (Kanizsa,
1979; Kellman \& Shipley, 1991; Petter, 1956). Illusory contours are perceived along the boundaries of the shape that is perceived to be nearer to the observer, with only amodal completion along the borders of the further shape-a perception that spontaneously reverses with time. These effects, along with related phenomena in the areas of perceptual set and memory, indicate that perceived stratification can have a profound influence on the appearance of illusory contours.

Inducer completeness. The incompleteness of the inducing elements in edge-induced figures has long been held to be a contributing, if not the governing, factor in illusory contour formation. An incomplete inducer is a cue that there may be an occluding figure. Kanizsa (1955) presented a display consisting of four inducers shaped like crosses (Figure 18a) to demonstrate the ineffectiveness of complete (in a gestalt sense) stimuli in generating illusory contours. However, employing the same cross inducers, along with other inducers whose symmetry would be broken if amodally completed behind the illusory figure (as in Figure 18b), Day and Kasperczyk (1983) showed that illusory contours were generated by these perceptually complete inducers, albeit with less strength than for traditional incomplete inducers. This finding has been confirmed by Purghé and his colleagues, in a series of thorough examinations of the effects of completeness and symmetry on contour strength (Purghé, 1988, 1991; Purghé \& Coren, 1992a; Purghé \& Katsaras, 1991). In a similar vein, Rock and Anson (1979) showed that the perceptual set of the observers affected whether they perceived illusory contours with complete, but asymmetric, inducers (as in Figure 18c).

The concept of completeness cannot easily be defined in simple geometric terms. It certainly has relations to symmetry, smoothness, and regularity, but one must also take into account familiarity of the figure, perceptual set, and a number of other abstract factors. It is indeed a gestalt property. Thus, the apparent effect of inducer complete- a

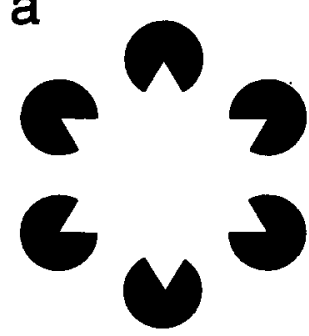

b

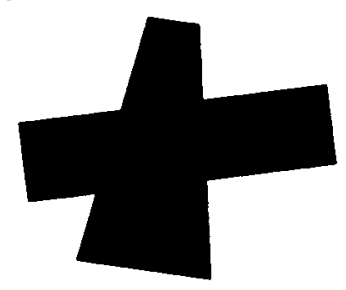

Figure 17. Some stimuli can induce "ambiguous" illusory contours which vary depending upon the perceived depth ordering of the inducers and illusory figures. (a) Bradley and Dumais's (1975) stimulus can support three percepts: a six-pointed star and two layering schemes of a pair of triangles oriented $180^{\circ}$ with respect to one another. From "Ambiguous Cognitive Contours," by D. R. Bradley and S. T. Dumais, 1975, Nature, 257, pp. 582-584 (Figure 3). Copyright 1975 by Macmillan Magazines Limited. Adapted with permission. (b) The positions of illusory contours in a "spontaneously splitting figure" (Kellman \& Shipley, 1991) depend on which shape is perceived to be nearer to the observer. 
a
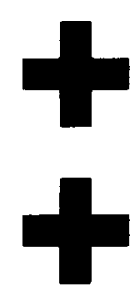

b
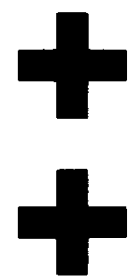
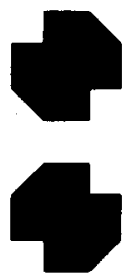
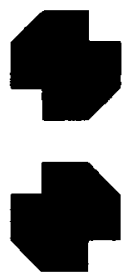

C

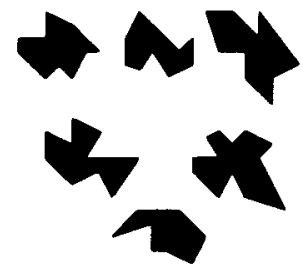

Figure 18. Perceptually complete inducers can still generate illusory contours, albeit with lesser strength than those that result from perceptually incomplete inducers. Figure 18a is from "Margini Quasi-Percettivi in Campi con Stimulazione Omogenea," by G. Kanizsa, 1955, Rivista di Psicologia, 49, pp. 7-30 (Figure 21). Copyright 1955 by Giunti Gruppo Editoriale. Adapted by permission. Figure $18 \mathrm{~b}$ is from "Amodal Completion as a Basis for Illusory Contours," by R. H. Day and R. T. Kasperczyk, 1983, Perception \& Psychophysics, 33, pp. 355 - 364 (Figure 3). Copyright 1983 by Psychonomic Society, Inc. Adapted by permission. Figure $18 \mathrm{c}$ is from "Subjective Contours and Apparent Depth," by S. Curen, 1972, Psychological Review, 79, pp. 359-367 (Figure 8c). Copyright 1972 by the American Psychological Association. Adapted by permission.

ness on illusory contour clarity is difficult to explain in low-level terms. Van Tuijl and Leeuwenberg (1982) have presented a language coding method that mathematically captures some elements of inducer completeness, but that is incapable of emulating many of the subtler aspects. Albert (1993) has pointed out that many stimuli employed in demonstrating the lack of illusory contours with complete inducers share a single geometric property-namely, a nearby edge parallel to the supporting edge, as in Figure $18 \mathrm{a}$. Further, Albert has shown that in many of these cases, tilting the offending edge away from parallel restores illusory contour strength while maintaining the perceptual completeness of the inducing elements. Sajda and Finkel (1995) have made similar claims about the ability of local mechanisms to emulate gestalt properties. Although these observations certainly do not explain the effects of inducer completeness, they represent examples of how simple determinants can give rise to seemingly complex phenomena.

The gestalt tendency of continuation has been related to completeness by several researchers (Kanizsa, 1974; Minguzzi, 1987). Minguzzi points out that inducing elements tend to continue (amodally) behind illusory figures. The nonsupporting edges of edge inducers continue in their curvilinear paths (e.g., circles missing sectors become amodally completed circles), while the lines of line-end inducers amodally extend through the illusory figure. Although completion requires knowledge of the overall inducer structure, continuation is a local tendency and thus is more readily definable in low-level terms. However, Davi, Pinna, and Sambin (1992) have found that there is no correlation between the perceived continuation of lines and either illusory contour strength or illusory figure brightness.

Perceptual set, learning, and memory. Rock and Anson (1979) demonstrated that the mental state of subjects had a significant effect on whether or not they perceived illusory contours. By pictorially cuing subjects to such abstractions as incompleteness and alignment - con- cepts typically associated with determination of the effectiveness of illusory contour inducers - they were able to markedly increase the percentage of observers who perceived an illusory figure in stimuli like those of Figure 18c. Similarly, Coren, Porac, and Theodor (1986, 1987) have shown that perceptual set may also have an effect on the shape of the perceived illusory figure. By cuing subjects as to what type of illusory shape to expect in an Ehrenstein figure (circle or square) they could bias the distribution of reported percepts. Indeed, even in the vision research community, many common stimuli that yield illusory contours, such as Ehrenstein and Koffka figures, were not recognized as such until many years after their initial presentations (Gregory, 1987; Kanizsa, 1955; Landauer, 1978).

Altering subjects' perceptual set may cause them to perceive illusory contours where they previously observed none, implying that learning may be involved in illusory contour perception. Gellatly (1982) provided subjects with stereoscopic cues that the triangle in a Kanizsa configuration was behind the inducers, giving rise to the "porthole effect" wherein subjects have the impression of seeing a triangle through three circular apertures. After viewing several of these stimuli, subjects reported having the same percept in the monocular domain with a standard Kanizsa triangle. Gellatly and Bishop (1987) demonstrated a similar example in which motion was used to cue an illusory contour which was then perceived in a static environment, and verbal explanations by Bradley and Petry (1977) were enough to cause subjects to perceive alternate illusory contours in an illusory Necker cube. In all these cases, subjects were provided with additional information about the relative depths of the illusory figure and the inducing elements. Previously unperceived contours became visible once the subjects were aware of the stratification of the inducers and the illusory figure. Wallach and Slaughter (1988), studying the role of memory in illusory 
contour formation, found that subjects more readily perceived illusory shapes familiar from previous experimental presentation than they did novel shapes. In all of these cases, the stimuli maintain a potential for illusory contour formation-all low-level determinants are consistent with contour generation--which is only realized when subjects are cued to the existence of the contours. In stimuli that support multiple illusory figures, the degree to which the inducing elements subserving each figure are compatible with low-level determinants may determine which illusory figure will be perceived. For example, in a Kanizsa configuration, a triangle is observed more often than three portholes, perhaps because the triangle inducers (the edges along the missing sector of the circle) are well aligned, whereas the circular porthole inducers (the curved, outside edges of the circle) require curved completion for subjects to perceive an illusory contour.

Attentional effects. Closely related to perceptual set are the effects that attention can have on the formation of illusory contours. In a visual search paradigm, Grabowecky and Treisman (1989) have shown that detection of an edge-induced illusory triangle is influenced by the number of background distractors, inferring from this that illusory contour perception is not a preattentive process. In the context of a texture segmentation task, Meyer and Fish (1987) have reported similar findings. Comparing the times required to discriminate between different shapes of edge-induced illusory forms and between equivalent outlined forms, Pritchard and Warm (1983) found that reaction times increased more for illusory form discrimination than for outlined form discrimination as attentional loads were increased, implying a greater attentional demand for illusory contour perception. The high attentional demand indicated by these experiments has been used to argue for a cognitive theory of illusory contour formation. However, Gurnsey, Humphrey, and Kapitan (1992) have pointed out that edge type illusory figures were employed in all of these studies, and they reason that the attentional findings may be related to the low contrast between illusory figure and background, as opposed to the high contrast between the inducers and background. Eliminating contrast and other interfering factors by using fields of lines with lineend-induced illusory contours, Gurnsey et al. (1992) have demonstrated parallel search and quick texture segmentation. Nevertheless, the earlier experiments highlighted differences in the saliency of real and illusory contours.

\section{Ecology of Determinants}

Given that illusory contour perception developed as a method of disambiguating low-contrast figures - to discover the most probable organization that would account for the visual stimuli-high-level determinants are particularly easy to relate to visual ecology. If, given our ecological problem, we were told to write down the features that should be important for determining the presence of illusory contours, it is likely that we would come up with all the high-level determinants discussed in this review. Depth information in conflict with illusory form stratification should weaken or destroy the proposed contour.
Complete inducers should be less likely to induce illusory forms than should incomplete inducers. Familiar shapes should be preferred to novel shapes. Every high-level determinant has a clear ecological purpose, easily understandable from the problem-solving perspective.

Some low-level determinants would doubtless find their way to our hypothetical list as well, but prescience would be necessary to include them all, since some determinants appear unrelated to, or even incompatible with, the task of reliably creating illusory forms. The reasons for the effect of inducer spatial extent and proximity are obvious enoughthe more the support, the more probable the existence of an occluding object. If objects generally have smooth, continuous edges, then the preference for aligned inducer edges becomes apparent. Anchors reflect the need to grasp at any information that can disambiguate contour paths left ambiguous by insufficient inducer support. Figural interferences, such as the outlining of an edge inducer, indicate incompatibility with occluding objects. Small-scale structure of an inducer, particularly a line-end inducer, may hint at a natural termination rather than an occlusion. But why should dots near an inducer weaken an illusory contour? Why should increasing line density eventually result in a decrease in contour strength? Why shouldn't support ratio be an absolute predictor of contour strength? It is unclear whether these determinants are epiphenomena of other ecological processes - unavoidable anomalies caused by the integration of the illusory contour problem with all the other problems of vision within a constrained neurophysiological framework - or represent some time-tested kernels of ecological truth. In either case, it is possible that perceptual groupings of proximal or otherwise spatially related elements, of both the inducing and the interfering variety, play a significant role in determining illusory contour strength.

\section{ILLUSORY CONTOURS AS EARLY VISION PHENOMENA}

A great deal of evidence suggests that illusory contours are initially formed early in the visual processing stream, although higher level processes help to determine the final positions and strengths of these preliminary contours. The primary evidence for early formation comes from a recent series of neurophysiological studies in which cells responding to illusory contours were found, but there is also a convincing body of supporting psychophysical evidence. By demonstrating an equivalence between real and illusory contours in sustaining visual illusions such as tilt and motion aftereffects, psychophysical studies have suggested that illusory contours obtain a neurophysiological reality at early levels of visual processing. Overviews of the relevant neurophysiological and psychophysical findings are provided below.

\section{Neurophysiological Evidence of Illusory Contours}

von der Heydt, Peterhans, and Baumgartner (1984).

In an influential paper, von der Heydt et al. (1984) presented results from single cell recordings indicating they 
had discovered neural correlates of illusory contours in V2 (but not V1) of macaque monkeys. Subsequent work in the same laboratory extended these original findings, not only establishing the existence of "illusory contour cells," but also providing data on the effect of a variety of parametric stimulus variations on cellular activity (Peterhans \& von der Heydt, 1989, 1991; Peterhans, von der Heydt, \& Baumgartner, 1986; von der Heydt \& Peterhans, 1989a, $1989 \mathrm{~b}$ ). In all these studies, stimuli consisting of abutting line gratings (Figure 19a) and stimuli consisting of two parallel bars with rectangular notches cut out (Figure 19b) were tested extensively. These two cases will be examined independently below.

Almost half of the cells examined in V2 by von der Heydt et al. (1984) exhibited sizable responses to drifting bars or edges and the illusory contour induced by drifting line gratings. For line gratings, they observed cellular response when the lines of the grating were oriented perpendicularly to preferred orientation of the cell; the cell was not merely responding to the inducing lines. The orientational tuning curve of a given cell, as determined with the illusory contour stimulus, closely resembled that cell's tuning curve as determined with a luminance-defined bar. Response was optimal when the lines in the grating were perpendicular to the illusory contours, monotonically decreasing as the lines were slanted away from perpendicular. Cells were not simply responding to individual line ends, however; the typical cell would not respond to a grating with only two or three bars, but it responded more and more strongly as additional bars were added, until a saturated level of activity was reached.

For the most part, the cellular recordings of von der Heydt et al. (1984) revealed cells whose responses to variations in inducer configurations closely resembled human psychophysical responses to those same variations - for example, increasing to a saturated response as the number of inducing lines increased. However, when the researchers shifted each half of the grating along the inducing lines so that the lines meshed or left a gap, many cells still responded strongly, especially in the case of a gap. This marks a divergence from human psychophysical data, because a

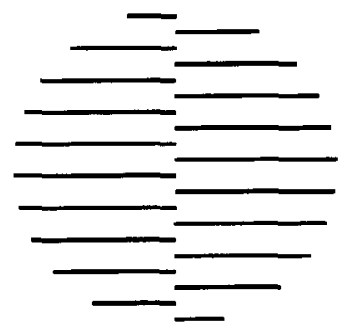

b

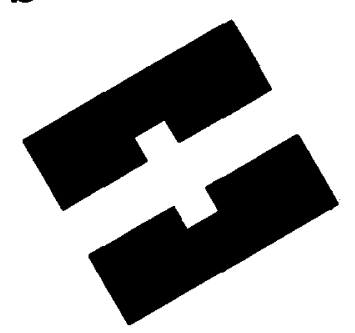

Figure 19. The stimuli employed by von der Heydt et al. (1984) in their neurophysiological experiments. From "Illusory Contours and Cortical Neuron Responses," by R. von der Heydt, E. Peterhans, and G. Baumgartner, 1984, Science, 224 (June 15), pp. 1260 1262 (Figure 2). Copyright 1984 by the American Association for the Advancement of Science. Adapted with permission. humans are extremely sensitive to the alignment of the line ends: illusory contours are destroyed with very little grating shift in either direction. Soriano et al. (1994) have reported that an overlap or gap of as little as a tenth of a degree markedly reduces the probability that subjects will report seeing an illusory contour.

As with line gratings, a significant fraction of $\mathrm{V} 2$ cells $(\sim 30 \%)$ responded well to notch type stimuli that yield illusory contours in humans (Figure 19b), although response levels were typically lower than for the line gratings. Cellular activity fell off with increasing notch separation and was greatly decreased when only a single notch was presented. Placing a very thin bar over the mouth of one of the notches significantly attenuated response levels, a finding perhaps related to figural interference effects observed psychophysically.

The neurophysiological responses to notch stimuli also parallel human psychophysical data. Cells that responded to the notch stimuli also tended to respond to the phaseshifted grating stimuli, as well as to real bars and edges. This finding led von der Heydt et al. (1984) to conclude that they had found a neural correlate of illusory contours. Further, since these cells responded to both real and illusory stimuli, they believed that they had discovered a unified boundary representation. Their findings led them to propose a model of illusory contour formation, discussed in the neural modeling section, which serves as the foundation for several more advanced architectures.

Redies, Crook, and Creutzfeld (1986). Employing phase-shifted gratings similar to those used by von der Heydt et al. (1984) (Figure 19a), Redies et al. (1986) studied the response of cells in visual areas 17 and 18 of cats. The researchers found that no simple cells in areas 17 or 18 responded to the illusory contours of the stimuli, but that some complex cells in both areas responded to the illusory contours. Redies et al. posit that the difference in areas of illusory contour cell location from those of von der Heydt et al. may well be due to a variation across species. Careful controls were employed to eliminate the possibility that cells were merely responding to the ends of individual lines, ensuring that some cells were indeed responding to a nonluminance-defined border-something at least similar to an illusory contour. Mean complex cell responses dropped off with increased line spacing, as was observed by von der Heydt et al.

The experiment of Redies et al. (1986) was ahead of its time; whether cats could actually perceive illusory contours was not known until 2 years later, when Bravo, Blake, and Morrison (1988) employed a discrimination task to determine that cats could indeed perceive Kanizsa figures. Soon after, de Weerd, Vandenbusche, de Bruyn, and Orban (1990) performed similar discrimination experiments with phase-shifted gratings. Employing Kanizsa figures rather than gratings, Gregory (1987) failed to find cellular responses in cat visual cortex; but his studies were limited to area 17 , and he made no effort to locate complex cells. Thus, whether edge-induced illusory contours can induce activity for cells within area 18 of the cat (or area 17 complex cells) remains unknown. 
Grosof, Shapley, and Hawken (1993). Grosof et al. (1993) have recently produced data indicating the existence of neurons in $\mathrm{Vl}$ of macaques that apparently respond to line-end stimuli similar to those employed by Redies et al. (1986). Consistent responses were observed in response to phase-offset sinusoidal gratings, with controls to ensure that cells were not responding to local edges. Cells exhibiting responses to the contour tended to be complex, but some simple cells with this characteristic were also discovered. Phase-offset sinusoid gratings give rise to illusory contours with support ratios approaching 1.0 ; there is real luminance contrast at almost every location along the contour, although the direction and strength of contrast varies periodically along the contour. Grosof et al. did find one Vl complex cell that also responded to more conventional line-end gratings with low support ratio, but the discovery of a single cell does not constitute convincing evidence of a mechanism for illusory contour formation.

Redies et al. (1986) and Grosof et al. (1993) found only cells that responded to very simple illusory contours with perfectly aligned line ends. As yet, there is no evidence that these neurons would respond to edge-induced illusory contours or curved illusory contours. These neurons may not be signaling illusory contours per se but may represent only the early stages of processing - a simple summation of aligned end-stopped cells. To establish the role that these neurons play in illusory contour perception, additional parametric studies of inducing stimuli structure must be undertaken-of the effect of grating overlap and gap, response to edge inducers, and response to curved illusory contours.

\section{Psychophysical Similarities Between Real and Illusory Contours}

In the debate between bottom-up and top-down proponents of illusory contour formation, psychophysical similarities and dissimilarities between illusory contours and real (luminance discontinuity) contours have played an important, though somewhat tiresome, role. Although the results of these similarity studies are conclusive in determining how real and illusory contours are related on given tasks, the experiments have had scant success in altering the views of proponents for either ideology. Many of the illusions or phenomena employed in these experiments are not well enough understood in themselves for the phenomena to be pinned down to specific levels of visual processing; thus, the establishment of a correlation between the response to two types of contours specifies only that illusory contours and real contours have the same perceptual significance at some unspecified level. Slightly more informative is the case of dissimilarity, in which the implication (by cognitivists at least) is that illusory contours gain perceptual significance after (at a higher level than) real contours.

For the purposes of determining the point of illusory contour generation within the visual processing stream, the most salient of the comparisons are those which deal with phenomena that have known neural correlates. Experiments indicating similarities between real and illusory contours in higher level characteristics such as reversibility of ambiguous figures (Shank \& Walker, 1989), texture rarefaction (Bressan, 1987), facilitation of amodal completion behind a figure (Bruno \& Gerbino, 1987), and figureground organization (Peterson \& Gibson, 1994) are necessary, serving to support the intuitive sense that illusory figures have the same cognitive significance as real figures, particularly with respect to stratification in depth, but such experiments do not provide much assistance in pinpointing the locus (or loci) of illusory contour formation. The large number of comparative studies precludes all but the briefest mention of most: Only comparisons with profound theoretical implications will be discussed here in detail.

Orientation and motion estimations. Some of the earliest and most influential experiments demonstrating perceptual equivalence between real and illusory contours involved the examination of biases in motion and orientation estimations. By revealing the similarities between real and illusory contours in the realms of tilt aftereffects (Smith \& Over, 1975, 1976) and motion aftereffects (Smith \& Over, 1979; Weisstein et al., 1977), early researchers made a compelling case for a similar representation of the two contour types. These aftereffects are often attributed to short-term habituation in early visual cortex (Barlow \& Hill, 1963; Movshon, Chambers, \& Blakemore, 1972), rendering the observed similarities between real and illusory contours theoretically important. In further implication of a shared internal representation, all of these aftereffects appear to cross over between real and illusory contours, a finding supported by more sophisticated recent studies on tilt aftereffects (Berkley et al., 1994; Paradiso et al., 1989). Smith and Over (1977) have also demonstrated similarities in orientation masking, with full crossover between contour types.

Vogels and Orban (1987) discovered equivalent biases in estimation of real and illusory contour orientation, but with an asymmetric learning effect in which practice on illusory contour orientation estimation carried over to real contour estimation, but not vice versa. A number of researchers have employed illusory contour versions of classical illusions involving impaired estimations of orientations. It appears that illusory contours facilitate the Bourdon illusion (Walker \& Shank, 1987, 1988a, 1988b; Wenderoth, Criss, \& van der Zwan, 1990) but slightly decrease the effect of the Poggendorff illusion (Day, Dickinson, \& Jory, 1977; Goldstein \& Weintraub, 1972; Meyer \& Garges, 1979). General equivalence in orientation estimation is a strong, though not unequivocal, argument for early representation of illusory contours.

Time course studies. Whether the perception of illusory contours parallels the perception of real contours in terms of the saliency of the contour as a function of time is an important theoretical issue. Cognitive theories generally predict a significant lag between detection of illusory contours and detection of real luminance gradients; inducer information must make its way to higher levels of the brain, cognitive contour formation must occur, and (perhaps) illusory contour information must make its way back down to earlier levels of the visual cortex. However, this may also be true for neural models that incorporate feed- 
back loops. Employing a Kanizsa figure, Reynolds (1981) showed that perception of illusory contours (under masking conditions) was not reliably reported unless the inducing stimuli were presented for longer than $75 \mathrm{msec}$, although the inducing elements were clearly perceived at $50 \mathrm{msec}$. In a second experiment, a Kanizsa figure with overlaying bricklike texture did not yield a salient contour with extended viewing, presumably because of the figural interference of the intersecting lines. When flashed, however, a contour was perceived with durations between 75 and $250 \mathrm{msec}$; but it was not perceived for durations below or above this range, perhaps indicating an initial top-down hypothesis rejected upon further consideration. A third experiment, in which the texture was seen as transparent when observed in steady state (much as in Rock \& Anson, 1979), showed a similar effect, with contours becoming evident again after $400 \mathrm{msec}$ - again compatible with a hypothesis process (for further details, see the discussion of cognitive theories and the related figure in the section Phenomenological Models of Illusory Contour Formation).

Reynolds's (1981) results differ markedly from those of Gellatly (1980), who employed a similar method to study time course saliency. Gellatly found that the illusory triangles became apparent after $10 \mathrm{msec}$, well before the inducers themselves were perceived. The variation in results undoubtedly arises from the difference in masks used. Whereas Reynolds used noisy, solid circles covering the inducers, Gellatly employed rings around the inducers, thus employing the metacontrast paradigm along the outer inducer edges but no masking along the illusory contour supporting edges (edges of the missing sector). The significant effect of the mask is confirmed by the use of an inducer outline as the mask. Gellatly demonstrated that such a mask increased the duration necessary for one to perceive the contour to several hundred milliseconds.

In a study of visual persistence, Meyer and Ming (1988) note that illusory contour perception appears to lag behind the perception of real contours, while also observing that illusory contours persist much longer than their inducing elements, once the latter have been replaced by blank fields. Petry and Gannon (1987) had subjects rate brightness as a function of time as masked figures were flashed, finding that illusory figure brightness lags behind real figure brightness but exhibits the same general time course plot. Besides these studies, a number of anecdotal confirmations of the delay in illusory contour formation have been provided by researchers working with apparent motion (Mather, 1988; Petersik, 1987; Ramachandran, 1986a; Vallortigara, 1987; von Grünau, 1979). In these studies, stimulus presentation times had to be significantly longer for illusory figures than for real figures in order to achieve the same degree of apparent motion. Of course, the support of apparent motion itself is an important indicator of equivalence between the contour types.

Time course studies clearly indicate that the substrate of illusory contour formation cannot consist solely of bottom-up neural processing. However, the delays in contour formation are not so lengthy as to be compatible only with cognitive approaches to illusory contour perception.
Such delays would also be expected in neural systems that rely on feedback to establish illusory contours. To explain Reynolds's (1981) intriguing results at a neural level requires more sophisticated modeling techniques, but his data are not incompatible with recent designs which incorporate stratification and depth effects (Grossberg, 1994; Sajda \& Finkel, 1995).

Binocular rivalry. Bradley (1982) has examined the effect of binocular rivalry on Kanizsa triangles. When a normal illusory triangle was presented to one eye and an inverted illusory triangle to the other, a stimulus closely related to Figure 17a, subjects tended to perceive a fusion of the two triangles into a six-pointed star which would occasionally split into two overlying triangles in the manner observed for other ambiguous illusory contours. Conversely, if the illusory triangles were replaced by real triangles of slightly higher luminance than that of the background, binocular rivalry occurred, and the six-pointed star, if observable at all, was highly unstable. Fahle and Palm (1991), however, observed binocular rivalry between illusory contours with dichoptic stimuli presentation, albeit less complete than observed between real contours.

Other comparisons. In studying flicker haloesstreaky bands surrounding stimulus edges when the stimulus was presented intermittently at frequencies below the fusion frequency-Remole et al. (1985) noted strikingly similar halo extents for real and illusory contours as both the flicker frequency and stimuli luminances were changed. This experiment supports a neural equivalence of contour types, since flicker haloes are thought to have an early locus (Remole, 1970). Arguing against early instantiation, Halpern and Warm $(1980,1984)$ studied image fragmentation - the disappearance and reappearance of parts of the stimuli at extended viewing durations - and found that illusory figures fragment far less frequently than their luminance-defined counterparts, although the inducing elements fragment more frequently. Since fragmentation is commonly linked to neural adaptation and fatigue (MacKinnon, Forde, \& Piggins, 1969), Halpern and Warm's data preclude illusory contours from appearing at the same neural level at which this adaptation is occurring. Since adaptation may be occurring very early in the visual processing stream, however, these fragmentation results are incompatible only with the simplest of bottom-up models.

\section{Developmental and Clinical Findings}

Several studies of the development of illusory contour perception in children have been performed in the last decade. Bertenthal, Campos, and Haith (1980), who studied the response of infants to Kanizsa-type displays, found a marked change in detection accuracy between the ages of 5 and 7 months. Although the authors argued that the infant was indeed seeing the illusory contour, they admitted that the infants might have been responding to some simple figural effect such as local brightness contrast. Soubitez (1982) noted that children did not tend to perceive the central portion of an Ehrenstein figure brighter than that of an Ehrenstein figure with a real circle along the inner line ends, an addition known to reduce bright- 
ness for adults (Ehrenstein, 1941), until 3 years of age. Abravanel (1982) found that likelihood of perception and recognition of Kanizsa-like illusory figures tended to increase with age, with a significant change from ages 3 to 5 years. All the 3- to 4-year-olds tested in an informal study by Kennedy (1987) reported seeing what he classified as illusory contours. In all these cases, communication problems inherent in dealing with young children may have had a significant effect, so it is difficult to determine the reliability of these studies. However, one cannot rule out the possibility that perception of illusory contours does not develop until several years after birth, to some degree representing a learned skill. If this proves to be the case, learning at the ages mentioned above would correspond to changes in cognitive perceptual strategies, as opposed to changes in the mechanisms of the lower levels of the visual cortex. This would, of course, be a strong argument for an increased role of higher level factors in contour formation.

Two important clinical studies have been performed in order to establish correlations between certain visual deficits and illusory contours. Hamsher (1978) studied the link between scores on benchmark stereo performance tests and Kanizsa-like illusory contour perception for brain-damaged patients with a range of perceptual disorders. A strong correlation was found between the inability to perceive illusory contours and poor stereo performance, a similarity that Hamsher suggested was attributable to deficits in the discrimination of subtle difference in the spatial location of features. If this is indeed the common factor, Hamsher's experiment underscores the deleterious impact of both inducer misalignment and small-scale interference on contour formation. Stevens (1983) studied a single patient who had trouble interpreting interpositions, finding that although this patient could not see illusory contours monocularly, stereoscopically presented illusory contours were seen vividly. This study suggests that, at least for some stimuli, salient illusory contours require that subjects perceive illusory figures as being separated in depth from the inducing elements.

\section{PHENOMENOLOGICAL MODELS OF ILLUSORY CONTOUR FORMATION}

The first two phenomenological models of illusory contour formation set the distinctly different paths that research for the following decades was to take. Ehrenstein (1941), in a highly influential study of variations of his eponymous figures, was primarily interested in the nature and origin of illusory brightness. Future researchers along this line, whom I will refer to as members of the brightness camp, expanded upon Ehrenstein's theory and expounded that illusory brightness actually drove illusory contour formation. On the other hand, Kanizsa (1955) stressed the importance of understanding illusory forms as a whole, downplaying the significance of such specific characteristics as brightness and clarity. These features, he believed, followed naturally from the instantiation of an illusory form. The researchers concentrating on the form of illusory figures I will refer to as members of the form camp.
The two paradigms differ markedly not only with respect to which aspects of the illusory figure they emphasize, but also with respect to the level of abstraction from which they must be approached. Those who are in the brightness camp assume that illusory brightening drives illusory contour formation, and thus they must attempt to explain in neurophysiological terms whence the brightening derives. Those who are in the form camp argue that the task is to explain what particular geometrical aspect of the stimuli causes a viewer to segment the scene so as to give rise to an illusory figure. In the earliest days of illusory contour research, the two approaches could safely be classified as low-level and high-level respectively, but this distinction is no longer valid. Although the initial concepts of neural modeling of contour formation did indeed arise within the brightness camp, more recent models have recognized the importance of factors previously stressed only by members of the form camp - specifically, the role of cues to depth in illusory contour formation. Below, I sketch a history of the two ideologies: the evolution of the form camp theories from the phenomenological to the concrete, and the gradual relegation of brightness to the role of a secondary process. The neural models of contour formation, which give all indications of representing the future of illusory contour modeling, are reviewed in a subsequent section.

\section{Brightness Theories}

Although the progenitor of brightness theories of illusory contour formation, Ehrenstein's (1941) work does not technically fall into the category of illusory contour research. He was primarily interested in explaining the striking brightness effects that he noted in the Ehrenstein figure, making no attempt to link illusory brightening to illusory contours. Indeed, it is not obvious that Ehrenstein even noticed the illusory contours within his stimuli. In his brightness research, however, he established many of the key configural parameters that would later be used to study contour clarity, as well as brightness, including number of inducing lines, width of lines, size of stimuli, and small-scale shape of the line ends. Ehrenstein's explanation for illusory brightening was that the inducing elements were laterally blurred into their surround because of eye movements, resulting in a real contrast difference between the central region and the surround. This theory was discredited by Spillman, Fuld, and Gerrits (1976), who observed illusory brightening when an Ehrenstein figure was flashed for a duration brief enough to preclude eye movements, but Ehrenstein's work stands as a concise examination of the parameters that affect illusory brightening and illusory contour strength.

\section{Gestalt Theories}

Kanizsa (1955) presented an impressive array of stimuli containing illusory contours, of both the edge and the line-end type. Prior to Kanizsa's paper, most examples of illusory contours had been rather weak, consisting primarily of straight contours, like those in Schumann (1900, 1904). Kanizsa, a member of the Gestalt school, saw illusory figures as a consequence of the "poor form" of the in- 
ducers. That is, he saw the inducers as incomplete in the gestalt sense. He posited that in a configuration such as that of Figure 2a, the incomplete circular inducers are reorganized by the perceptual system so that they become more complete or regular circles, because there is a tendency in visual perception toward simplicity and stability: prägnanz. Reconciliation of this percept with the missing inducer sectors requires the perception of an illusory square occluding parts of each circle. Note that Kanizsa considered neither the completion nor the reconciliation to be a conscious decision, but rather an autonomous, selfregulating process. The concept of completion of partially occluded objects was not new, but the idea that such amodal completion (of the inducers) could occur in the absence of real (luminance induced) occlusion and thus be intimately related to illusory contour formation, certainly was. Kanizsa noted that the brightness theory was a possible alternative, but he discounted it primarily on the grounds that illusory figures appear brighter than they should if conventional brightness contrast alone was responsible for the brightening. This argument has been validated experimentally in studies showing brightness to be greater in illusory figures than in controls having the same local configurations (Bradley \& Mates, 1985; Coren \& Theodor, 1975; Dresp, 1992).

Aspects of the gestalt approach to illusory contour formation have not held up well to scrutiny. The basic tenet of this approach is that the (amodal) completion of irregular inducing elements is primarily responsible for driving the formation of illusory figures. Therefore the existence of inducers that are already regular (in the gestalt sense), or do not become more regular (or even become more irregular) upon completion, would refute such a theory. $\mathrm{A}$ number of researchers have demonstrated stimuli with such inducers (Davi et al., 1992; Day \& Kasperczyk, 1983; Kellman \& Shipley, 1991; Purghé, 1988, 1991; Purghé \& Coren, 1992a; Purghé \& Katsaras, 1991), several examples of which are depicted in Figure 20. Kanizsa himself retracted his original explanation of illusory contours formation (Kanizsa, 1976, 1987), but there can be little doubt that regularity of the inducers can have a significant effect on the strength of the resulting contour (see discussion of high-level determinants). In a related area, Wasserstein, Zappulla, Rosen, and Gerstman (1987) have shown a linkage between poor closure ability and lack of illusory contour perception in brain-damaged subjects. The gestalt characteristic of continuation, which has obvious bonds with both completion and closure, has also been implicated as a driving force behind contour formation (Kanizsa, 1974; Minguzzi, 1987). As is the case with completion, however, psychophysical experiments have not supported a causal link between inducer continuation and contour strength (Davi et al., 1992).

\section{Depth Theories}

Chronologically, the next major explanatory initiative regarding illusory contours came again from the form camp, as Coren (1972) proposed his "depth cue" theory. Noting that all illusory figures (known at that time) were perceived as occluding the inducers, he posited that interposition acts as a cue for illusory contour formation. That is, once depth cues have been perceived, the visual system supplies appropriate surfaces to account for the apparent depth stratification of the stimuli. Stimuli that yield weak illusory contours do so because depth cues are weak. Whereas Kanizsa (1955) argued that completion of irregular inducers drove contour formation, Coren argued that the implicit depth cues signified by some aspects of these irregular inducers drove contour formation, which in turn drove amodal completion of the inducers behind the illusory figure.

Unfortunately, Coren failed to specify any concrete depth cues other than interposition, leaving himself open to the criticism of circularity of definition by Rock and Anson (1979): there can be no interposition before two surfaces are perceived, and in Coren's hypothesis, there will not be two surfaces until interposition is noted. Further- a

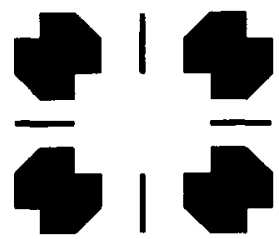

b

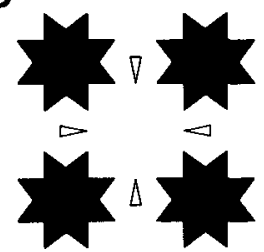

C

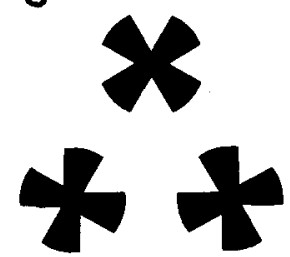

Figure 20. In contradiction with Gestaltist predictions, complete inducing stimuli can generate salient illusory contours, as indicated by these examples of (a) Day and Kasperczyk (1983a), (b) Purghé (1991), and (c) Kellman and Shipley (1991). Figure 20a is from "Amodal Completion as a Basis for Illusory Contours," by R. H. Day and R. T. Kasperczyk, 1983, Perception \& Psychophysics, 33, pp. 355-364 (Figure 3). Copyright 1983 by Psychonomic Society, Inc. Reprinted by permission. Figure $20 \mathrm{~b}$ is from "Is Amodal Completion Necessary for the Formation of Illusory Figures?" by F. Purghé, 1991, Perception, 20, pp. 623-626 (Figure 2). Copyright 1991 by Pion Limited, London. Adapted with permission. Figure $20 \mathrm{c}$ is from "A Theory of Visual Interpolation in Object Perception,” by P. J. Kellman and T. F. Shipley, 1991, Cognitive Psychology, 23, pp. 141-221 (Figure 41). Copyright 1991 by Academic Press. Reprinted with permission. 
more, not all illusory figures involve interposition, as is the case with diffuse illusory contours (Kennedy, 1976, $1978 \mathrm{~b}, 1979$ ), physically three-dimensional constructs (Ware \& Kennedy, 1977, 1978a, 1978b), stimuli with inducers on the inside of the illusory figure (Kennedy \& Lee, 1976; Parks, 1986; Purghé \& Coren, 1992a), and, indeed, even the phase-shifted grating first presented by Coren himself (Coren, 1972). Coren's formulation of the depth-cue theory is untenable, but, as I will point out later, his conjecture contains the kernel of what is now a widely accepted general theory of illusory contour formation.

\section{Cognitive Theories}

Gregory (1972) authored the next important paper from the form camp, arguing that illusory contour formation was largely a cognitive or cognitive-like process. Kanizsa and Coren likewise believed illusory contour formation to be a rather high-level operation, but both stressed the passive and automatic nature of the process. On the other hand, Gregory placed the emphasis on a postulation method wherein cognitive explanations of the stimuli configuration were proffered, presumably subconsciously. In a more comprehensive paper, Rock and Anson (1979) referred to illusory figures as solutions to a perceptual problem, theorizing that the solutions are arrived at through some cognitive process. In the case of a Kanizsa figure, for example, the percept of an occluding triangle of the same color as the background's is more likely (in some sense) than the veridical configuration of three incomplete circles with perfectly aligned sector edges. Van Tuijl and Leeuwenberg $(1979,1982)$ have attempted to quantize the likelihood of a given percept via an energy/information minimization approach. Using a language code for pattern representation, they have demonstrated a high degree of correlation between subjects' perceptions and the efficiency of a given perceptual organization.

In support of their arguments, Rock and Anson (1979) show the effect of perceptual set on contour perception (see earlier discussion). If a cognitive postulation process is responsible for instantiation of illusory contours, then altering cognitive expectations should (and does) alter contour perception. Another convincing experiment involves the superposition of a grating pattern over a Kanizsa figure, as described in the high-level determinants section and depicted in Figure 16 (Rock \& Anson, 1979). The perceived depth of the grating has a profound effect on whether subjects perceive an illusory figure or not. In studying the time course of illusory contour perception, Reynolds (1981) employed a similar (monocular) stimulus in which a regular pattern was superimposed on a Kanizsa figure, as described in the discussion of psychophysical similarities between contour types. The salience of the illusory contour varied as a function of time, apparently indicating time-varying cognitive hypotheses regarding the relative depths of the inducers, overlayed pattern, and illusory figure. Additional support for this form of cognitive theory comes from the perception of reversible and ambiguous illusory figures (Bradley, 1982, 1987; Bradley, Dumais, \& Petry, 1976), the effects of learn- ing and memory on contour perception (Coren et al., 1986, 1987; Gellatly, 1982; Gellatly \& Bishop, 1987; Landauer, 1978; Pritchard \& Warm, 1983; Wallach \& Slaughter, 1988), and other time course studies (Meyer \& Fish, 1987; Pomerantz, Goldberg, Golder, \& Tetewski, 1981; Takahashi, 1993).

In spite of a considerable degree of empirical support for certain aspects of the problem-solving approaches, these theories are no longer in favor as the primary explanation of illusory contour formation. To disprove cognitive theories absolutely would be extremely difficult, because of their relative lack of specificity in the computational sense of affording testable implementations or simulations. Given that problem-solving approaches are merely restatements of the ecological purpose of illusory contours, we would expect that psychophysical data would offer few conflicts with cognitive theories. However, the recent neurophysiological data of von der Heydt et al. $(1984,1989 b)$ and the similarities observed between real and illusory contours in terms of low-level perceptual effects indicate a relatively low-level instantiation of illusory contours. Although not completely incompatible with cognitive theories because of the extensive degree of topdown feedback to visual cortex (Zeki \& Shipp, 1988), these data strongly suggest that bottom-up mechanisms can themselves sustain the basic elements of illusory contour formation. Neural models indicate a range of possible incarnations that such mechanisms may take.

Although cognitive theories of illusory contour formation appear unparsimonious now that local, low-level features and processes sufficient to produce basic illusory contours have been identified, it would be a critical error to disregard the role that cognitive factors play in establishing the perception of these contours. Low-level neural models serve as strong foundations for illusory contour formation, but they cannot represent the entire process. Bottom-up models can never explain the profound effects that perceptual set, memory, and attention have on the course of illusory contour formation. In addition, illusory contours are not perceptually identical to real contours: we can often distinguish between the two types (Ware \& Kennedy, 1978a, 1978b), and they are not always equivalent in perceptual comparisons. Ultimately, the actual perception of illusory contours is not only a cognitive process, but doubtless a process that also manifests itself in lower level neural representations.

\section{Brightness Theories Revisited}

Brigner and Gallagher (1974) first examined the link between conventional brightness contrast and illusory contour brightness and strength. Recent studies have shown that the degree of illusory darkening in a Kanizsa square with light-on-dark inducing elements is proportional to the degree of conventional brightness contrast in similar control stimuli (which do not result in illusory figures) under variations of spatial and luminance characteristics of the inducing elements (Dresp, 1992; Dresp et al., 1990). Using flickering stimuli, Coren (1991) determined that figure brightness and contour strength were dependent on the level of lateral inhibition in the retina-inter- 
actions responsible for brightness contrast effects (Ratliff, 1965). Dresp (1992) has demonstrated an increased sensitivity in detection of small targets near the inducers of a Kanizsa square but not in the center of the illusory figure, implying that local brightness mechanisms may be responsible for generating illusory brightness but cannot be responsible for the filling in of the illusory figure that results in the perception of a form with homogeneous brightness. Although simultaneous contrast may explain illusory brightness effects (at least for edge inducers), proponents of this explanation for illusory contours failed to address a key issue: Namely, how are the actual illusory contours generated? It seems odd, in retrospect, how cavalier early researchers were about relegating contour clarity to secondary status. The typical explanation of the contours themselves was that once illusory brightness was established, the information was "then used together with physically present brightness gradients to generate perceptions" (Frisby \& Clatworthy, 1975) - a rather opaque exposition.

Another major problem with the conventional brightness contrast approach is that it fails to explain the striking brightening in figures containing very little contrast, such as the Ehrenstein figure. By slanting the inducing lines of an Ehrenstein figure, Kennedy (1978a) showed that more conventional contrast (due to inducers lying tangentially to the illusory circle) did not result in stronger illusory brightening and in fact weakened both clarity and brightness considerably. Frisby and Clatworthy (1975) addressed the issue of brightness in line-end induced figures, noting the existence of increased brightness at the ends of lines - a phenomenon later referred to as "brightness buttons" by Kennedy (1979). In revised brightness theories, these buttons play the same role as conventional brightness contrast, spreading out in some undefined way to form the illusory figure. Similar approaches were put forth by Kennedy (1978a, 1979, 1988) and Jory and Day (1979; Day \& Jory, 1980), the latter of whom introduced the widely used term dissimilation to capture the brightness button effect. Frisby and Clatworthy went so far as to propose crude receptive fields capable of generating dissimilation, a noteworthy predecessor to the neural models that would follow. Jory (1987) reported an increase in luminance sensitivity near the ends of lines, a finding bolstered by a recent comprehensive study by Dresp (1993). Dresp found that detection thresholds for light dots were lowered when they were in the proximity of the ends of light lines, but not for dots near "T" or "L" junctions, an indication that only in the absence of perpendicular elements do line ends generate brightness buttons. The dissimilation theories, which invariably included simultaneous brightness contrast effects as well, could explain most brightness effects in both edge and line-end illusory figures, but they suffered from the same lack of attention to actual contour formation as did conventional brightness theories.

\section{Spatial Frequency Theories}

During the debate between the brightness and form camps, a third party briefly entered the fray. Ginsburg $(1975,1987)$ and Becker and Knopp $(1978)$ proposed that stimuli inducing illusory contours actually contained the illusory form information in their spatial frequency spectrum - that the illusory forms were not, in fact, illusory if one considered only the low-frequency information of the inducer configuration. If the early stages of visual processing had various frequency bands, these low-frequency illusory forms could easily be perceived directly. This theory was discredited by Tyler (1977), who attacked Ginsburg's methodology; Parks and Pendergrass (1982), who showed the existence of illusory contours without lowfrequency correlates; and Parks (1983), who showed that existence of a low-frequency form did not necessarily yield an illusory form. Nevertheless, it is important that researchers, especially in neurophysiological studies and research involving animals and infants where it is difficult to ascertain exactly what the subjects are perceiving and to what they are responding, ensure that low spatial frequency content of displays does not play a significant role in what they believe to be illusory contour perception (Paradiso et al., 1989; Vogels \& Orban, 1987). Lack of such controls casts doubt on the reliability of many of the animal and infant studies of illusory contour perception.

\section{Multistage Theories}

Day and Jory (1980) proposed that illusory contour formation was a two-stage process, the first stage being the instantiation of illusory brightness via brightness contrast and dissimilation, and the second being the formation of the sharp illusory contour itself. Here, then, was explicit recognition from the brightness camp that contour formation itself was not a trivial, obvious process. However, no explicit method was proposed to explain contour formation. Halpern (1981) was one of the first to note that the solution to the problem of illusory contour formation would not come from one camp or the other, but rather from an amalgamation of different theories.

Soon after, theories considering illusory brightening as the primary factor in illusory contour formation received a fatal blow from the short paper by Prazdny (1983), who displayed figures similar to that in Figure 9a, consisting of equal numbers of dark and light inducers on a gray background which still yielded a salient boundary. Many similar qualitative displays have been demonstrated (Grossberg \& Mingolla, 1985a; Hershberger \& Stallard, 1984; Shapley \& Gordon, 1985, 1987), while Kellman and Loukides (1987) have confirmed with quantitative psychophysics that such inducing stimuli can indeed induce clear illusory contours in the absence of any illusory brightness.

Advancing interdisciplinary approach one step further, Day $(1986,1987)$ attempted to reconcile bottom-up and top-down theories. De-emphasizing the importance of dissimilation per se, Day conjectured that it was because line ends were an example of Coren's depth cues that they were important in illusory contour formation. A link between neurophysiological data - previously the stronghold of the brightness camp - and the form camp was thereby established. This theoretical vein has been extended by Kellman and Loukides (1987), who posited that spatiotemporal discontinuities - abrupt changes in object borders, 
positions, luminances, binocular depth, and so forth-are the most general of depth cues and therefore drive illusory contour formation. Shipley and Kellman (1990) have shown that contour formation is dependent on the presence of spatial discontinuities in the inducing stimuli, at least for inducers containing sharp luminance edges. Heitger, Rosenthaler, von der Heydt, Peterhans, and Kübler (1992) have since proposed a specific neural model for the determination of spatial discontinuities in the luminance domain. Examples of other spatiotemporal discontinuities that may produce illusory contours include jumps in binocular depth and accretion/deletion of background elements.

A basic, although generally unspoken, tenet of many cognitivists had been that low-level mechanisms were incapable of solving the ecological problem posed by illusory contour inducing stimuli. The recognition that depth cues can be assigned geometric definitions, the development of neural mechanisms to integrate these cues, and the strong neurophysiological and psychophysical evidence for early instantiation of illusory contours have combined to legitimize neural models of contour formation in the eyes of many researchers. Within the last 3 years, the number of such models has more than doubled, and significant enhancements have been made to the earlier systems. As I will show in the next section, however, the apparent resolution of the brightness-form conflict has not led to a less contentious field of research; there are fundamental differences in the approaches to neural modeling as well. In addition, there are still many unresolved issues regarding the integration of higher level determinants and cognitive factors into neural models.

\section{NEURAL MODELS OF ILLUSORY CONTOUR FORMATION}

Although neural systems represent the newest generation of models of illusory contour formation, they certainly do not lack in sophistication or complexity. These architectures consist of networks of cells with weighted connections between units, and, to varying degrees, they are meant to describe the structure as well as the function of the networks in the visual cortex that are responsible for illusory contour formation. Unlike most of the phenomenological models discussed in the previous section, neural models contain analogues of biological substrates as well as a theoretical basis of contour formation. Neural models were rejected out of hand by early researchers, because they believed such models were incapable of emulating even such relatively simple phenomena as curved illusory contours (Gregory, 1972). Early evaluations were made on the basis of ideas such as those of Jung and Spillman (1970), who proposed that line-end illusory contours might result from brightness induced by cells with elliptical receptive fields at the ends of lines (also Frisby \& Clatworthy, 1975), and Stadler and Dieker (1972), who proposed that illusory contours were the result of partial activation of the same feature detectors that were responsible for perception of luminance-defined contours. Indeed, many of these early model outlines were not specified well enough to truly explain the formation of any contoursstraight or curved-across extended regions of homogeneous luminance.

Ullman (1976) presented the first true computational model of illusory contour formation. His model is neural in the sense that it consists of several layers of locally interconnected cells. Based on completion collinear to edges and lines, Ullman's model consists of three layers of cells connected in such a way as to achieve a preference for straight-line completion, but with an allowance for curved completion when straight-line completion is not possible. These completion constraints are accomplished solely by local connections: cells excite neighbors that are roughly collinear and have similar orientations. Within the first two system layers, these local interactions establish a number of fuzzy curves. Competition in the third layer forces a choice of specific local curves. In the case of inducers that are not aligned collinearly, the illusory contours created by Ullman's model have minimal curvature, consisting of circular arcs tangent to the inducer edges. Although Ullman made no attempt to locate a particular neural substrate of his model, he showed that locally connected "neurons" could produce curved illusory contours.

Ullman's (1976) model pioneered neural models of illusory contour formation but received little attention from the research community, probably because (1) it only explained completion parallel to edges, ignoring the perpendicular completion of line-end inducers, (2) it was a computational vision model in a time of limited computer power, defying widespread simulation, and (3) it appeared to have little basis in real neural structures. It was not until the influential papers of Grossberg and Mingolla (1985a, 1985b) and Peterhans et al. (1986) that neural systems received widespread attention. These models had solid bases in physiology and could generate edge- and lineend-induced illusory contours, explaining the rudiments of both parallel and perpendicular completion.

The approach of Peterhans et al. (1986), which has inspired a variety of more sophisticated models (Finkel \& Edelman, 1989; Kellman \& Shipley, 1991), bases all illusory contour induction on line ends. Line-end (or discontinuity) models rest on the supposition that illusory contours are generated solely by ends of lines and the corners of edge inducers, with completion primarily in directions perpendicular to the inducing lines or corner elements. Although their classification might seem to limit their explanatory role to line-end inducers, these models still maintain the capability of generating illusory contours with edge inducers. Unlike the parallel completion approach of Ullman (1976) or the perpendicular completion approach of Peterhans et al., hybrid models encompass both forms of completion. More importantly, however, illusory contours may be generated by either line ends or edges in hybrid systems. Although they employ markedly different paradigms, both the boundary contour system of Grossberg and Mingolla (1985a, 1985b) and the newly proposed model of Heitger and von der Heydt (1993) include mechanisms for induction by both line ends and edges, along with parallel and perpendicular illusory contour completion. 


\section{The Line-End Models}

Peterhans et al. (1986). On the basis of their neurophysiological studies, Peterhans et al. (1986) proposed a model of illusory contour formation in which real and illusory contour representations are unified in early visual cortex (V2). Illusory contours are generated by endstopped cells whose receptive fields lie along a line roughly perpendicular to their preferred orientation, as depicted in Figure 21a. Pairs of these cells multiplicatively gate one another, ensuring that a single inducer cannot trigger formation of an illusory contour. The output of each gating is summed with all other gated outputs, as well as with the activity of complex cells whose positions and preferred orientations match the line along which the end-stopped cells lie. The gating of end-stopped cells signals the presence of illusory contours, while the complex cells signal the presence of real contours. A unified contour representation is achieved by summing these two representations.

The model of Peterhans et al. (1986) is based on perpendicular completion between line ends. How can edgeinduced illusory contours be explained in this context? Peterhans et al. posit that the corners of edge inducers trigger end-stopped cell activity, as depicted in Figure $21 \mathrm{~b}$. Since the same end-stopped cells that responded to line ends can be triggered by the corners of edge inducers, contour completion can occur with both inducer types in directions perpendicular to the preferred orientations of the individual end-stopped cells. This system introduces a paradigm common to many models of contour formation: the existence of separate pools of cells for real and illusory contour detection. Complex cells are employed for detection of real luminance gradients, whereas cells that respond to pairs of end-stopped cells are employed for detection of il- lusory contours (and for strengthening of degraded occlusions). This dichotomy, although shared by most other models of illusory contour formation, is not a requisite characteristic for such modeling, as has been shown by Grossberg and Mingolla (1985a, 1985b, 1987a, 1987b).

Finkel and Edelman (1989). The system of Finkel and Edelman (1989) is founded on the preliminary work of Peterhans et al. (1986), but it represents a more sophisticated model, with significantly greater explanatory power. Whereas Peterhans et al. only attempted to explain the simplest of illusory contour phenomena, Finkel and Edelman have explored subtle issues such as the integration of contour signals from different modalities and figural interference between real and illusory contours. However, as in the Peterhans et al. model, end-stopped cell activity drives all illusory contour formation. A diagram of the occlusion modules of Finkel and Edelman's complex model is presented in Figure 22. The full model includes a motion-processing subsystem, which is fully integrated with the occlusion system. Although the motion aspects of tie system will not be discussed in detail, it is important to note that such a multimodal model represents a first step toward simulating the interactions between illusory contours generated in different domains. Of particular interest in this model, motion-induced contour information feeds back into the luminance-defined channels, allowing for emulation of second-order contour induction, in which motion-defined inducers generate illusory contours (Kellman \& Loukides, 1987; Prazdny, 1986).

After generating oriented and end-stopped cell responses via simple receptive field models, Finkel and Edelman (1989) pooled end-stopped cell activity over similar orientations at the same location into a "wide- a

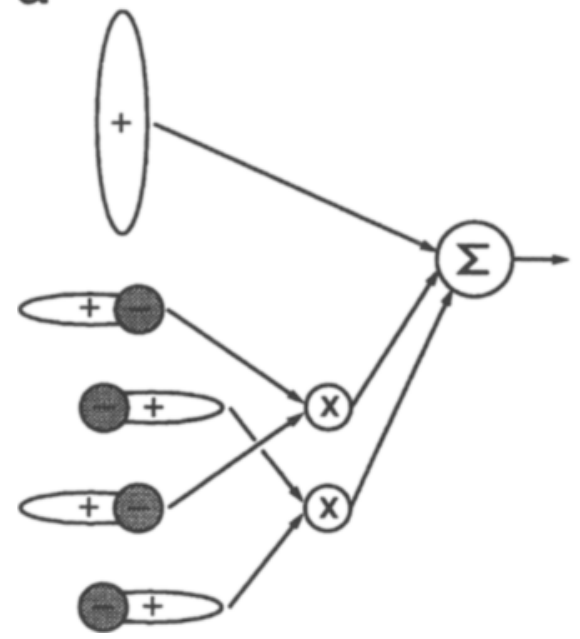

b

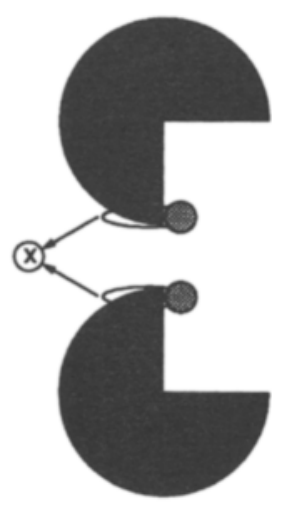

Figure 21. (a) In the model of Peterhans, von der Heydt, and Baumgartner (1986), signals for real and illusory contours additively converge at the same neuron. (b) End-stopped cells respond to the corners in a Kanizsa figure leading to illusory contour formation. From "Mechanisms of Contour Perception in Monkey Visual Cortex," by E. Peterhans and R. von der Heydt, 1989, Journal of Neuroscience, 9, pp. 1749-1763 (Figures 15-16). Copyright 1989 by the Society for Neuroscience. Adapted with permission. 


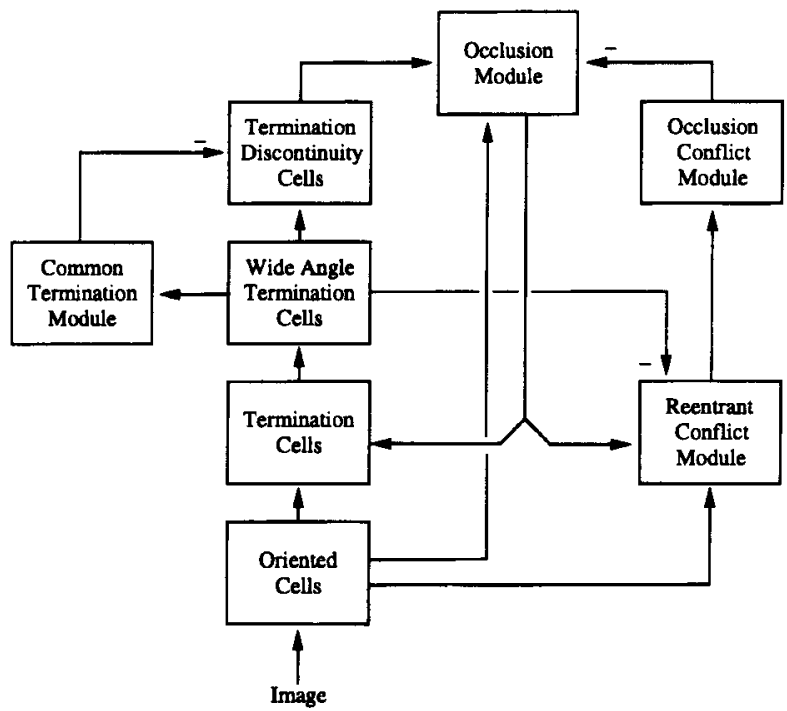

Figure 22. The luminance-based processing modules of the model of Finkel and Edelman (1989). From "Integration of Distributed Cortical Systems by Reentry: A Computer Simulation of Interactive Functionally Segregated Visual Areas," by L. H. Finkel and G. M. Edelman, 1989, Journal of Neuroscience, 9, pp. 3188-3208 (Figure 1). Copyright 1989 by the Society for Neuroscience. Adapted with permission.

angle" representation. They then specified that termination discontinuities - ecological cues to occlusion-could arise only if at least three such wide-angle cell pools were active along a line perpendicular to the center (mean) orientation of these wide-angle pools. Finally, perpendicular contour completion between termination discontinuities occurred at the occlusion module. Finkel and Edelman speculated that the contour representation at the occlusion module fed back to early levels via a "reentrant" system. Reentrant signals would allow the system to mediate conflicts between real and illusory contours, sharpening or eliminating contours as appropriate. Conflict resolution would be required, for example, when a real line crossed an illusory contour. Besides addressing this form of figural interference, Finkel and Edelman's model also included a mechanism that may account for some small-scale interference effects. At the termination discontinuity level, activity was inhibited if the common termination module determined that some of the end-stopped activity was actually arising from a natural corner or vertex-features known to inhibit contour formation - rather than a line termination.

The importance of line terminations as occlusion indicators for scene segmentation is the basic tenet of Finkel and Edelman's (1989) model. The structure of the resulting model mandates that illusory contour formation is mediated only by these terminations. Thus illusory contours formed by edge inducers must result from end-stopped activity arising from termination cell activity at the corners of inducing elements, as Figure $21 \mathrm{~b}$ indicates for the simpler model of Peterhans et al. (1986). There is neither parallel completion nor mediation of contour strength by parallel (edge) information within this model.
Finkel and Sajda (1992). Finkel and Sajda (1992, 1994; Sajda \& Finkel, 1992a, 1992b, 1995) have presented an intermediate level visual model for segmentation of scenic elements, including illusory figures, into different depth planes. The primary mechanism for illusory contour formation is based on perpendicular completion between line ends and corners, much as the earlier model of Finkel and Edelman (1989). As such, one would expect that the model of Finkel and Sajda should behave in a manner qualitatively similar to that of Finkel and Edelman when confronted with variations in low-level inducer determinants. However, Finkel and Sajda's model is geared toward representation of sophisticated surface perceptions, rather than the effect of low-level factors, and therefore may be able to emulate certain high-level determinants, especially those related to the effects of perceived depth on illusory contour formation.

Nakayama et al. (1990) have pointed out that local edge information can be used to determine whether boundaries are "intrinsic" to a particular object or whether they are "extrinsic" to the object--signifying occlusion by another object. In Finkel and Sajda's (1992) model, local and global cues to boundary "ownership" are linked through a series of completion mechanisms, resulting not only in a determination of which contours belong to the same surface, but also in full representations of all surface contours, both modal and amodal, stratified into distinct depth planes. Cues to contour ownership employed by Finkel and Sajda include closure, similarity and proximity, local concavities, and direction of line terminations. By combining these cues through a series of local interactions the model arrives at a stratified representation of surfaces, which may be refined via feedback of real and hypothesized (either illusory or amodal) contours to earlier stages.

Kellman and Shipley (1991). Kellman and Shipley have abstracted the line-end theory of contour induction a step further, claiming that first-order spatial and spatiotemporal discontinuities are the generating features of illusory contours. These discontinuities can lead to illusory contour formation if they are relatable - that is, if they satisfy certain constraints concerning the intersection of the extensions of lines perpendicular to the inducing line or parallel to the edge associated with inducer corners, as is indicated by Figure 11. Unlike in the earlier line-end models, Kellman and Shipley propose that contour completion can proceed in directions parallel to the supporting edges of the inducers, provided that these edges are associated with discontinuities. Because contours are initially induced only by discontinuities, this model is not technically considered a hybrid system, although its use of both parallel completion and mediation of contour strength by edge information positions the model at the border between line-end and hybrid systems.

The degree to which discontinuities are relatable determines the contour strength, a hypothesis supported by several recent experiments (Shipley \& Kellman, 1992b). As in the Finkel and Edelman model, edge-induced illusory contours are induced by the discontinuities at the corners of the inducing elements (Figure 21b). Shipley and Kell- 
man (1992a) presented data indicating that contour clarity is a linear function of the support ratio over a range of 0.3 to 0.8 , where the length of the supported region and the length of the unsupported region were varied independently. Banton and Levi (1992) have reported a monotonic, but nonlinear, relation. By scaling the size of their stimuli, with inducers and gaps increasing proportionately, Shipley and Kellman showed that clarity is a fixed function of support ratio over a range of absolute stimulus sizes. Their theory holds that illusory contour strength is directly proportional to the support ratio, regardless of the absolute degree of support, the proximity of the inducers, or the size of the inducers. Kellman and Shipley (1991; Shipley \& Kellman, 1992a) noted that the support ratio must be incorporated into the mechanics of the illusory contour interpolation process, but they provided no further computational details. Note that this model is not strictly neural-Shipley and Kellman presented no details of how their system should be implemented-but could be linked to a neural architecture with modest efforts.

\section{The Hybrid Models}

Grossberg and Mingolla (1985a, 1985b). Grossberg and Mingolla (1985a, 1985b, 1987a, 1987b) proposed the existence of two parallel systems in the visual stream, a boundary contour system (BCS) for establishing the boundaries of objects, and a feature contour system (FCS) for establishing the color and brightness of these objects. Illusory contour formation is performed by the BCS through interactions between spatial arrays of cells which represent orientations of boundaries at every position. It should be noted that in Grossberg and Mingolla's approach, the visibility of illusory contours depends on the FCS's establishing local brightness differences between the regions on either side of the contour. As Figure 23 indicates, the first stage of the BCS consists of an array of oriented contrast detectors at each spatial location. The inputs to these detectors consist not of the raw image data, but rather of the output of a competitive on-center, off-surround preprocessing stage designed to emulate the partial brightness normalization performed within the retina. In the second BCS stage, first-stage cells with the same orientational preference but opposite contrast preference are pooled to yield a contrast-independent boundary representation.

Because units sensitive to oriented image contrasts may be activated by stimuli falling anywhere within their extended receptive fields, a degree of positional uncertainty is inevitably present in the distribution of responses of fields of such units. This is particularly true at line ends and corners, where the lack of definite orientation information leads to severe ambiguity. Grossberg and Mingolla posited the existence of a two-stage competitive mechanism designed to overcome these uncertainties of local measurement. These processes establish "end cuts" at the end of each line--activity at cells with orientation preferences perpendicular to the line itself. In the first competitive stage, cells with the same orientational preference in neighboring locations compete, functionally generating end stopping in the sense that survivors of the competition will tend to respond most strongly to the ends of lines or corners. In the second competitive stage, cells at the same location, but with different orientational preferences, compete. The first step in end-cut generation occurs in the first competitive stage as strong cell activations along the edges of a line inhibit the weaker activations near the line end. In the second competitive stage, these weakened activations disinhibit perpendicular cell activations near the end of the line, generating the perpendicular end cut.

The actual illusory contours in the BCS are generated via a process of oriented long-range completion. In contrast with the previously discussed researchers, Grossberg and Mingolla believe that completion always occurs in directions approximately parallel (collinear) to the orientation preferred by the inducing units of the second competitive stage. Edges directly activate these inducing units, whereas line ends rely on the first and second competitive stages to indirectly activate inducing units through end cutting. To accomplish contour completion, bipole filters with bowtie-shaped receptive fields combine inputs from spatially disparate cells whose receptive field centers fall roughly along a common line or curve, and whose orientational preferences are consistent with that line or curve. In discussing their recent psychophysical experiments, Field, Hayes, and Hess (1993) have recently referred to such a mechanism as an "association field." The relative contribution of a cell to a bipole filter depends on that cell's position, alignment, and orientation with respect to the bipole. A bipole cell fires only if both sides of its bipartite receptive field are sufficiently excited. These cells feed back to earlier stages, providing orientational information to locations where there are no bottom-up signals supported by image contrast. By tuning the extent to which oriented cells in various positions, alignments, and orientations (with respect to the bipole) contribute to bipole activity, the discrete (on or off) long-range completion scheme employed by Peterhans et al. (1986) and Finkel and Edelman (1989) can be accomplished with a bipole filter, as can a measure similar to the relatability of Kellman and Shipley (1991).

Once boundaries have been determined by the BCS, the filling-in process of the FCS establishes brightness levels throughout the image. Within the FCS, retinal brightness signals spread out diffusively until blocked by BCS boundary signals, restoring brightness information removed during BCS processing. Illusory contours are perceived only when the FCS indicates that BCS contours represent boundaries between regions of two locally different brightnesses. Note that in cases of stimuli with opposite-contrast inducing elements, local brightness effects will not necessarily result in a global brightness percept.

Grossberg (1994). Drawing upon a large body of earlier work (Grossberg, 1987a, 1987b; Grossberg \& Marshall, 1989; Grossberg \& Mingolla, 1985a, 1985b, 1987a, 1987b; Grossberg \& Rudd, 1989, 1992), Grossberg (1994) has recently synthesized an advanced theory of human vision model called FACADE (from form and color and depth). As the name indicates, this BCS/FCS-based theory incorporates not only form, the one aspect that has been dis- 


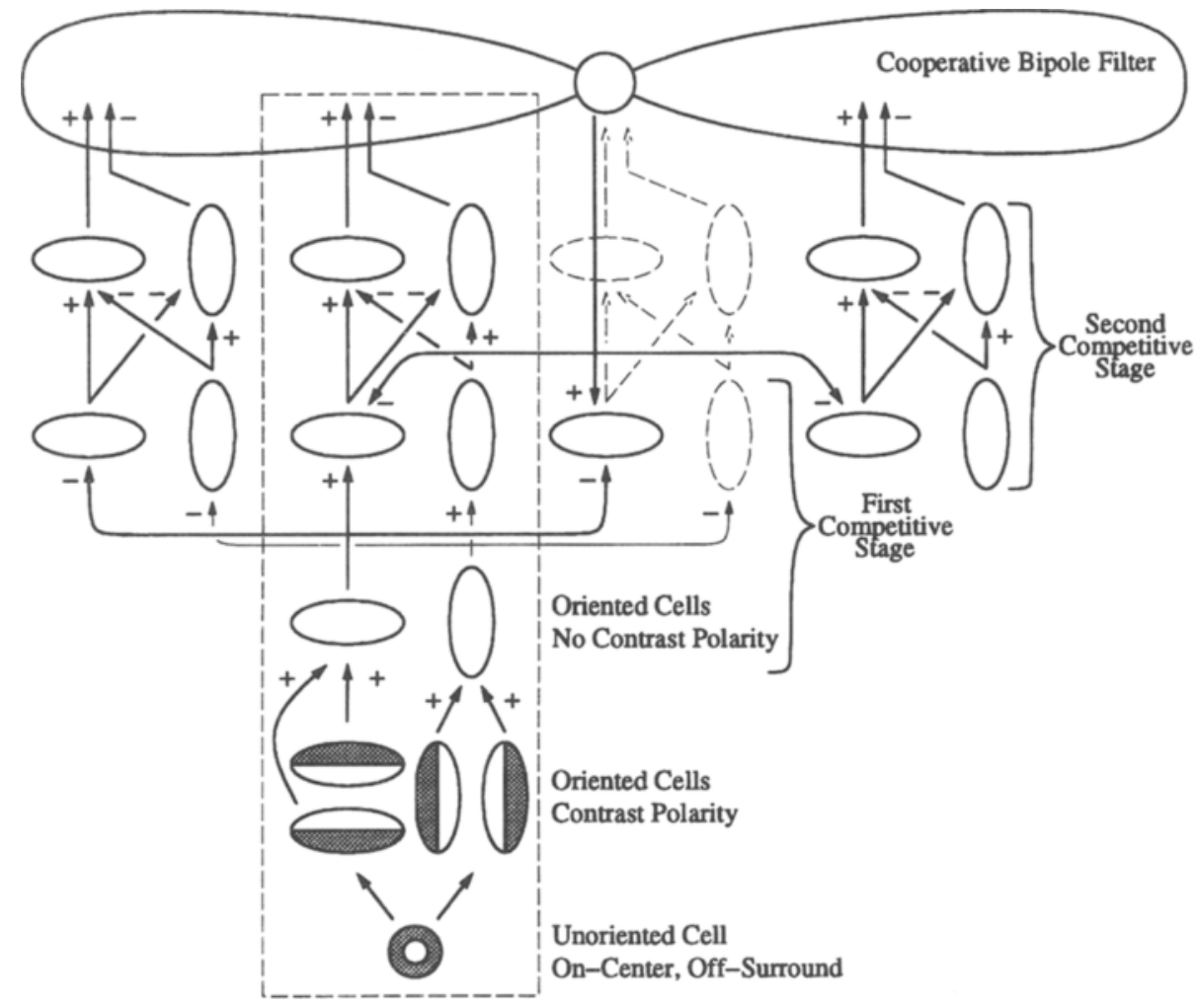

Figure 23. The boundary contour system of Grossberg and Mingolla (1985a, 1985b, 1987a, 1987b). From "Neural Dynamies of Perceptual Grouping: Textures, Boundaries, and Emergent Segmentations," by S. Grossberg and E. Mingolla, 1985, Perception \& Psychophysics, 38, pp. 141-171 (Figure 30). Copyright 1985 by the Psychonomic Society, Inc. Adapted with permission.

cussed above, but also color and stereo depth (and also, though not indicated by the name, motion). Of particular interest to this review are the advances that Grossberg has made in the area of how depth interacts with contour formation. The basic premise behind depth perception in the FACADE system is that local depth information from binocular and monocular cues forces individual boundaries onto independent BCS stratification planes. Inhibition from large to small scales effects resolution of ambiguous depths and eliminates boundaries from appearing in incorrect stratification planes. Once boundaries have been stratified, modal (illusory) and amodal contour completion are subserved by the same mechanisms acting on different planes. After all boundaries have been established, boundary signals from large scales are reintegrated with smaller scales for the purpose of brightness and color determination. This process ensures that subsequent FCS filling in leads to veridical perception, preventing the assignment of individual brightnesses and colors to multiple objects at the same position, but on different depth planesa mishap that would make all occluding objects appear transparent. Reciprocal interactions between processing streams ensure that BCS boundaries and FCS surfaces are mutually consistent. Both modal and amodal contours are established within the FACADE system, but only modal contours are visible.
Heitger and von der Heydt (1993). Heitger and von der Heydt (1993) have recently presented a model, loosely based on the earlier line-end model of Peterhans et al. (1986), which employs the sophisticated edge and discontinuity detectors described in Heitger et al. (1992). Rather than the simple linear grouping of the line-end models, however, signals from these detectors are combined via a two-lobed grouping field which closely resembles Grossberg and Mingolla's bipole filter. As for the bipole filter, the weights of the grouping field can be assigned so that they mirror psychophysical data regarding several low-level determinants. A multiplicative gating of lobe pairs ensures that completion is always in an inward direction. By allowing "bent" combinations of grouping field lobes, the system can produce curved illusory contours. After grouping, and a competitive stage that eliminates contradictory orientations at the same position, fuzzy bands of activity indicate the presence and strength of both illusory and real contours. In a collection of impressive simulations, the maximal ridges along these bands closely approximate human illusory contour perception.

Unlike the line-end models discussed above, the model of Heitger and von der Heydt (1993) allows grouping in both parallel and perpendicular directions. Inputs to the grouping field are not simple line-end activities, but a complex combination of ortho (line-end) and para (edge or line) 
activity. The degree to which ortho and para activity contribute to grouping is dynamically determined by a weighting factor $\kappa$, which indicates the "cornerness" of the stimuli at each point in space. The larger $\kappa$, the higher the relative contribution of the para signals. This model makes explicit distinction between parallel and perpendicular completion, with different degrees of completion being employed as determined by the computation of $\kappa$. This approach is fundamentally different from that taken in the BCS, in which parallel and perpendicular completion result from the same mechanism operating in different spatial environments-an implicit distinction between the two forms of completion. Noting that line ends do not specify the exact orientation of the occluding object, but merely suggest the most probable orientation, Heitger and von der Heydt have included an orientational tolerance for ortho completion but not for para completion, since the latter is tightly constrained by inducer edge orientations.

A potentially powerful aspect of Heitger and von der Heydt's (1993) model is the manner in which figureground assignments are made. By maintaining tags indicating the direction of termination of edges and corners and allowing completions only between certain combinations of termination directions, the output of the system indicates the likely depth relations between adjacent objects. Not only does this mechanism facilitate the interpretation of illusory figures, it makes the system a potentially powerful image-understanding tool for real-world imagery, as is evidenced by several examples presented by the authors. Note, however, that precluding completion across certain termination directions may destroy the ability of the network to model illusory contours with inducers on both the inside and the outside of the illusory figure (as in Figure $4 \mathrm{~b}$ ).

\section{DEVELOPING AND EVALUATING NEURAL MODELS}

Neural systems have evolved to a sophistication commensurate for modeling advanced illusory contour phenomena only within the past few years. Widespread implementation of the models has lagged behind their initial theoretical formulation, but impressive simulations have recently surfaced. In particular, recent BCS/FCS simulations and interactions have been employed to reproduce results on the effect of line spacing and support ratio on contour strength (Lesher, 1993), illusory brightness effects in line-end figures (Gove, Grossberg, \& Mingolla, 1994a, 1994b), and visual persistence of illusory contours (Francis, Grossberg, \& Mingolla, 1994). Using their hybrid model, Heitger and von der Heydt (1993) have demonstrated simulations of curved illusory contours and contours generated by inducers on the inside of the illusory figure. Sajda \& Finkel (1992a, 1992b, 1995; Finkel \& Sajda, 1994) have demonstrated depth ordering of illusory figures and inducers. Yet despite these advances, current models only explicitly support a limited set of the low-level determinants discussed in this paper, with almost no support for the high-level determinants.
For each low- and high-level determinant, I will outline and justify the constraints that the psychophysical data place on neural models of illusory contour formation. An analysis of the degree to which current models fulfill these constraints will also be provided. Because no modelers have attempted to reconcile their neural models with the entire body of psychophysical data, some models support experimental data overlooked by their originators. More often, however, the models fall short of explaining previously unconsidered phenomena, and in some cases, are the subjects of unsupported explanatory claims. Before dealing with the modeling constraints of specific determinants, I will discuss some general issues regarding the inherent differences between line-end and edge inducers and how these differences influence modeling of illusory contour formation.

\section{Edge and Line-End Inducers}

The supposition of line-end and discontinuity models is that all contour completion is in a direction roughly perpendicular to the inducing element or to that specific part of the stimulus doing the inducing (e.g., a corner). This assumption can be discounted on two grounds. First, numerous researchers have shown that the extent of edge support (or more recently, the support ratio) has a significant effect on illusory contour strength and illusory brightness. The models of Peterhans et al. (1986) and Finkel and Edelman (1989) determine contour strength solely by relation of perpendicular elements. Conversely, contour strength in the model of Kellman and Shipley (1991) is determined by inducer relatability, a concept that applies to both line-end and edge inducers, and support ratio, which applies only to edge inducers. Thus Kellman and Shipley's model, but none of the stricter line-end models, survives this first finding. The documented effects of the degree of edge support precludes any models that do not at least include mediation of contour strength by something akin to support ratio.

The second argument for discounting the basic premise of line-end models is that a number of researchers have demonstrated illusory-contour-inducing stimuli containing no line ends, corners, or other such discontinuities (Grossberg \& Mingolla, 1985a; Lesher \& Mingolla, 1993; Shapley \& Gordon, 1987). The existence of contours in the absence of discontinuities precludes models that rely on such features for contour induction, such as that of Kellman and Shipley (1991), although the use of multiple spatial scales might allow these systems to sidestep this apparent discrepancy, as described earlier. Without significant modifications, none of the strict line-end models can be reconciled with basic psychophysical data. The model of Heitger and von der Heydt (1993; which for the sake of brevity will henceforth be referred to as the CMNCPcomputational model of neural contour processing-as taken from the title of their paper) suggests the form that the necessary modifications might take. The completion mechanisms of Kellman and Shipley's model might be consistent with rudimentary illusory contour data, but development of neural mechanisms and of methods of scale interactions are required before further evaluation is possible. 
Both of the hybrid models discussed in this article, the BCS/FCS and the CMNCP, contain mechanisms that provide them with the potential to model illusory contour formation with both edge and line-end inducers. However, the CMNCP perhaps has an advantage over the standard $\mathrm{BCS} / \mathrm{FCS}$ in modeling line-end contour formation. Lesher and Mingolla (1993) have shown that line-end inducers in a Varin configuration lead to significantly higher illusory contour strengths than do solid edge inducers. Reconciling the fact that a few highly localized inducers can yield stronger contours than can an extended edge is difficult in a model such as the BCS, which contains a single pathway for completion and a filter that spatially integrates over regions of completion. One must suppose that the end-cut signals generated by competitive mechanisms are significantly stronger than the signals corresponding to extended edges. In the CMNCP, where the relative contributions of perpendicular and parallel completion can easily be biased, there is no corresponding problem. Recent advances in the BCS/FCS system, however, may rectify this apparent shortcoming. Gove and colleagues (Gove, 1993; Gove et al., 1994a, 1994b) have proposed a mechanism to accomplish dissimilation -brightening at the ends of lineswhich also has the effect of strengthening the functional end stopping performed by the competitive stages of the BCS. When combined with the surround inhibition of the first competitive stage, which weakens edge responses more than end-cut responses, BCS end-cut activities can easily surpass edge activities in magnitude. A more radical approach has been taken by Manjunath and Chellappa (1993), who have added a separate pool of end-stopped cells to a BCS-like system for texture discrimination.

\section{Brightness Effects}

The mention of mechanisms for modeling dissimilation raises the most significant shortcoming in the field of the modeling of illusory forms - namely, the failure of any model except the BCS/FCS to address the issue of illusory brightness. Even with edge inducers - let alone line endsDresp (1992) has shown that simultaneous brightness contrast is insufficient to explain the homogeneous appearance of illusory figures. There must be additional mechanisms to fill in these forms, as well as a mechanism that can produce dissimilation effects. The feature contour system of the BCS/FCS allows local brightness contrast and dissimilation mechanisms to determine the global appearance of illusory figures via a diffusive process with boundaries to the diffusion determined by the BCS. No quantitative studies involving the effects of low-level stimuli variations on FCS brightness levels have yet been undertaken, so it is unclear how well this system will perform. However, given its ability to reflect a number of simultaneous brightness contrast effects (Grossberg \& Todorović, 1988), one can predict that it will make accurate brightness predictions under parametric stimulus variations for which there is a high correlation between brightness contrast and illusory brightness, such as inducer proximity and size (Dresp, 1992; Dresp et al., 1990).

\section{Low-Level Determinants}

Before discussion of individual low-level determinants, it will be useful to introduce a construct shared by the more sophisticated models of illusory contour formation. The bipole filter of the BCS, the grouping field of the CMNCP, and the association field of Field et al. (1992) all share a common purpose-determining when contour completion should occur and establishing the resulting illusory contour strength. These filters, which I will refer to by the generic name of completion filters, are similar from a mechanistic standpoint as well: they integrate edge and line-end information over two separate receptive fields (lobes) and fire only when both fields receive sufficient excitation. The psychophysical constraint that illusory contours appear only between two inducing regions and do not extend outward from a single inducer mandates such a dual-lobe arrangement. The known effects of inducer separation, support ratio, and degree of alignment mandate that the relative contribution of each cell to a completion filter depends on the relative position and orientation between the cell and the completion filter. Specific constraints placed on the completion filter's receptive field profile will be discussed below in the relevant sections.

The relation between any pair of cells, or between a cell and a completion filter, can be uniquely characterized by three parameters, as is indicated by the schematic of Figure 24 (Grossberg \& Mingolla, 1985a, 1985b). These parameters are the Euclidean distance $r$ between the center of the cells' receptive fields, the angle $\theta$ of one cell with respect to the other cell's orientational preference, and the angle $\phi$ between the orientational preferences of the two cells. Any receptive field can be characterized as some function $f(r, \theta, \phi)$ of these three parameters. Simple receptive fields, such as those of like-oriented cells competing across space (as in the first competitive stage of the BCS), can be characterized by a separable function:

$$
f(r, \theta, \phi)=f_{r}(r) f_{\theta}(\theta) f_{\phi}(\phi),
$$

with some degenerate or trivial $f_{x}$-for this case, something along the lines of

$$
\begin{aligned}
& f_{r}(r)=\text { difference of Gaussians, } \\
& f_{\theta}(\theta)=1, \\
& f_{\phi}(\phi)=\delta(\phi),
\end{aligned}
$$

where $\theta$ has no effect on the receptive field. Starting from the constraints of the simplest of low-level determinants, I will construct the receptive field profile of the completion filter and show that it must depend nontrivially on all three relational parameters.

Spatial extent and proximity of edge inducers. For edge-inducing elements, illusory contour strength appears to be closely related to the support ratio. With all other stimulus aspects kept constant, this implies that the strength of completion must increase as inducing elements move closer together and as their region of support grows larger. These constraints are satisfied by completion mechanisms that employ weighted spatial integration along the com- 


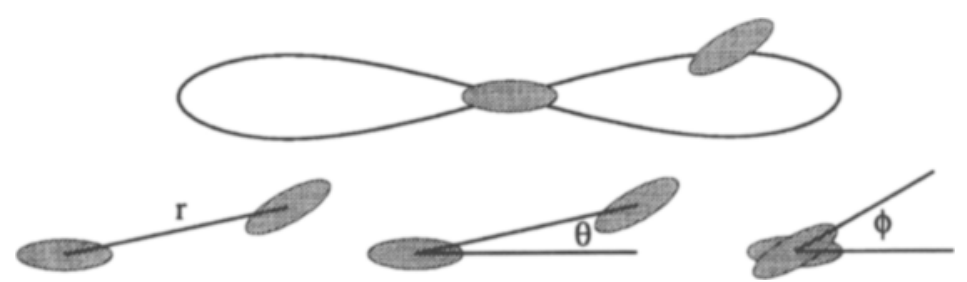

Figure 24. The relation between any two cells in a neural model can be uniquely characterized by three parameters. A completion filter with weights $f(r, \theta, \phi)$ depending upon these parameters can be defined so that it closely models the effects of inducer alignment, proximity, and support on illusory contour strength.

pletion path, with weights decreasing monotonically from the completion centroid outward. That is, a completion filter with a decreasing $f(r)$ can reproduce these effects. Decreasing the distance between inducers will cause the inducers to fall in more highly weighted regions, thereby increasing completion strength. Increasing inducer support will cause the additional integration of previously unintegrated regions, again increasing completion strength. The grouping field of the CMNCP and the bipole filter of the BCS both use weighted integrations of this form, whereas other models of illusory contour formation do not include such mechanisms. Note that both of these models contain competitive, nonlinear processes prior to completion, which may significantly alter the strengths and spatial distributions of the signals that act as inputs to the completion mechanisms, with the BCS containing a feedback loop that can further transform the signals as described below. Thus, even if the grouping weights fall off as described above, mathematical analysis (or, if intractable, extensive simulation) of the entire system is required in order to establish the appropriate support and proximity trends. Lesher and colleagues (Lesher, 1993; Lesher, Grossberg, \& Mingolla, 1994) have recently verified these trends in the BCS through a series of one-dimensional simulations.

Reconciling completion strength with the support ratio findings of Shipley and Kellman (1992b) and Banton and Levi (1992) requires more than just modeling the effects of inducer proximity and amount of absolute support. The fall-off in grouping weights must reflect the psychophysically observed trends for the ratio of supported length to the length of the entire contour (the sum of distance between inducers and supported length). With an infinite number of distinct distance/support pairs leading to the same support ratio, and thus the same completion strength, it is a relatively simple process to show that no single set (scale) of discrete completion filter weights can fulfill the support ratio constraint, although there may be ways to combine completion filters of multiple scales in order to remain consistent with this constraint. On the basis of the conflicting data between edge and line-end inducers, however, it is not yet clear that support ratio represents an invariant determinant of illusory contour strength, even for edge inducers. Additional research into the relationship between support ratio and inducer type is necessary before explicit modeling problems can be fully addressed.
Proximity and number of line-end inducers. Varying the spacing, number, and support ratio of line-end inducers affects illusory contour strength quite differently than the equivalent manipulations of edge inducers. When a constant spacing is maintained between line-end inducers, illusory contour strength increases monotonically as the number of line ends increases. The weighted integration of the completion filter remains consistent with this psychophysical data: additional lines can only increase the completion filter response. However, as the number of lines increases within a fixed region (necessarily leading to a decreased spacing between lines), an initial increase in contour strength is psychophysically observed, followed by a strength decrease. This finding cannot be reconciled with just the basic completion filter; an increased number of lines should always result in increased contour strength, as should decreased distance between neighboring lines.

The inverted-U observed with line proximity can be explained by introducing a distance-dependent interference (competition) between parallel lines or line ends, or, more accurately, between detectors signaling these features (Lesher \& Mingolla, 1993). Note that the CMNCP, as well as all strict line-end models, would require such competitive interactions only between end-stopped cells, since the edge detectors do not participate in perpendicular completion. Conversely, the BCS would require such interactions only between edge-sensitive cells since it contains no distinct pool of end-stopped cells, but functionally generates end stopping via competition between these edgesensitive cells. This dichotomy indicates a possible advantage for the former model, since it is the illusory contour rather than the lines themselves that are degraded when the inducing lines are close together.

Fortunately, one need not posit novel mechanisms to explain interference effects between proximal lines or line ends, but rather may exploit existing mechanisms that subserve other purposes. Many models of end-stopped cells employ some form of implicit or explicit lateral inhibition to achieve their end-stopping capabilities (Dobbins, Zucker, \& Cynader, 1989; Heitger et al., 1992). Similarly, the first competitive stage of the BCS consists of spatial competitions between like-oriented cells. Note, however, that the deleterious effect of neighboring parallel lines on illusory contour strength has been observed at spacings many times the width of the lines themselves - beyond the distance at 
which conventional end-stopping models would include lateral inhibitions and beyond the spatial extents employed in most previous BCS implementations (but see Grossberg, Mingolla, \& Williamson, 1994; Pessoa, Mingolla, \& Neumann, 1994). With line interactions in place, employing the completion filter to model the inverted-U effect of line proximity (or number of lines if their extent is kept constant) becomes a matter of balancing the tendency of completion strength to increase as lines move into regions of higher filter weighting with the tendency of the strength of each line's signal to decrease as neighboring lines inhibit one another. The inverted- $U$ has recently been qualitatively modeled within a one-dimensional BCS implementation (Lesher, 1993; Lesher et al., 1994).

Inducer luminance and contrast. It appears that illusory contour strength decreases with decreasing inducer contrast. Assuming a correlation between inducer contrast and strength of edge and line-end signals, both the BCS and CMNCP should be able to reproduce these results. Since various inducers need not be of the same contrast, contour completion should be polarity independent, as it is in both models. The lack of illusory contours under conditions of inducer/background isoluminance indicates that contour formation may occur solely in luminance channels, although there is some evidence that inducer/background color choice can affect luminance difference detection thresholds for illusory contours (de Weert, 1983). Grossberg (1987a, 1987b) has extended the description of the original BCS/FCS to employ separate sets of double-opponent (red-green, blue-yellow, white-black) channels. By allowing completion only between boundary signals generated by luminance differences (as opposed to color differences), the BCS approximates the data on isoluminant contour formation.

Inducer alignment. Psychophysical data on the alignment of edge inducers mandate that completion between adjacent inducers be curvilinear, with decreasing strength as the degree of curvature increases, although under certain conditions the contour may contain a discontinuity or corner. In addition, contour strength decreases as increasing degrees of curve inflection are required for smooth completion (the completion of Figure $11 \mathrm{~b}$ would require an inflection). In the completion filter paradigm, the completion strength or relatability between two edges or line ends can be veridically modeled by forcing the filter's receptive field to depend nontrivially on all three relational parameters $-r, \theta$, and $\phi$.

By definition, a completion filter should fire if it lies tangent to an illusory (or real) contour. The strongest illusory contour strength should result when the inducing edges lie along a common line, as depicted in Figure 25a. For completion filters tangent to this line, $\theta=\phi=0$. For completion filters of the same orientation, but in different positions, as in Figure 25b, $\theta$ becomes nonzero while $\phi$ remains zero. Psychophysically, we observe that there is no illusory contour apparent at the completion filter's position: The response of this filter must be less than that of the tangential filter of Figure 25a. Thus, for this value of $\phi=0$, the receptive field profile must fall off with increasing $|\theta|$. For completion filters at the same position, but of different orientations, as in Figure 25c, both $\phi$ and $\theta$ become nonzero. The effect of varying $\phi$ can be isolated by altering the inducer configuration as in Figure $25 \mathrm{~d}$, in which $\theta=0$. There is no contour apparent at this nontangential orientation, so the completion filter response must drop off with increasing magnitude of $\phi$, as it did above for $\theta$.

The simple constraints outlined above for independent variations in $\phi$ and $\theta$ have been defined with reference to the optimal completion filter, with $\phi=\theta=0$. However, $\phi=$ $\theta=0$ only describes the optimal filter for the special case in which the inducer edges lie along a common line. Figures $25 \mathrm{e}$ and $25 \mathrm{f}$ depict optimal completion filters for more complex inducing stimuli, under straight-line and parabolic completion paradigms, respectively. In Figure $25 \mathrm{e}$, the optimal filter is defined as having $\phi=\theta$, such that the linear extensions of the inducing edges intersect at the centroid of the completion filter. In Figure 25f, the optimal filter is tangent to the parabola defined by the two inducing edges. In the parabolic case, $\phi$ must be greater than $\theta$ in the optimal completion filter. Of course, one could just as easily define optimality in terms of circular contours (as Ullman did), or any other curvilinear construct. In any event, the response of the completion filter must fall off as $\theta$ and $\phi$ vary from their optimal combination, as indicated by Figure $25 \mathrm{~g}$ for parabolic completion.

As yet, we have only considered how the response of a completion filter changes relative to the optimal relative parameter set $(\theta, \phi)$ for a given inducing stimulus. How do we relate our psychophysical knowledge about the effects of degree of alignment on absolute contour strength as the inducing stimulus is altered? Contour strength decreases with decreasing alignment, or alternatively, with increasing degrees of curvature, as is indicated by Figures $25 f$ and $25 \mathrm{~h}$. This effect is emulated by the completion filter if its response is greatest for $\theta=0$ (straight line completion) and falls off monotonically as the magnitude of $\theta$ increases.

Given all of the completion filter constraints discussed above, the strength of the filter response must decrease with increasing $r$, increasing $|\theta|$, and increasing distance from the optimal $(\phi, \theta)$ pair as defined by the particular curve scheme being employed (e.g., in a linear scheme, $\phi=\theta$ would be optimal and contour strength would decrease with increasing $|\phi-\theta|)$. The sophisticated completion filter of the $\mathrm{BCS}$ uses a separable response profile:

$$
f(r, \theta, \phi)=f_{r}(r) f_{\theta}(\theta) f_{\psi}(\theta-\phi),
$$

as does the simpler filter of the CMNCP. Although a separable filter is easier to define and implement, it has the serious drawback of eliminating all curvature schemes other than the piecewise linear completion of Figure 25e. More sophisticated methods require joint knowledge of $\phi$ and $\theta$ (and, for some schemes, even $r$ ).

The bipole filter of Grossberg and Mingolla (1985a, 1985b) encompasses all the constraints provided above, with the maximal nonaligned contributions occurring at $\phi=\theta$. As indicated by the recent data of Field et al. (1992) and the propensity for curved contours rather than those with discontinuities, this configuration is probably suboptimal because it tends to lead to piecewise linear con- 

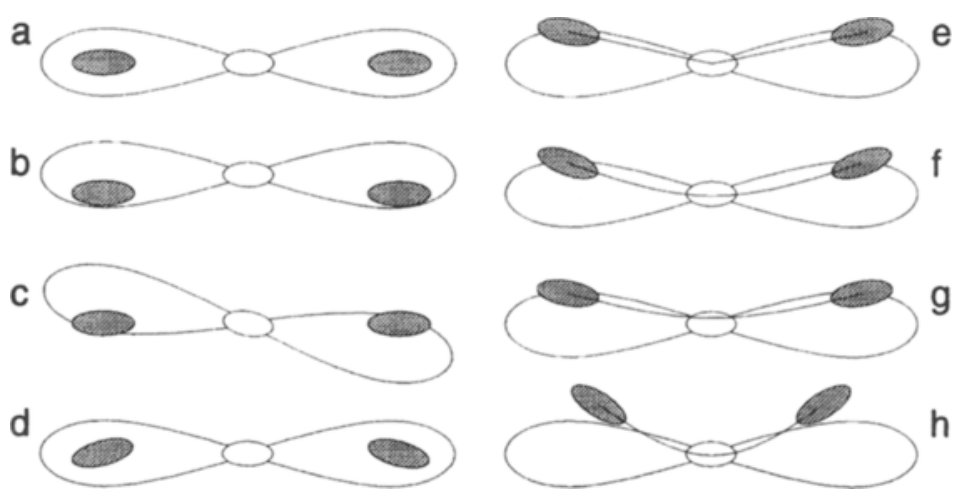

Figure 25. The effect of various alignments on completion filter response: (a) optimal tangential completion filter with $\theta=\phi=0$, (b) suboptimal filter with $\theta \neq 0, \phi=0$, (c) suboptimal filter with $\phi \neq 0, \theta=0$, (d) suboptimal filter with $\phi \neq 0$, $\theta=0$, (e) optimal filter for piecewise linear completion, $\phi=\theta$, (f) optimal filter for parabolic completion, $\phi>\theta$, (g) suboptimal filter for parabolic completion, $\phi=\theta$, (h) optimal filter for parabolic completion, but weaker response than for (f).

tours intersecting at bipole centroids-although the joint action of many bipole filters and the competitive interactions within the feedback loop may restore true curvature. As described above, a more propitious choice for the occurrence of maximal contribution would lead to contours in the form of parabolas or circles, but at the cost of losing filter separability. The grouping field employed by Heitger and von der Heydt (1993) is simpler than the bipole filter, allowing cells to contribute only if they have the same orientational preference as the grouping filter; that is, weights are set to zero when $\phi \neq 0$. By itself, allowing only two nontrivial degrees of filter freedom $(r$ and $\theta$ ) would have the effect of precluding curved illusory contours, but in a subsequent step filter lobes from a specific set of orientations are combined in a weighted fashion. This process brings the third degree of freedom $(\phi)$ back into the completion mechanism, but only to a limited extent: the orientation combinations are coarse, with most $\phi$ information lost during the grouping filter process. Although computationally more efficient than the bipole filter, this system lacks the flexibility and modeling power of the bipole filter, gained through the finer dependence on $\theta$ and $\phi$ in determining completion strength. Like the bipole filter, piecewise linear groupings are preferred to true curved completion, although the conglomerate action of many grouping fields does lead to curved completion. Both the BCS and the CMNCP would benefit from completion filters explicitly tuned to produce curved contours. In the BCS, implementation of such filters would merely involve replacement of the standard filter weights, whereas in the CMNCP, the system would require modifications to handle completion filters with 3 nontrivial degrees of free$\operatorname{dom}(r, \theta$, and $\phi)$ rather than the 2 degrees of freedom currently supported. Gove and colleagues (Gove, 1993; Gove et al., 1994a) have recently demonstrated BCS bipole filters with circular completion preferences.

One of the most powerful features of the BCS is its feedback loop, which theoretically allows for the capture of distinct, curved illusory contours which a solely feed-forward system like the CMNCP can accomplish only through (currently) artificial postprocessing methods. The sharpening and selection of illusory contours in the CMNCP involves the identification and extraction of "ridges" of high activity-a process yet to be neurally defined by Heitger and von der Heydt (1993). Besides sharpening contours, the feedback loop of the BCS also serves to eliminate spurious and overlapping contours that would be maintained by the CMNCP and other models. While CMNCP simulation results indicate that multiple illusory percepts are visible at the same time (e.g., both the Kanizsa square and the circular inducers are completed), the competitive feedback of the BCS renders such multiple percepts unstable and, in agreement with psychophysical data, ensures that at any given time, only a single percept will be visible. The primary drawback of the feedback loop (and of the BCS/ FCS in general), from the perspective of actually simulating illusory contour formation, is that it is computationally expensive. This fact has allowed the CMNCP to hold the advantage of actually demonstrating a number of tangible illusory contour simulations that have yet to be emulated with the more computationally complex BCS. As the expenses of computing resources continue to decline, however, it seems likely that the BCS/FCS will be more widely implemented and will start to realize the more advanced simulation results of which it is theoretically capable.

Small-scale facilitation and interference. The smallscale facilitation effected by small dots and lines in localizing contours suggests that these features may provide unoriented contributions to the oriented completion mechanisms. That is, it suggests that features which contain little or no orientational information may contribute to completion filter activity irrespective of standard, orientationdependent weighting factors. Since the edge and line-end detectors used to detect the inducing elements may be of a scale too large to respond to small anchors, these contributions may arise from detectors tuned to smaller scales. In the FACADE theory of perception (Grossberg, 1994), each bipole filter receives contributions from multiple scales. 
Orientational activity at large scales serves to establish the general boundary position, while small-scale activity serves to refine the position, especially in regions of high curvature. This mechanism may also explain small-scale facilitation effects of short lines and dots. Unoriented stimuli, such as dots, can be expected to excite cells of all orientations at some small scales, thus providing an "unoriented" contribution for inclusion in completion filters of all orientations. It is important that such small-scale components not be able to trigger completion filters by themselves-a series of dots (as in Figure 3b) does not induce illusory contours - but merely bias the position of completion initiated by larger scale inducers. In systems containing feedback loops, such as the BCS/FCS and the model of Finkel and Sajda (1992), a small bias is sufficient to induce robust illusory contours as reentrant activity reinforces the initial bias.

The converse effect of facilitation - the interference effect of small details near inducing elements-may result from a combination of factors. The fundamental difference between facilitating and interfering elements is the spatial proximity of the element to the inducers, and to the illusory contour itself. A facilitating element does not lie in the immediate area of any inducer but does lie on or near the illusory contour. Conversely, an interfering element lies in the immediate area of the inducer but does not lie on the illusory contour. In defining mechanisms by which these opposite effects can be captured, one must concentrate on these basic dissimilarities.

In modeling interference effects, one possibility is that the elements are simply frustrating the filters being used for edge and line-end detection, altering the spatial luminance distribution and thus leading to degraded or spuriously tilted responses that could weaken completion strengths. Given the small scale of the interfering elements, however, this proposition is dubious. A more plausible hypothesis is that these elements are providing small-scale orientational information that conflicts with orientational information at larger scales. For example, in Kennedy's (1988) demonstrations of the effect of line-end shape, large-scale information about the orientation of the whole line is contradicted (at the line ends) by smaller scale information about the shape of the line end. Cross-orientation, cross-scale competition might explain the interference effect, as small-scale cell responses inhibit responses at larger scales at the same or nearby positions. As described above in the context of facilitation, unoriented interfering elements would provide tonic inhibition at all orientations, while oriented elements would provide more selective inhibition. Competitive interactions between scales within the FACADE system cause the optimal scale at each position to inhibit weaker scales, allowing fine details and regions of high curvature to be perceived veridically. The combination of this mechanism, absent from all other models of contour formation, and interorientation competition yields a system consistent with small-scale interference effects. Since competition is spatially localized, this mechanism does not preclude facilitation effects of elements not in the immediate area of the inducers. It is unclear whether interscale cellular interactions should be unidirec- tional-only inhibition from small scales to larger scales or whether large scale to smaller scale interactions should also be implemented. However, the current psychophysical data seem to indicate an asymmetric relation.

Inducer structure and figural interference. The failure of thin lines to induce collinear illusory contours requires that parallel completion only be initiated when edge inducers have sufficient thickness. A simple method to accomplish this is to disallow completion when such a completion would lead to conflicting orientations or orientational cues at the same position. Thus a line could not be extended collinearly through a line end-an orientational cue for an occluding object perpendicular to the line itself. The concept of "spatial impenetrability" in the BCS accomplishes this function by allowing perpendicular orientations to inhibit bipole filter activity, canceling out parallel completion. Although the CMNCP does have a mechanism to suppress the presence of multiple orientations at the same position after grouping, it apparently maintains no mechanism that would disallow parallel completion with thin line inducers: the cornerness parameter $\kappa$ prohibits line ends from participating in para completion but does not prevent central line regions from contributing. To rectify this shortcoming, end-stopped cells would need to inhibit para completion in a fashion analogous to that employed by the spatial impenetrability mechanisms of the BCS.

Figural interference, in which lines crossing an illusory contour destroy that contour, can be handled with a mechanism similar to that used to manage the effect of inducer structure, forcing near-perpendicular orientation information to inhibit completion. Spatial impenetrability allows the BCS to model figural interference; the CMNCP contains no suitable mechanism. The combination of spatial impenetrability and the feedback loop of the BCS also serves to eliminate illusory contours with different orientations but with the same position. These mechanisms force the system to choose between conflicting illusory percepts and thus is essential to reproducing the psychophysical findings regarding the perception of ambiguous illusory figures.

Sophisticated mechanisms for segregating boundaries and surfaces into multiple depth planes, as in Finkel and Sajda's (1992) model and Grossberg's (1994) FACADE system, may also play significant roles in explaining the effects of both inducer structure and figural interference, since these determinants are dependent on the perceived depth of each scenic element. For example, although modal collinear completion of thin lines is precluded when the lines appear in the foreground, amodal collinear completion should be allowed when the lines are perceived in the background. Both the intermediate level vision models mentioned above support this kind of depth-dependent completion.

\section{High-Level Determinants}

Until very recently, neural models have all but ignored high-level determinants. Even now, most of the high-level determinants discussed earlier are well beyond the scope 
of current systems, and, at the level of detailed neural modeling, promise to remain so for the near future. We simply have insufficient knowledge of the dynamics of cognition to model such cognitive determinants as the interactions between memory, perceptual set, and illusory figure perception. An incomplete understanding of the underlying cognitive processes does not, however, preclude us from modeling how the results of these processes influence illusory contour formation.

Depth modifications. The one high-level determinant that has been the topic of substantial modeling research is the effect of depth modifications. As mentioned earlier, determination of the surface to which each contour belongs is the crucial step in determining how these surfaces occlude one another (Nakayama et al., 1990). This form of depth information, necessarily based solely on real, luminance-defined contours, can be propagated to create a depth ordering of both real and illusory contours (Finkel \& Sajda, 1992, 1994; Grossberg, 1994; Heitger \& von der Heydt, 1993; Sajda \& Finkel, 1992a, 1992b, 1995). Kellman and Shipley (1991) have posited that both modal (illusory contour) and amodal completion are subtended by the same mechanism, with depth ordering determining which contours are perceived as modal. This hypothesis has been supported by recent data indicating that certain low-level determinants have equivalent effects on illusory contour and amodal contour perception (Shipley \& Kellman, 1992a). Like Kellman and Shipley's model, Grossberg's (1994) FACADE system employs a depth stratification scheme with a single completion mechanism for both modal and amodal completion, but it also includes a definite mechanism for determination of which contour should be visible - the filling in of the FCS. The FACADE system bases initial depth ordering primarily on binocular cues, but many depth-related illusory contour effects involve purely monocular stimuli. This system must be extended to include depth-ordering schemes based on monocular cues, such as the local cues exploited by Heitger and von der Heydt (1993) and Sajda and Finkel (1992a, 1992b, 1995; Finkel \& Sajda, 1994): line ends and corners as occlusion indicators, local concavities, similarity and proximity of figural elements, and figure closure.

An important aspect of depth-ordering formalisms is that they explain how changes in the perception of depth ordering can facilitate illusory contour formation in the presence of (what would normally be) interfering elements, such as the overlayed textural elements of Rock and Anson (1979) (Figure 16). Such systems can also explain how flips in the perceptual depth ordering of ambiguous illusory contours can lead to instantiation of different sets of illusory contours. Determination of illusory contour strength depends not only on the relative stratification of illusory figure and inducing elements, but is also affected by the absolute depths; as illusory figures are stereoscopically forced closer to the observer, contour strength increases. It is unknown whether increasing depth via monocular cues leads to the same effect. Reconciling depth effects with neural models will require mechanisms to mediate contour strength by both relative stratifications--is the il- lusory figure in front of, or behind, the inducers?--and by absolute stereoscopic information-how far in front of (or behind) the inducers is the illusory figure? Research into the modeling and psychophysics of the effects of depth ordering on illusory contour formation represents the next major step in advancing neural systems.

Cognitive factors. Cognitive factors are more difficult to integrate into neural models than depth effects, primarily because we have only a vague understanding of the underlying processes themselves; let alone their neural substrates. Presumably, as our knowledge base becomes more complete, determinants with progressively higher loci of origin will be incorporated into neural systems. For now, however, neural models of illusory contour formation are left with fundamental limitations on the modeling of many high-level determinants. This is not to say that the effects of cognitive factors have been - or should be - completely ignored by neural modelers, although coverage to date has been undeniably sparse. Until neural systems directly address the daunting problems of incorporating the various cognitive determinants, they cannot truly be considered general models of the contour formation process.

As mentioned during the discussion of inducer completeness, some high-level determinants may not be as inherently cognitive as initially thought. Certain effects of the seemingly cognitive factor of inducer completeness may be closely related to local mechanisms (Albert, 1993; Sajda \& Finkel, 1995). Further psychophysical quantification of high-level determinants, where possible, may reveal additional low-level determinants. However, it is doubtful that the complex roles of memory and perceptual set in illusory contour generation will be demoted to simpler explanations.

In presenting their models of contour formation, researchers almost invariably mention top-down mediation of contour strength by "higher level" processes. Grossberg and Mingolla (1985a, 1985b, 1987a, 1987b; Grossberg, $1987 \mathrm{a}, 1987 \mathrm{~b}, 1994)$ have been particularly meticulous in stressing the importance of these top-down effects, proposing that the BCS/FCS interacts with an object recognition system (ORS) responsible for contour reinforcement, attentional effects, and priming, as well as for primarily feedforward tasks such as object recognition and image understanding. The BCS/FCS $\leftrightarrow$ ORS interactions have not yet been simulated; but they appear to be theoretically sound, and they are sufficiently well specified for attempts at qualitative simulation of some of the simpler high-level determinants.

The most promising means of incorporating cognitive factors is through the use of top-down priming. A look at any of the figures included in this paper makes it clear that illusory contours never achieve the perceptual salience of real luminance edges. Some observers may not perceive every contour, and for most observers, foveation on a contour will weaken or eliminate the contour (Coren, 1972). Perhaps, then, illusory contours tend to lie near the threshold of perception, as is indicated by the attention studies of Gurnsey et al. (1992). Slight indications from cognitive processes may suppress or strengthen contours, or alter 
their paths completely. For a perceptual set process, a topdown prime might be something like "pay particular attention to edge alignments" (as in Rock \& Anson, 1979) or "there might be corners at these positions" (as in Coren et al., 1986). The alternative scheme-that of cognitive processes sorting through a web of tentative contours, supplied by neural mechanisms, to arrive at a single percept is also possible, but it lacks the economy and elegance of a system that reuses a single mechanism to both generate and refine illusory contours. Neural models that already include feedback, such as the BCS/FCS, FACADE, and Finkel and Sajda's (1992) system, are particularly amenable to priming; the slightest top-down hint about which features might be important to contour formation or where to look for an illusory contour could be propagated by the feedback mechanisms to completely reconfigure the percept. Indeed, Sajda and Finkel (1995) have proposed a clever paradigm that essentially hypothesizes contours based on secondary cues, which are implicitly verified only when contours generated by the feedback process trigger primary cues.

The exact form that top-down primes might take will depend on the particular neural model, but likely candidates include attentional mechanisms that raise the model's sensitivity within specific regions, direct excitation (or inhibition) of oriented or end-stopped cells, and global biasing of particular model parameters. An effective system will probably need to accommodate multiple types of primes. Although it would be more satisfying to model the cognitive processes as well, we can assign generation of the prime or attentional determinant to a cognitive daemon without compromising the neural models, because these higher level processes are at least partially independent of the modeling of illusory contour formation. With this technique, the explanatory power of next-generation models should extend well into the domain of high-level determinants.

\section{SUMMARY}

Twenty years of debate over the nature and dynamics of illusory contour formation have gradually resolved to a determined focus on neural models. Qualitative studies designed to yield insight into the general form of the underlying process-cognitive or retinal, gestalt or geometric-have for the most part given way to quantitative studies formulated in order to tabulate the specific determinants of illusory contour strength, illusory brightening, and perceived depth of the illusory figure. Recognition that neural systems represent, at least to a rough approximation, the substrate of illusory contour formation has led to recent experiments designed to refine our understanding of neural models and constrain the possible types of interactions and mechanisms. A thorough understanding of the structure of models like the BCS and CMNCP, and of other innovative modeling ideas, is a necessary prerequisite for continued development of psychophysical and neurophysiological experiments that will advance the sophistication and accuracy of these systems. Issues of scale interactions, the relation between edges and line ends, the effects of line spacing, and the effects of depth ordering are just some of the areas in which additional study is required.

The evaluation of current neural models provided in the previous section indicates the vast degree to which improvements are possible. Not even the simplest of low-level determinants have been quantitatively modeled, and only recently have simulations of qualitative effects been published. Further evaluations of the BCS, CMNCP, and other neural models will require that they progress from theory to demonstration. At the same time, these models must be reconciled with low-level determinants that they cannot presently emulate, by extension or modification. In particular, interscale interactions are conspicuously absent from current models, preventing the emulation of smallscale facilitation and interference effects. As quantitative simulations appear and neural models are committed to a single parameter set, more psychophysically testable predictions will be possible. All models, with the notable exception of the BCS/FCS, must be extended to rectify an even more glaring omission: the lack of mechanisms to simulate brightness effects. Although the amount of psychophysical illusory brightness data exceeds that concerning illusory contour strength and depth effects, there have been no qualitative BCS/FCS simulations of the effect of low-level inducer variations on brightness - an area in which this model appears particularly well qualified.

Of the many high-level determinants, the pronounced effects of depth ordering on contour strength are most amenable to detailed neural modeling. Although simple depth-ordering mechanisms have found their way into some current models (such as the CMNCP), much more sophisticated methods will be required in order to reproduce psychophysical results. Such methods have recently been formulated (Grossberg, 1994; Kellman \& Shipley, 1991; Sajda \& Finkel, 1995), but further development is still required, as are more extensive simulations. Purely cognitive factors, such as the effects of memory and perceptual set, are difficult to model explicitly, but integration of these determinants into neural models may be possible through the use of top-down primes to represent the outcome of cognitive processes. The overdue incorporation of these cognitive primes represents an intriguing and promising area for future research. Finally, we must be aware that a model of monocular, luminance-defined illusory contour formation must fit within the larger framework of illusory contour formation in other domains, which in turn must represent a subsystem of comprehensive human vision. To this end, the incorporation and integration of contour formation systems into models of other visual domains must represent the ultimate goal of our research.

\section{REFERENCES}

Abravanet, E. (1982). Perceiving subjective contours during early childhood. Journal of Experimental Child Psychology, 33, 280-287. ALberT, M. K. (1993). Parallelism and the perception of illusory contours. Perception, 22, 589-595.

Albert, M. K., \& Hoffman, D. D. (1992). The generic viewpoint as- 
sumption and illusory contours [Abstract]. Investigative Ophthalmology \& Visual Science, 33, 68.

ANDERsen, G. J., \& CoRTESE, J. M. (1989). 2-D contour perception resulting from kinetic occlusion. Perception \& Psychophysics, 46, 49-55.

Banton, T., \& LeVI, D. M. (1992). The perceived strength of illusory contours. Perception \& Psychophysics, 52, 676-684.

Barlow, H. B., \& Hill, R. M. (1963). Evidence for a physiological explanation of the waterfall phenomena and figural aftereffects. Nature, 200, 1345-1347.

BECKer, M. F., \& KNOPP, J. (1978). Processing of visual illusions in the frequency and spatial domains. Perception \& Psychophysics, 23, 521 526.

Berkley, M. A., Debruyn, B., \& Orban, G. (1994). Illusory, motion, and luminance-defined contours interact in the human visual system. Vision Research, 34, 209-216.

Bertenthal, B. I., Campos, J. J., \& Haith, M. M. (1980). Development of visual organization: The perception of subjective contours. Child Development, 51, 1072-1080.

BRADLEY, D. R. (1982). Binocular rivalry of real vs. subjective contours Perception \& Psychophysics, 32, 85-87.

BRADLEY, D. R. (1987). Cognitive contours and perceptual organization In S. Petry \& G, E. Meyer (Eds.), The perception of illusory contours (pp. 201-212). New York: Springer-Verlag.

Bradley, D. R., \& Dumais, S. T. (1975). Ambiguous cognitive contours. Nature, 257, 582-584.

Bradley, D. R., \& DumaIs, S. T. (1984). The effects of illumination level and retinal size on the depth stratification of subjective contour figures. Perception, 13, 155-164.

Bradley, D. R., Dumais, S. T., \& PeTry, H. M. (1976). Reply to Cavonius. Nature, 261, 77-78.

Bradley, D. R., \& MATES, S. M. (1985). Perceptual organization and apparent brightness in subjective contour figures. Perception, 14, 645-653.

Bradley, D. R., \& PeTRY, H. M. (1977). Organizational determinants of subjective contour: The subjective Necker cube. American Journal of Psychology, 90, 253-262.

BRADY, M., \& GRIMSON, W. E. L. (1981). The perception of subjective surfaces (A.I. Memo No. 666). Cambridge, MA: MIT Artificial Intelligence Laboratory.

Bravo, M., Blake, R., \& Morrison, S. (1988). Cats see subjective contours. Vision Research, 28, 861-865.

BRESSAN, P. (1987). Subjective rarefaction in illusory figures: The inadequacy of apparent lightness as an explanation. Perception, 16, 461-466.

Bressan, P., \& Vallortigara, G. (1986). Subjective contours can produce stereokinetic effects. Perception, 15, 409-412.

Bressan, P., \& Vallortigara, G. (1987). Stereokinesis with moving visual phantoms. Perception, 16, 73-78.

Bressan, P., \& Vallortigara, G. (1991). Illusory depth from moving subjective figures and neon colour spreading. Perception, 20, 637-644.

BRIGNER, W. L. (1982). Mathematical model for the filling-in of illusionary contour. Perceptual \& Motor Skills, 54, 815-820.

Brigner, W. L., \& Gallagher, M. B. (1974). Subjective contour: Apparent depth or simultaneous brightness contrast? Perceptual \& Motor Skills, 38, 1047-1053.

Bross, M., \& Michel ANGELI, L. (1988). The role of inducer alignment and practice in the perception of subjective contours. Perception, 17, 391

Bruno, N., \& BeRTAMINI, M. (1990). Identifying contours from occlusion events. Perception \& Psychophysics, 48, 331-342.

Bruno, N., \& Gerbino, W. (1987). Amodal completion and illusory figures: An information-processing analysis. In S. Petry \& G. E. Meyer (Eds.), The perception of illusory contours (pp. 220-223). New York: Springer-Verlag.

Bruno, N., \& Gerbino, W. (1991). Illusory figures based on local kinematics. Perception, 20, 259-274.

Brussel, E. M., STober, S. R., \& Bodinger, D. M. (1977). Sensory information and the subjective contour. American Journal of Psychology, 90, 145-156.

COREN, S. (1972). Subjective contours and apparent depth. Psychological Review, 79, 359-367.

COREN, S. (1991). Retinal mechanisms in the perception of subjective contours: The contribution of lateral inhibition. Perception, 20, 181-191.

COREN, S., \& PORAC, C. (1983). Subjective contours and apparent depth: A direct test. Perception \& Psychophysics, 33, 197-200.
Coren, S., Porac, C., \& Theodor, L. H. (1986). The effects of perceptual set on the shape and apparent depth of subjective contours. Perception \& Psychophysics, 39, 327-333.

Coren, S., Porac, C., \& Theodor, L. H. (1987). Set and subjective contour. In S. Petry \& G. E. Meyer (Eds.), The perception of illusory contours (pp. 237-245). New York: Springer-Verlag.

Coren, S., \& Theodor, L. H. (1975). Subjective contour: The inadequacy of brightness contrast as an explanation. Bulletin of the Psychonomic Society, 6, 87-89.

Davi, M., Pinna, B., \& Sambin, M. (1992). Amodal completion versus induced inhomogeneities in the organization of illusory figures. Perception, 21, 627-636.

DAY, R. H. (1986). Enhancement of edges by contrast, depth and figure: The origin of illusory contours. In J. D. Pettigrew, K. J. Sanderson, \& W. R. Levick (Eds.), Visual neuroscience (pp. 352-364). Cambridge: Cambridge University Press.

DAY, R. H. (1987). Cues for edges and the origin of illusory contours: An alternative approach. In S. Petry \& G. E. Meyer (Eds.), The perception of illusory contours (pp. 53-61). New York: Springer-Verlag.

DAY, R. H., DICKINSON, R. G., \& JORY, M. K. (1977). The Poggendorff illusion with subjective contours. Quarterly Journal of Experimental Psychology, 29, 219-226.

DAY, R. H., \& JORY, M. K. (1980). A note on a second stage in the formation of illusory contours. Perception \& Psychophysics, 27, 89-91.

DAY, R. H., \& KASPERCZYK, R. T. (1983). Amodal completion as a basis for illusory contours. Perception \& Psychophysics, 33, 355-364.

de Weerd, P., Vandenbusche, E., de Bruyn, B., \& Orban, G. A. (1990). Illusory contour orientation discrimination in the cat. Behavioural Brain Research, 39, 1-17.

DE WEERT, C. M. M. (1983). The role of colours in the formation of subjective contours. Psychological Research, 45, 117-134.

Dobrins, A., Zucker, S. W., \& Cynader, M. S. (1989). Endstopping and curvature. Vision Research, 29, 1371-1387.

DRESP, B. (1992). Local brightness mechanisms sketch out surfaces but do not fill them in: Psychophysical evidence in the Kanizsa square. Perception \& Psychophysics, 52, 562-570.

DRESP, B. (1993). Bright lines and edges facilitate the detection of small light targets. Spatial Vision, 7, 213-225.

DRESP, B., \& BonNET, C. (1991). Psychophysical evidence for low-level processing of illusory contours and surfaces in the Kanizsa square. $\mathrm{Vi}$ sion Research, 31, 1813-1817.

DRESP, B., LoRenceau, J., \& Bonnet, C. (1990). Apparent brightness enhancement in the Kanizsa square with and without illusory contour formation. Perception, 19, 483-489.

Dumais, S. T., \& Bradley, D. R. (1976). The effects of illumination level and retinal size on the apparent strength of subjective contours. Perception \& Psychophysics, 19, 339-345.

EhrensteIN, W. (1941). Über Abwandlungen der L. Hermannschen Helligkeits-Erscheinung. Zeitschrift für Psychologie, 150, 83-91.

EJIMA, Y., \& TAKAHASHI, S. (1988). Illusory contours induced by isoluminant chromatic patterns. Vision Research, 28, 1367-1377.

Fahle, M., \& PALM, G. (1991). Perceptual rivalry between illusory and real contours. Biological Cybernetics, 66, 1-8.

FIELD, D. J., HAYES, A., \& HESS, R. F. (1992). Contour integration by the human visual system: Evidence for a local "association field." Vision Research, 33, 173-193.

Finkel, L. H., \& EDElman, G. M. (1989). Integration of distributed cortical systems by reentry: A computer simulation of interactive functionally segregated visual areas. Journal of Neuroscience, 9, 3188-3208.

FinkEL, L. H., \& SAJDA, P. (1992). Object discrimination based on depth-from-occlusion. Neural Computation, 4, 901-921.

FinKel, L. H., \& SAJDA, P. (1994). Constructing visual perception. American Scientist, 82, 224-237.

Francis, G., Grossberg, S., \& Mingolla, E. (1994). Cortical dynamics of feature binding and reset: Control of visual persistence. Vision Research, 34, 1089-1104.

Frisby, J. P., \& Clatworthy, J. L. (1975). Illusory contours: Curious cases of simultaneous brightness contrast. Perception, 4, 349-357.

Gellatly, A. R. H. (1980). Perception of an illusory triangle with masked inducing figure. Perception, 9, 599-602.

Gellatly, A. R. H. (1982). Perceptual leaming of illusory contours and colour. Perception, 11, 655-661. 
Gellatly, A. R. H., \& Bishop, M. J. (1987). The perception of illusory contours: A skills analysis. In S. Petry \& G. E. Meyer (Eds.), The perception of illusory contours (pp. 262-267). New York: SpringerVerlag.

Gerbino, W., \& Kanizsa, G. (1987). Can we see constructs? In S. Petry $\&$ G. E. Meyer (Eds.), The perception of illusory contours (pp. 246252). New York: Springer-Verlag.

GillaM, B. (1987). Perceptual grouping and subjective contours. In S. Petry \& G. E. Meyer (Eds.), The perception of illusory contours (pp. 268-273). New York: Springer-Verlag.

Gillam, B. J., \& Goodenough, B. (1994). Subjective contours at line terminators-the effect of the relationship between terminator arrangement and line arrangement [Abstract]. Investigative Ophthalmology \& Visual Science, 35, 1627.

GiNSBURG, A. P. (1975). Is the illusory triangle physical or imaginary? Nature, 257, 219-220.

GinsBurg, A. P. (1987). The relationship between spatial filtering and subjective contours. In S. Petry \& G. E. Meyer (Eds.), The perception of illusory contours (pp. 126-130). New York: Springer-Verlag.

Goldstein, M. B., \& WeinTraub, D. J. (1972). The parallel-less Poggendorff: Virtual contours put the illusion down but not out. Perception \& Psychophysics, 11, 353-355.

Gove, A. (1993). A neural network model of visual segmentation: Illusory contour formation, brightness induction, and grouping of scenic elements. Unpublished doctoral dissertation, Boston University.

Gove, A., Grossberg, S., \& Mingolla, E. (1994a). Brightness perception, illusory contours, and binocular corticogeniculate feedback (Tech. Rep. No. CAS/CNS-TR-94-033). Boston University, Department of Cognitive and Neural Systems.

Gove, A., Grossberg, S., \& Mingolla, E. (1994b). A link between brightness perception, illusory contours, and binocular corticogeniculate feedback [Abstract]. Investigative Ophthalmology \& Visual Science, 35, 1437.

Grabowecky, M., \& Treisman, A. (1989). Attention and fixation in subjective contour perception [Abstract]. Investigative Ophthalmology \& Visual Science, 30, 457.

GREGORY, R. L. (1972). Cognitive contours. Nature, 238, 51-52.

GREGORY, R. L. (1977). Vision with isoluminant color contrast: 1. A projection technique and observations. Perception, 6, 113-119.

GREGORY, R. L. (1987). Illusory contours and occluding surfaces. In S. Petry \& G. E. Meyer (Eds.), The perception of illusory contours (pp. 81-89). New York: Springer-Verlag.

GREGORY, R. L., \& HARRIS, J. P. (1974). Illusory contours and stereo depth. Perception \& Psychophysics, 15, 411-416.

Grosof, D. H., ShaPLEy, R. M., \& HAWKEN, M. J. (1993). Macaque V1 neurons can signal "illusory" contours. Nature, $365,550-552$.

GrossBerG, S. (1987a). Cortical dynamics of three-dimensional form, color, and brightness perception: II. Binocular theory. Perception \& Psychophysics, 41, 117-158.

GrossberG, S. (1987b). Cortical dynamics of three-dimensional form, color, and brightness perception: 1. Monocular theory. Perception \& Psychophysics, 41, 87-116.

GROSSBERG, S. (1994). 3-D vision and figure-ground separation by visual cortex. Perception \& Psychophysics, 55, 48-121.

GrossBerg, S., \& Marshall, J. (1989). Stereo boundary fusion by cortical complex cells: A system of maps, filters, and feedback networks for multiplexing distributed data. Neural Networks, 2, 29-51.

Grossberg, S., \& Mingolla, E. (1985a). Neural dynamics of form perception: Boundary completion, illusory figures, and neon color spreading. Psychological Review, 92, 173-211.

Grossberg, S., \& Mingolla, E. (1985b). Neural dynamics of perceptual grouping: Textures, boundaries, and emergent segmentations Perception \& Psychophysics, 38, 141-171.

Grossberg, S., \& Mingolla, E. (1987a). Neural dynamics of surface perception: Boundary webs, illuminants, and shape from shading. Computer Vision, Graphics, \& Image Processing, 37, 116-165.

Grossberg, S., \& Mingolla, E. (1987b). The role of illusory contours in visual segmentation. In S. Petry \& G. E. Meyer (Eds.), The perception of illusory contours (pp. 116-125). New York: Springer-Verlag.

Grossberg, S., Mingolla, E., \& Williamson, J. R. (1994). Synthetic aperture radar processing by a multiple scale neural system for bound- ary surface representation (Tech. Rep. No. CAS/CNS-TR-94-001) Boston University, Department of Cognitive and Neural Systems Grossberg, S., \& RUDD, M. E. (1989). A neural architecture for visual motion perception: Group and element apparent motion. Neural Networks, 2, 421-450.

GRossBERG, S., \& RuDD, M. E. (1992). Cortical dynamics of visual motion perception: Short-range and long-range apparent motion. Psychological Review, 99, 78-121.

GrossBerg, S., \& Todorović, D. (1988). Neural dynamics of 1-D and 2-D brightness perception: A unified model of classical and recent phenomena. Perception \& Psychophysics, 43, 241-277.

GuRnsey, R., Humphrey, G. K., \& Kapitan, P. (1992). Parallel discrimination of subjective contours defined by offset gratings. Perception \& Psychophysics, 52, 263-276.

HALPERN, D. F. (1981). The determinants of illusory-contours perception. Perception, 10, 199-213.

HALPERN, D. F. (1987). The functional equivalence of objective and illusory brightness enhancement. In S. Petry \& G. E. Meyer (Eds.), The perception of illusory contours (pp. 171-175). New York: Springer-Verlag

HALPERN, D. F., \& SALZMAN, B. (1983). The multiple determination of illusory contours: 1. A review. Perception, 12, 281-291.

Halpern, D. F., Salzman, B., Harrison, W., \& Widaman, K. (1983) The multiple determination of illusory contours: 2 . An empirical investigation. Perception, 12, 293-303.

HALPERN, D. F., \& WARM, J. S. (1980). The disappearance of real and subjective contours. Perception \& Psychophysics, 28, 229-235.

HALPERN, D. F., \& WARM, J. S. (1984). The disappearance of dichoptically presented real and subjective contours. Bulletin of the Psychonomic Society, 22, 433-466.

HAMSHER, K. D. (1978). Stereopsis and the perception of anomalous contours. Neuropsychologia, 16, 453-459.

Heitger, F., Rosenthaler, L., von der Heydt, R., Peterhans, E., \& KÜBLER, O. (1992). Simulation of neural contour mechanisms: From simple to end-stopped cells. Vision Research, 32, 963-978.

HEITGER, F., \& vON DER HEYDT, R. (1993). A computational model of neural contour processing: Figure-ground segregation and illusory contours. In IEEE 4th International Conference on Computer Vision (pp. 32-40). Los Alamitos, CA: IEEE Computer Society Press.

HERSHBERGER, W., \& STALLARD, S. (1984). Contrast variability lightens subjective figures. Perception \& Psychophysics, 36, 92-94.

JORY, M. K. (1987). Increment threshold in illusory contour line pattern. In S. Petry \& G. E. Meyer (Eds.), The perception of illusory contours (pp. 183-190). New York: Springer-Verlag.

JORY, M. K., \& DAY, R. H. (1979). The relationship between brightness contrast and illusory contours. Perception, 8, 3-9.

JULESZ, B. (1960). Binocular depth perception of computer-generated patterns. Bell Systems Technical Journal, 39, 1125-1162.

JulESZ, B., \& FrISBY, J. P. (1975). Some new subjective contours in random-line stereograms. Perception, 4, 145-150.

JUNG, R., \& SPILlman, L. (1970). Receptive-field estimation and perceptual integration in human vision. In F. A. Young \& D. B. Lindsley (Eds.), Early experience and visual information processing in perceptual and reading disorders (pp. 181-197). Washington, DC: National Academy of Sciences.

KANIZSA, G. (1955). Margini quasi-percettivi in campi con stimulazione omogenea. Rivista di Psicologia, 49, 7-30.

KANIZSA, G. (1974). Contours without gradients or cognitive contours? Italian Journal of Psychology, 1, 107-123.

Kanizsa, G. (1976, April). Subjective contours. Scientific American 234, 48-52.

Kanizsa, G. (1979). Organization in vision: Essays on Gestalt perception. New York: Praeger

KANIZSA, G. (1987). 1986 Addendum [to translated article]. In S. Petry \& G. E. Meyer (Eds.), The perception of illusory contours (p. 49). New York: Springer-Verlag.

KaPlaN, G. A. (1969). Kinetic disruption of optical texture: The perception of depth at an edge. Perception \& Psychophysics, 6, 193-198.

Kellman, P. J., \& LoukIDES, M. (1987). An object perception approach to static and kinetic subjective contours. In S. Petry \& G. E. Meyer (Eds.), The perception of illusory contours (pp. 151-164). New York: Springer-Verlag. 
Kellman, P. J \& Shipley, T. F. (1991). A theory of visual interpolation in object perception. Cognitive Psychology, 23, 141-221.

KenNEDY, J. M. (1976). Sun figure: An illusory diffuse contour resulting from an arrangement of dots. Perception, 5, 479-481.

KeNNEDY, J. M. (1978a). Illusory contours and the ends of lines. Perception, 7, 605-607.

KENNEDY, J. M. (1978b). Illusory contours not due to completion. Perception, 7, 187-189.

KENNEDY, J. M. (1979). Subjective contours, contrast, and assimilation In C. F. Nodine \& D. F. Fisher (Eds.), Perception and pictorial representation (pp. 167-195). New York: Praeger.

KENNEDY, J. M. (1981). Illusory brightness and the ends of petals: Change in brightness without aid of stratification or assimilation effects. Perception, 10, 583-585.

KENNEDY, J. M. (1987). Lo, perception abhors not a contradiction. In S. Petry \& G. E. Meyer (Eds.), The perception of illusory contours (pp. 253-261). New York: Springer-Verlag.

KeNNEDY, J. M. (1988). Line endings and subjective contours. Spatial Vision, 3, 151-158.

KENNEDY, J. M., \& LEE, H. (1976). A figure-density hypothesis and illusory contour brightness. Perception, 5, 387-392.

KLYMENKo, V., \& WeissteIN, N. (1981). The motion-induced contour Perception, 10, 627-636.

KLymenko, V., \& Weisstein, N. (1983). The edge of an event: Invariants of a moving illusory contour. Perception \& Psychophysics, 34, 140-148.

Klymenko, V., \& Weisstein, N. (1984). The razor's edge: A dichotomy between monohedral and dihedral edge perception. Vision Research, 24, 995-1002.

Klymenko, V., \& Weisstein, N. (1987). The resonance theory of kinetic shape perception and the motion-induced contour. In S. Petry \& G. E. Meyer (Eds.), The perception of illusory contours (pp. 143148). New York: Springer-Verlag.

Klymenko, V., Weisstein, N., \& Ralston, J. V. (1987). Illusory contours, projective transformations and kinetic shape perception. Acta Psychologica, 64, 229-243.

LANDAUER, A. A. (1978). Subjective states and the perception of subjective contours. In J. P. Sutcliff (Ed.), Conceptual analysis and method in psychology: Essays in honour of W. M. O'Neil (pp. 142-146). Sydney: University Press.

Lawson, R. B., CoWan, E., GibBs, T. D., \& Whitmore, C. G. (1974), Stereoscopic enhancement and erasure of subjective contours. Journal of Experimental Psychology, 103, 1142-1146.

LESHER, G. W. (1993). Neural networks for vision and pattern recognition: Boundary completion, spatial mapping, and multidimensional data fusion. Unpublished doctoral dissertation, Boston University.

Lesher, G. W., Grossberg, S., \& Mingolla, E. (1994). Shared mechanisms for hyperacuity and illusory contours [Abstract]. Investigative Ophthalmology \& Visual Science, 35, 2008.

Lesher, G. W., \& Mingolla, E. (1993). The role of edges and line ends in illusory contour formation. Vision Research, 33, 2253-2270.

Livingstone, M., \& Hubel, D. (1988, May 6). Segregation of form, color, movement, and depth: Anatomy, physiology, and perception. Science, 240, 740-749.

MacKinnon, G. E., Forde, J., \& Piggins, D. J. (1969). Stabilized images, steadily fixated figures, and prolonged after-images. Canadian Journal of Psychology, 23, 184-195.

MAGUiRE, W. M., \& BROWN, J. M. (1987). The current state of research into visual phantoms. In S. Petry \& G. E. Meyer (Eds.), The perception of illusory contours (pp. 213-219). New York: Springer-Verlag.

Manjunath, B. S., \& ChellapPa, R. (1993). A unified approach to boundary perception: Edges, textures, and illusory contours. IEEE Transactions on Neural Networks, 4, 96-108.

MAST, R., \& Fox, R. (1994). Support ratios and subjective contour perception [Abstract]. Investigative Ophthalmology \& Visual Science, 35, 2009

MATHER, G. (1988). Temporal properties of apparent motion in subjective figures. Perception, 17, 729-736.

MEYer, G. E., \& DougherTy, T. (1987). Effects of flicker-induced depth on chromatic subjective contours. Journal of Experimental Psychology: Human Perception \& Performance, 13, 353-360.

MEYER, G. E., \& FISH, D. (1987). Illusory contours, texture segregation, and a configural inferiority effect. In S. Petry \& G. E. Meyer (Eds.), The perception of illusory contours (pp. 224-234). New York: SpringerVerlag.

Meyer, G. E., \& Garges, C. (1979). Subjective contours and the Poggendorff illusion. Perception \& Psychophysics, 26, 302-304.

MEYER, G. E., \& Ming, C. Y. (1988). The visible persistence of illusory contours. Canadian Journal of Psychology, 42, 479-488.

Meyer, G. E., \& Petry, S. (1987). Top-down and bottom-up: The illusory contour as a microcosm of issues in perception. In S. Petry \& G. E. Meyer (Eds.), The perception of illusory contours (pp. 3-20). New York: Springer-Verlag.

MinguzzI, G. F. (1987). Anomalous figures and the tendency to continuation. In S. Petry \& G. E. Meyer (Eds.), The perception of illusory contours (pp. 71-75). New York: Springer-Verlag.

Movshon, J. A., Chambers, B. E. I., \& Blakemore, C. (1972). Interocular transfer in normal humans, and those who lack stereopsis. Perception, 1, 483-490.

Mulvanny, P., Macarthur, R., \& Sekuler, R. (1982). Thresholds for seeing visual phantoms and moving gratings. Perception, 11, 35-46.

MustilLo, P., \& Fox, R. (1986). The perception of illusory contours in the hypercyclopean domain. Perception \& Psychophysics, 40, 362-363.

Nakayama, K., Shimojo, S., \& Ramachandran, V. S. (1990). Transparency: Its relation to depth, subjective contours, and neon color spreading. Perception, 19, 497-513.

Paradiso, M. A., Shimojo, S., \& Nakayama, K. (1989). Subjective contours, tilt aftereffects, and visual cortical organization. Vision $R e-$ search, 29, 1205-1213.

PARKs, T. E. (1980). The subjective brightness of illusory figures: Is stratification a factor? Perception, 9, 361-363.

PARKS, T. E. (1981). Subjective figures: An infrequent, but certainly not unprecedented, effect. Perception, 10, 589-590.

PARKS, T. E. (1983). Further difficulties for the filtered-components approach to illusory visual contours. Perception \& Psychophysics, 34, 190-192.

PARKS, T. E. (1984). Illusory figures: A (mostly) atheoretical review. Psychological Bulletin, 95, 282-300.

Parks, T. E. (1985). Apparent depth and texture differences in illusory figure patterns: A paradox resolved. Perception \& Psychophysics, 37, 568-570.

PARKS, T. E. (1986). Illusory figures, illusory objects, and real objects. Psychological Review, 93, 207-215.

PARKS, T. E. (1987). On the relative frequency of depth effects in real versus illusory figures. Perception \& Psychophysics, 42, 333-336.

PARKS, T. E., \& MARKs, W. (1983). Sharp-edged vs. diffuse illusory circles: The effects of varying luminance. Perception \& Psychophysics, 33, 172-176.

Parks, T. E., \& Pendergrass, L. (1982). On the filtered-components approach to illusory visual contours. Perception \& Psychophysics, 32, 491-493.

PARKS, T. E., \& RoCK, I. (1990). Illusory contours from pictorially threedimensional inducing elements. Perception, 19, 119-121.

PASTORE, N. (1971). Selective history of theories of visual perception: 1650-1950. New York: Oxford Univerity Press.

Pessoa, L., Mingolla, E., \& Neumann, H. (1994). A multi-scale network model of brightness perception (Tech. Rep. No. CAS/CNS-94017). Boston University, Department of Cognitive \& Neural Systems.

Peterhans, E., \& von DER HEYdT, R. (1989). Mechanisms of contour perception in monkey visual cortex: II. Contours bridging gaps. Journal of Neuroscience, 9, 1749-1763.

Peterhans, E., \& VON DER HEYDT, R. (1991). Elements of form perception in monkey prestriate cortex. In A. Gorea, Y. Fregnac, Z. Kapoula, \& J. Findlay (Eds.), Representations of vision-Trends and tacit assumptions in vision research (pp. 1-12). Cambridge: Cambridge University Press.

Peterhans, E., von der Heydt, R., \& Baumgartner, G. (1986). Neuronal responses to illusory contour stimuli reveal stages of visual cortical processing. In J. D. Pettigrew, K. J. Sanderson, \& W. R. Levick (Eds.), Visual neuroscience (pp. 343-351). Cambridge: Cambridge University Press.

Petersik, J. T. (1987). Dependence of apparent movement of a subjective figure on the perceptual fate of inducing elements. Perception, 16, 453-459. 
Peterson, M. A., \& Gibson, B. S. (1994). Object recognition contributions to figure-ground organization: Operations on outlines and subjective contours. Perception \& Psychophysics, 56, 551-564.

Petry, S., \& Gannon, R. (1987). Time, motion, and objectness in illusory contours. In S. Petry \& G. E. Meyer (Eds.), The perception of illusory contours (pp. 193-200). New York: Springer-Verlag.

Petry, S., Harbeck, A., Conway, J., \& Levey, J. (1983). Stimulus determinants of brightness and distinctness of subjective contours. Perception \& Psychophysics, 34, 169-174.

PetTer, G. (1956). Nuove ricerche sperimentali sulla totalizzazione percettiva. Rivista di Psicologia, 50, 213-227.

Pinna, B., \& Sambin, M. (1991). A dynamic model for anomalous figures: The shape of line-induced brightness modifications. Perception, 20, 219-232.

Pomerantz, J. R., Goldberg, D. M., Golder, P. S., \& Tetewski, S. (1981). Subjective contours can facilitate performance in a reactiontime task. Perception \& Psychophysics, 29, 605-611.

Porac, C. (1978). Depth in objective and subjective contour patterns. Bulletin of the Psychonomic Society, 11, 103-105.

PrazDNy, K. (1983). Illusory contours are not caused by simultaneous brightness contrast. Perception \& Psychophysics, 34, 403-404.

PRAZDNY, K. (1985). On the nature of inducing forms generating perceptions of illusory contours. Perception \& Psychophysics, 37, 237-242.

Prazdny, K. (1986). Illusory contours from inducers defined solely by spatiotemporal correlation. Perception \& Psychophysics, 39, 175-178.

Predebon, J. (1985). Illusory contours and size illusions. Bulletin of the Psychonomic Society, 23, 47-49.

Pritchard, W. S., \& WARM, J. S. (1983). Attentional processing and the subjective contour illusion. Journal of Experimental Psychology. General, 112, 145-175.

Purghé, F. (1988). The role of figural incompleteness and brightness contrast in the formation of anomalous surfaces. Perception, 17, 365

PURGHÉ, F. (1991). Is amodal completion necessary for the formation of illusory figures? Perception, 20, 623-636.

PURGHé, F. (1993). Illusory contours from pictorially three-dimensional inducing elements: Counterevidence for Parks and Rock's example Perception, 22, 809-818.

Purghé, F., \& Coren, S. (1992a). Amodal completion, depth, stratification, and illusory figures: A test of Kanizsa's explanation. Perception, 21, 325-335.

Purghé, F., \& Coren, S. (1992b). Subjective contours 1900-1990: Research trends and bibliography. Perception \& Psychophysics, 51, 291 304.

Purghé, F., \& KatSARAs, P. (1991). Figural conditions affecting the formation of anomalous surfaces: Overall configuration versus single stimulus part. Perception, 20, 193-206.

RAMACHANDRAN, V. S. (1986a). Capture of stereopsis and apparent motion by illusory contours. Perception \& Psychophysics, 39, 361-373.

RamachandRaN, V. S. (1986b). Capture of stereopsis by illusory contours: Reply to Prazdny. Nature, 324, 393-394.

RAMACHANDRAN, V. S. (1987). Visual perception of surfaces: A biological theory. In S. Petry \& G. E. Meyer (Eds.), The perception of illusory contours (pp. 93-108). New York: Springer-Verlag.

RamaChandran, V. S., \& Cavanagh, P. (1985). Subjective contours capture stereopsis. Nature, 317, 527-530.

Ramachandran, V. S., Ruskin, D., CobB, S., Rogers-RamachanDRAN, D., \& TYLER, C. W. (1994). On the perception of illusory contours. Vision Research, 34, 3145-3152.

RATLIFF, F. (1965). Mach bands: Quantitative studies on neural networks in the retina. New York: Holden-Day.

REDIES, C. (1989). Discontinuities along lines: Psychophysics and neurophysiology. Neuroscience \& Biobehavioral Reviews, 13, 17-22.

Redies, C., Crook, J. M., \& Creutzfeldt, O. D. (1986). Neuronal responses to borders with and without luminance gradients in cat visual cortex and dorsal lateral geniculate nucleus. Experimental Brain Research, 61, 469-481.

Redies, C., \& SpIllmaN, L. (1981). The neon color effect in the Ehrenstein illusion. Perception, 10, 667-681.

Redies, C., Spillman, L., \& KunZ, K. (1984). Colored neon flanks and line gap enhancement. Vision Research, 24, 1301-1309.

Remole, A. (1970). Spatial characteristics of the border enhancement region in a flickering field. American Journal of Optometry, 47, 779786.

Remole, A., Ng, A. S. Y., Bathe, L. L., Padfield, P. D., Spafford M. M., \& SzYMKIW, M. A. (1985). Flicker haloes observed with subjective borders. Perception, 14, 31-40.

REYNOLDS, R. I. (1981). Perception of illusory contour as a function of processing time. Perception, 10, 107-115.

RICHARDSON, B. L. (1979). The nonequivalence of abrupt and diffuse illusory contours. Perception, 8, 589-593.

RoCK, I., \& ANSON, R. (1979). Illusory contours as the solution to a problem. Perception, 8, 665-681

Rowbury, C. R. (1982). A comment on Day and Jory's note on a second stage in the formation of illusory contours. Perception \& Psychophysics, 31, 395-396.

Sajda, P., \& Finkel, L. H. (1992a). Cortical mechanisms for surface segmentation. In F. Eeckman \& J. Bower (Eds.), Computation and neural systems 1992 (p. 10). Boston: Kluwer.

SAJDA, P., \& FINKEL, L. H. (1992b). Simulating biological vision with hybrid neural networks. Simulation, 59, 47-55.

SAJDA, P., \& FINKEL, L. H. (1995). Intermediate-level visual representations and the construction of surface perception. Journal of Cognitive Neuroscience, 7, 267-291

Salzman, B., \& Halpern, D. F. (1982). Subjective towers: Depth relationships in multilevel subjective contours. Perceptual \& Motor Skills, 55, 1247-1256.

Sambin, M. (1981). Figure anomale: La polarizzazione intrafigurale delle parti inducenti. Giornale Italiano di Psicologia, 8, 421-436.

SAMBIN, M. (1985). Figure anomale: La misura dell'ampiezza di una disomogeneità indotta. In W. Gerbino (Ed.), Conoscenza e struttura: Festschrift per Gaetano Kanizsa (pp. 437-452). Bologna: Il Mulino.

SAMBIN, M. (1987). A dynamic model of anomalous figures. In S. Petry \& G. E. Meyer (Eds.), The perception of illusory contours (pp. 131 142). New York: Springer-Verlag.

Schumann, F. (1900). Beiträge zur Analyse der Gesichtswahrnehmungen. Erste Abhandlung. Einige Beobachtungen über die Zusammenfassung von Gesichtseindruecken zu Einheiten. Zeitschrift für Psychologie und Physiologie der Sinnesorgane, 23, 1-32.

SchumanN, F. (1904). Einige Beobachtungen über die Zusammenfassung von Gesichtseindrücken zu Einheiten. Psychologische Studien, 1, 1-32.

SCRIVENER, S. (1983). Two for the price of one. Perception, 12, 769.

Shank, M. D., \& WALKER, J. T. (1989). Figure-ground organization in real and subjective contours: $A$ new ambiguous figure, some novel measures of ambiguity, and apparent distance across regions of figure and ground. Perception \& Psychophysics, 46, 127-138.

SHAPLEY, R., \& GoRDON, J. (1985). Nonlinearity in the perception of form. Perception \& Psychophysics, 37, 84-88.

SHAPLEY, R. \& GoRDON, J. (1987). The existence of interpolated illusory contours depends on contrast and spatial separation. In S. Petry \& G. E. Meyer (Eds.), The perception of illusory contours (pp. 109 115). New York: Springer-Verlag.

Shipley, T. F., \& Kellman, P. J. (1990). The role of discontinuities in the perception of subjective figures. Perception \& Psychophysics, 48 259-270.

Shipley, T. F., \& Kellman, P. J. (1992a). Perception of partly occluded objects and illusory figures: Evidence for an identity hypothesis. Jour nal of Experimental Psychology: Human Perception \& Performance, 18, 106-120.

Shipley, T. F., \& Kellman, P. J. (1992b). Strength of visual interpolation depends on the ratio of physically specified to total edge length. Perception \& Psychophysics, 52, 97-106.

ShIPLEY, T. F., \& KELlMAN, P. J. (1994). Spatiotemporal boundary formation: Boundary, form, and motion perception from transformations of surface elements. Journal of Experimental Psychology: General, 123, 3-20.

SIEGEL, S., \& PETRY, S. (1991). Evidence for independent processing of subjective contour brightness and sharpness. Perception, 20, 233-241.

Simmonds, M. B. (1974). Stereopsis and subjective contours. Perception \& Psychophysics, 15, 401-404.

SMith, A. T., \& Over, R. (1975). Tilt aftereffects with subjective contours. Nature, 257, 581-582. 
Smith, A. T., \& Over, R. (1976). Color-selective tilt aftereffects with subjective contours. Perception \& Psychophysics, 20, 305-308

Smith, A. T., \& Over, R. (1977). Orientation masking and the tilt illusion with subjective contours. Perception, 6, 441-447.

Smith, A. T., \& Over, R. (1979). Motion aftereffect with subjective contours. Perception \& Psychophysics, 25, 95-98.

Soriano, M., SPILlman, L. A., \& BACH, M. (1994). The abutting grating illusion. Manuscript submitted for publication.

SoubiteZ, M. C. (1982). Perceptual development of the Ehrenstein illusion in children [abstract]. Perception, 11, 33

Spillman, L. A., Fuld, K., \& Gerrits, H. J. M. (1976). Brightness contrast in the Ehrenstein illusion. Vision Research, 16, 713-719.

Spillman, L. A., Fuld, K., \& Neumeyer, C. (1984). Brightness matching, brightness cancellation, and increment threshold in the Ehrenstein illusion. Perception, 13, 513-520.

Stadler, M., \& Dieker, J. (1972). Untersuchungen zum Problem virtueller Konturen in der visuellen Wahrnehmung. Zeitschrift für Experimentelle und Angewandte Psychologie, 19, 325-350.

STAPPERS, P. J. (1989). Forms can be recognized from dynamic occlusion alone. Perceptual \& Motor Skills, 68, 243-251.

STEVENs, K. A. (1983). Evidence relating subjective contours and interpretations involving interposition. Perception, 12, 491-500.

TAKAHASHI, S. (1993). Microgenetic process of perception of subjective contour using "self-sufficient" inducing pattern. Perceptual \& Motor Skills, 77, 179-185.

TYLER, C. W. (1977). Is the illusory triangle physical or imaginary? Perception, 6, 603-604.

Tynan, P., \& Sekuler, R. (1978, May 30). Moving visual phantoms: A new contour completion effect. Science, 188, 951-952.

UlLMAN, S. (1976). Filling-in the gaps: The shape of subjective contours and a model for their generation. Biological Cybernetics, 25, 1-6.

VallorTigara, G. (1987). The hidden face of Kanizsa's triangle: Apparent movement of subjective figures in three-dimensional space. Perception, 16, 449-452.

VAllortigara, G., Bressan, P., \& Zanforlin, M. (1986). The Saturn illusion: A new stereokinetic effect. Vision Research, 26, 811-813.

vaN TUIJL, H. F. J. M. (1975). A new visual illusion: Neonlike color spreading and complementary color induction between subjective contours. Acta Psychologica, 39, 441-445.

van Tuisl, H. F. J. M., \& De Weert, C. M. M. (1979). Sensory conditions for the occurrence of the neon spreading illusion. Perception, $\mathbf{8}$, 211-215.

van TuiJl, H. F. J. M., \& LeeuwenberG, E. L. J. (1979). Neon color spreading and structural information measures. Perception \& Psychophysics, 25, 269-284

van TuiJL, H. F. J. M., \& LeEuWenberg, E. L. J. (1982). Peripheral and central determinants of subjective contour strength. In H. G. Geissler \& P. Petzold (Eds.), Psychophysical judgment and the process of perception (pp. 114-131). New York: North-Holland.

VARIN, D. (1971). Fenomeni di contrasto e diffusione cromatica nell'organizzazione spaziale del campo percettivo. Revista di Psicologia, 65, 101-128.

Vogels, R., \& Orban, G. A. (1987). Illusory contour orientation discrimination. Vision Research, 27, 453-467.

VON DER HeydT, R., \& PETERHANS, E. (1989a). Cortical contour mechanisms and geometrical illusions. In D. M. Lam \& C. D. Gilbert (Eds.), Neural mechanisms of visual perception (pp. 157-170). The Woodlands, TX: Portfolio Publishing. von der Heydt, R., \& Peterhans, E. (1989b). Mechanisms of contour perception in monkey visual cortex: I. Lines of pattern discontinuity. Journal of Neuroscience, 9, 1731-1748.

von der Heydt, R., Peterhans, E., \& Baumgartner, G. (1984, June 15). Illusory contours and cortical neuron responses. Science, 224, 1260-1262.

VON GRÜNAU, M. W. (1979). The involvement of illusory contours in stroboscopic motion. Perception \& Psychophysics, 25, 205-208.

Walker, J. T., \& Shank, M. D. (1987). The Bourdon illusion in subjective contours. Perception \& Psychophysics, 42, 15-24.

WALKER, J. T., \& SHANK, M. D. (1988a). Interactions between real and subjective contours in the Bourdon illusion. Perception \& Psychophysics, 43, 567-574.

WaLker, J. T., \& Shank, M. D. (1988b). Real and subjective lines and edges in the Bourdon illusion. Perception \& Psychophysics, 43, 475484.

Wallach, H., \& Slaughter, V. (1988). The role of memory in perceiving subjective contours. Perception \& Psychophysics, 43, 101-106.

WARE, C., \& KENNEDY, J. M. (1977). Illusory line linking solid rods. Perception, 6, 601-602.

Ware, C., \& Kennedy, J. M. (1978a). Perception of subjective lines, surfaces and volumes in 3-dimensional constructions. Leonardo, 11, 111-114.

WARE, C., \& KENNEDY, J. M. (1978b). The phenomenon of subjective contours. Leonardo, 11, 348

Warm, J. S., Dember, W. N., Padich, R. A., Beckner, J., \& Jones, S. (1987). The role of illumination level in the strength of subjective contours. In S. Petry \& G. E. Meyer (Eds.), The perception of illusory contours (pp. 176-182). New York: Springer-Verlag.

WASSERSTEin, J., ZaPpulla, R., Rosen, J., \& Gerstman, L. (1987). In search of closure: Subjective contour illusions, Gestalt completion tests, and implications. Brain \& Cognition, 6, 1-14.

WATANABE, T., \& OYAMA, T. (1988). Are illusory contours a cause or a consequence of apparent differences in brightness and depth in the Kanizsa square? Perception, 17, 513-521.

WATANABE, T., \& SATO, T. (1989). Effects of luminance contrast on color spreading and illusory contour in the neon color spreading effect. Perception \& Psychophysics, 45, 427-430.

WaTANABE, T., \& TAKEICHI, H. (1990). The relation between color spreading and illusory contours. Perception \& Psychophysics, 47, 457-467.

Weisstein, N., Maguire, W., \& Berbaum, K. (1977, December 2). A phantom-motion aftereffect. Science, 198, 955-958.

WENDEROTh, P., CRISS, G., \& VAN DER ZwAN, R. (1990). Determinants of subjective contour: Bourdon illusions and "unbending" effects. Perception \& Psychophysics, 48, 497-508.

WhitMore, C. L. G., LaWSON, R. B., \& Kozora, C. E. (1976). Subjective contours in stereoscopic space. Perception \& Psychophysics, 19, 211-213.

Zanforlin, M., \& Vallortigara, G. (1990). The magic wand: A new stereokinetic anomalous surface. Perception, 18, 410.

ZEKI, S. M., \& SHIPP, S. D. (1988). The functional logic of cortical connections. Nature, 335, 311-317.

ZuCKER, S. W., \& DAVIS, S. (1988). Points and endpoints: A size/spacing constraint for dot grouping. Perception, 17, 229-247.

(Manuscript received July 14, 1994; revision accepted for publication February 16, 1995.) 